\section{AIII02 59}

NAT'L INST OF STANDARDS \& TECH R.I.C.

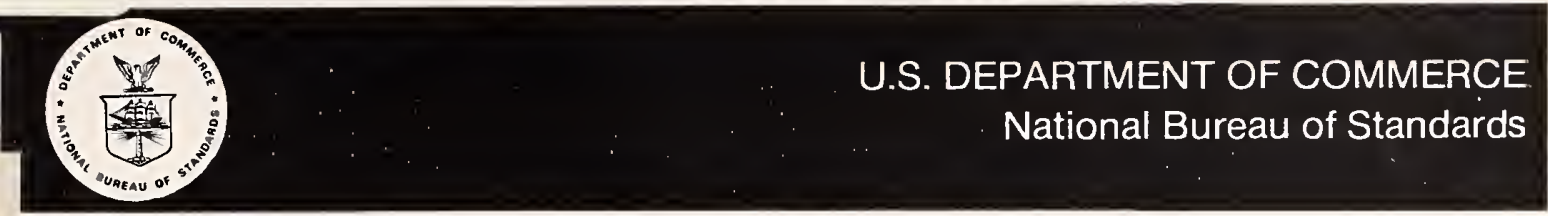

NBSIR 86-3385-1

\title{
Implementation Agreements for Open Systems Interconnection Protocols
}

NBS Workshop

for Implementors of Open Systems Interconnection

Revised July 24, 1986

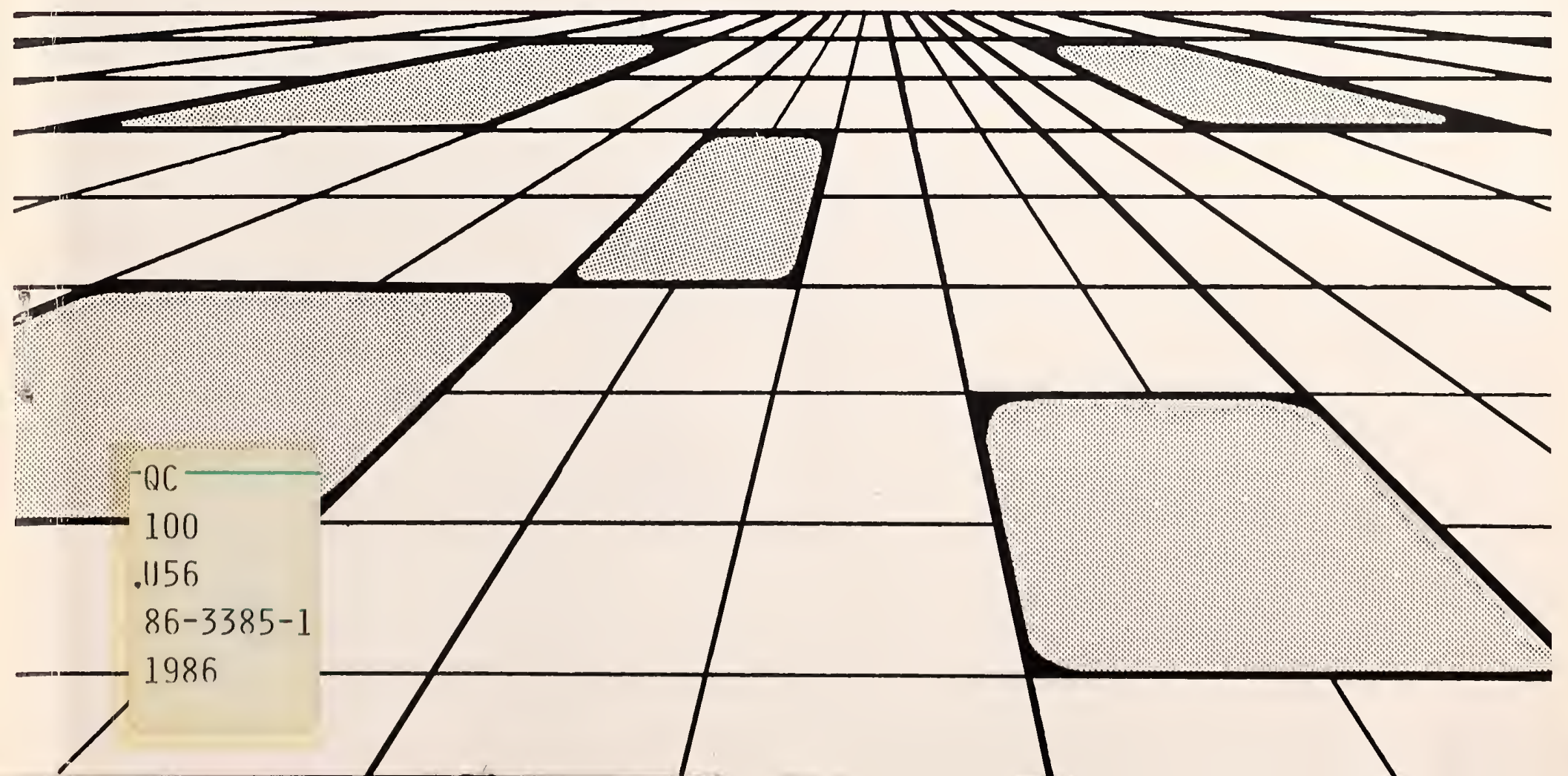


.

. 


\section{Implementation Agreements for Open Systems Interconnection Protocols}

NBS Workshop

for Implementors of

Open Systems Interconnection

Revised July 24, 1986 
" 
$08 / 13 / 86$

To Whom it May Concern:

This is to advise you that the manuscript, Implementation Agreements Among Implementors of OSI Protocols has been approved by WERB and assigned an NBSIR number 86-3385-1. If there are any questions concerning this note as the approval, please call WERB office,

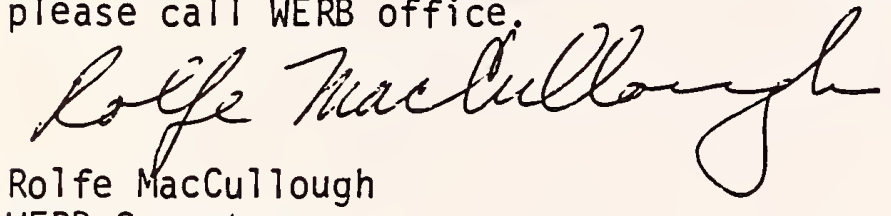

WERB Secretary

X2058 
List of Tables....................................... vi

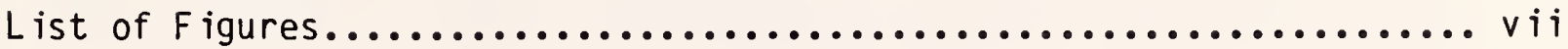

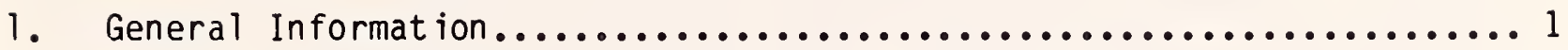

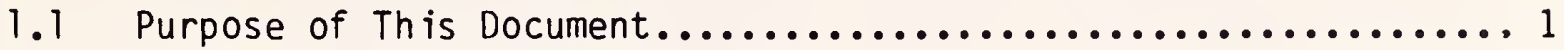

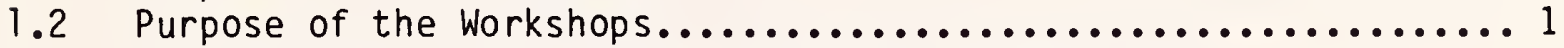

1.3 Relationship of Workshops to Outgrowing Events and

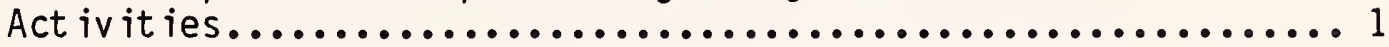

1.4 Relationsh ip of the Workshops to the NBS Laboratories..........2

1.5 Structure and Operation of the Workshops....................

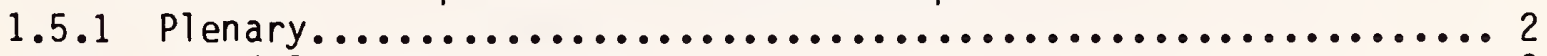

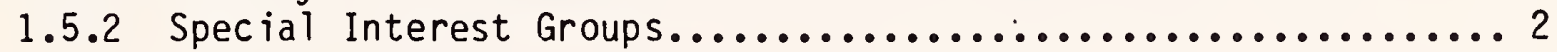

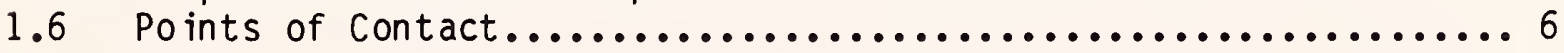

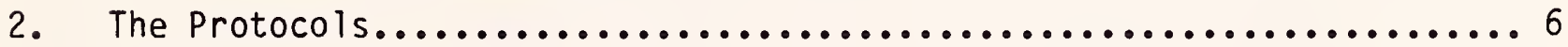

3. Local Area Networks................................... 6

3.1 IEEE 802.2 Logical Link Control ........................ 6

3.2 IEEE 802.3 CSMA/CD Access Method....................... 7

3.3 IEEE 802.4 Token Bus Access Method...................... 7

4. Wide Area Networks..................................... 8

4.1 CCITT Recommendation $x .25 \ldots \ldots \ldots \ldots \ldots \ldots \ldots \ldots \ldots \ldots \ldots \ldots$

5. Private Subnetworks.................................. 8

5.1 Private Subnetworks.............................. 8

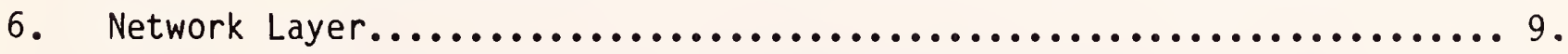

6.1 Connect ionless Network Service (CLNS) .................. 9

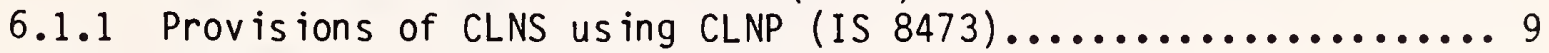

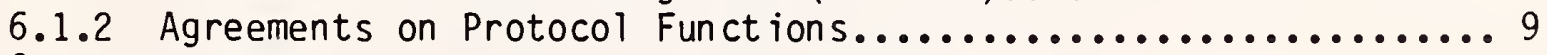

6.1 .3 Agreements on Optional Protocol Functions.................. 9

6.1.4 Network Dependent Convergence Sublayer Funct ion

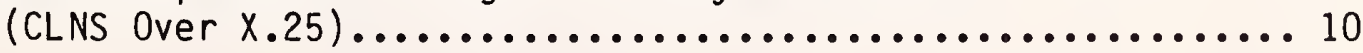

6.2 Connection Mode Network Service (CONS) ................... 10

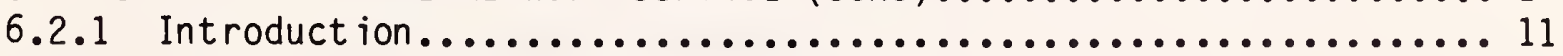

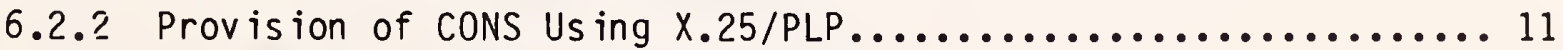

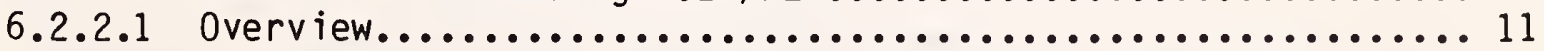

6.2 .2 .1 .1 Elements of the $X .25 / P L P$ for Support of the CONS....... 11

6.2.2.1.2 General Operation of the X.25/PLP-1984 for

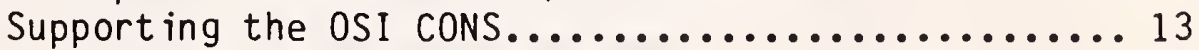

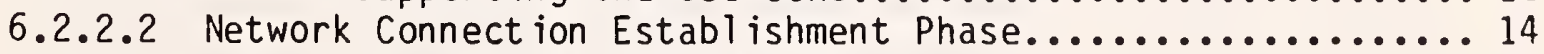

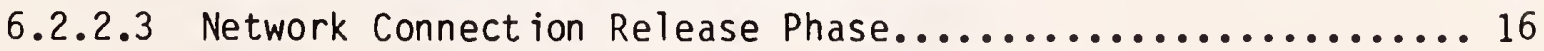

6.2.2.4 Data Transfer Phase -- Data Transfer Service.............. 16

6.2.2.5 Data Transfer Phase -- Rece ipt Confirmation Service........ 17

6.2.2.6 Data Transfer Phase -- Expedited Data Transfer

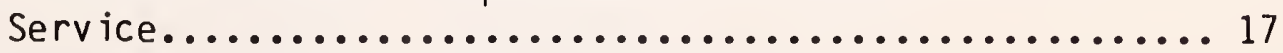

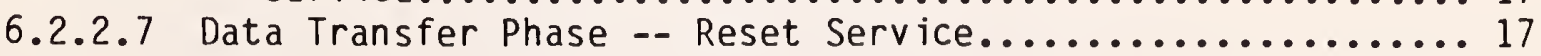


6.2.3 Requirements for Underlying Layer..................... 18

6.2.4 Cons ideration of OSI Transport Layer Protocol

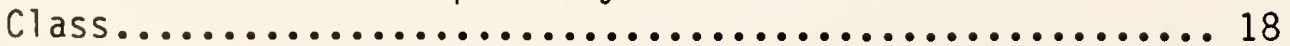

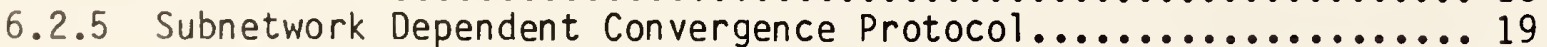

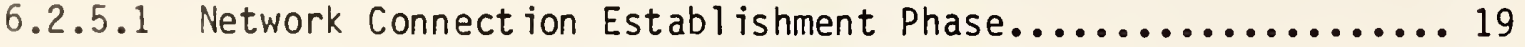

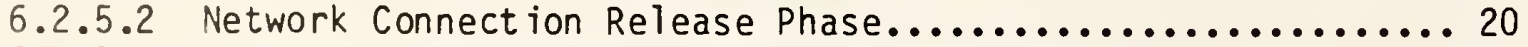

6.2.5.3 Data Transfer Phase - Data Transfer Service...............21

6.2.5.4 Data Transfer Phase - Rece ipt Confimation Service.........21

6.2.5.5 Data Transfer Phase - Expedited Data Transfer

Service..................................... 21

6.2 .5 .6 Data Transfer Phase - Reset Service................... 21

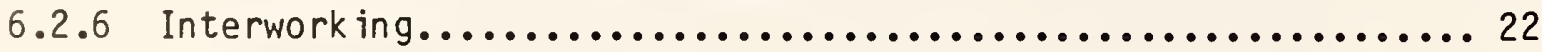

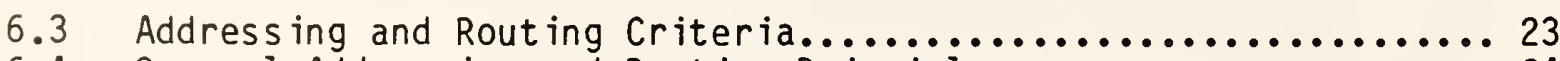

6.4 General Addressing and Rout ing.Principles.................. 24

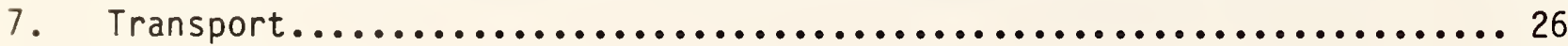

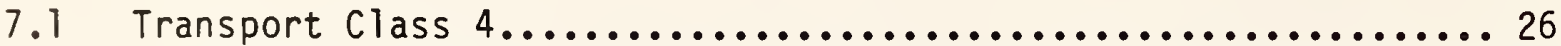

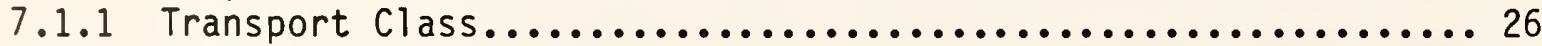

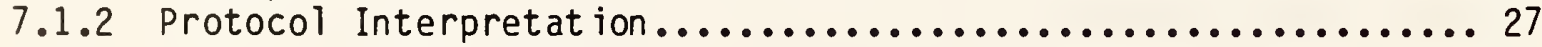

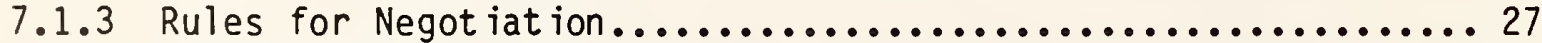

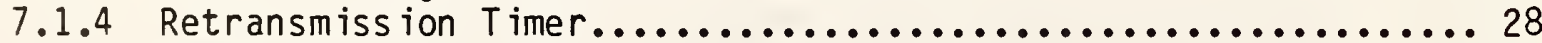

* 7.1 .5 Keep-Al ive Funct ion.................................. 29

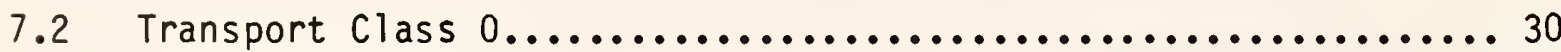

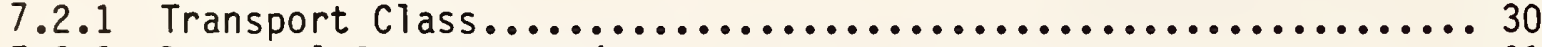

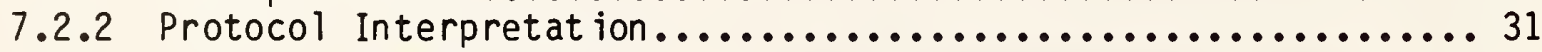

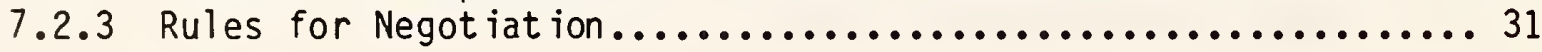

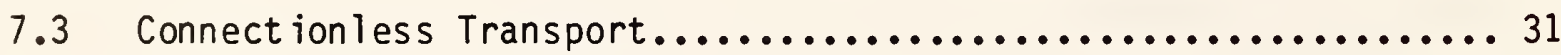

8. Session $\ldots \ldots \ldots \ldots \ldots \ldots \ldots \ldots \ldots \ldots \ldots \ldots \ldots \ldots \ldots \ldots \ldots \ldots \ldots \ldots \ldots \ldots \ldots$

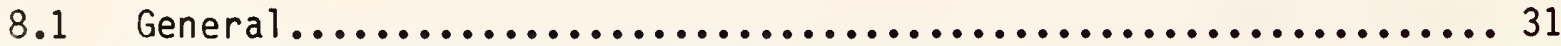

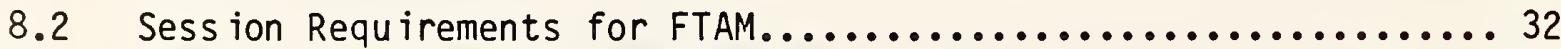

8.3 Sess ion Requirements for Message Handling................. 32

9. Service Access Points and Selectors......................... 33

* 9.1 Upper Layer Agreements................................. 33

9.2 Transport Class 4 Service Access Points or Selectors........... 33

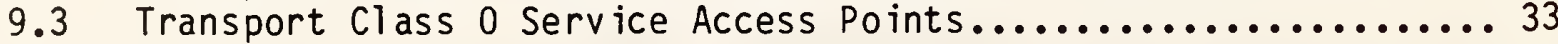

10. Iso File Transfer and Access Management Protocol................. 34

10.1 Phase 1 FTAM Implementation Specification................. 34

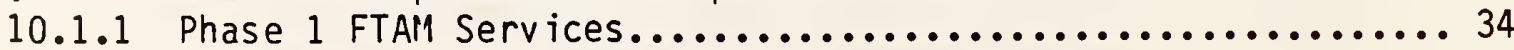

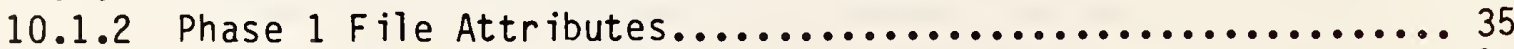

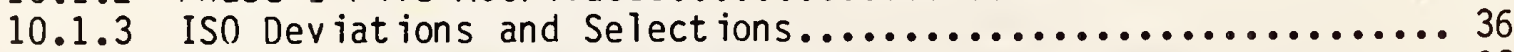

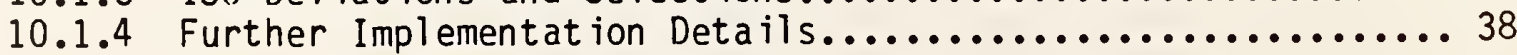

10.2 Phase 2 FTAM Implementation Spec if ication.................. 40

10.2 .1 Assumptions.................................. 40 


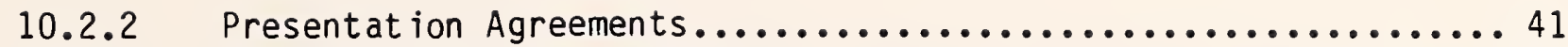

10.2.3 FTAM Service Type Agreements.......................... 42

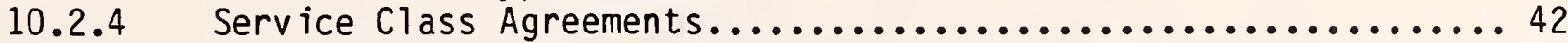

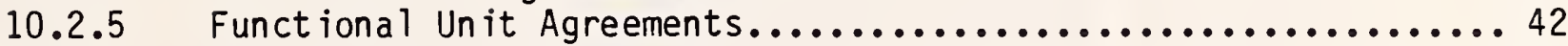

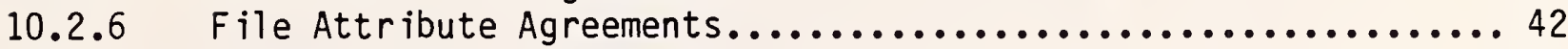

10.2.7 Document Type Agreements.............................. 43

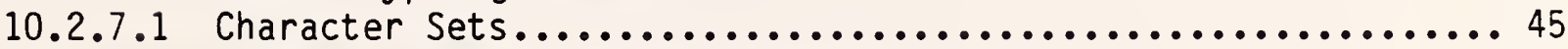

*10.2.7.2 Document Type Negot iation Rules....................... 46

*-10.2.7.3 Relationsh ip Between DUs, DEs and Document Types..........47

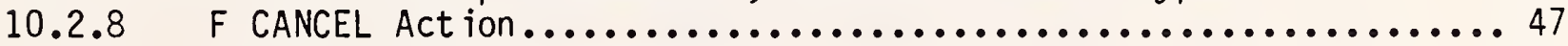

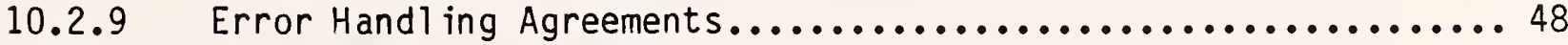

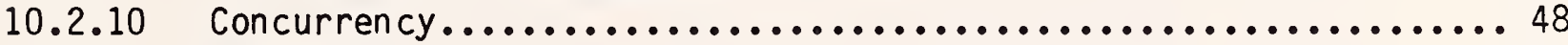

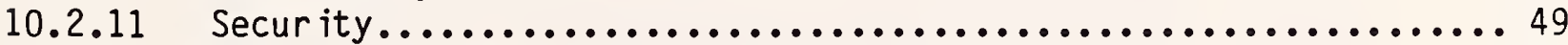

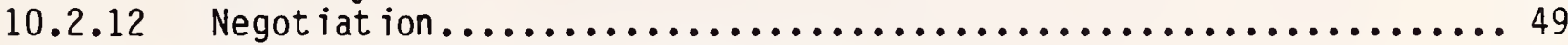

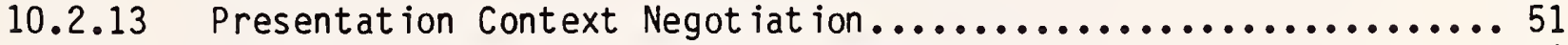

* 10.2.13.1 Steps of Presenta ion Context Negot iation................ 51

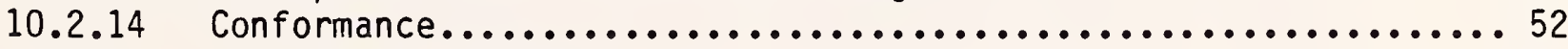

10.2 .15 Migration Strategy................................. 52

* 11. Iso Presentation Layer Protocol............................... 53

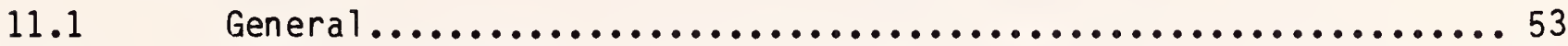

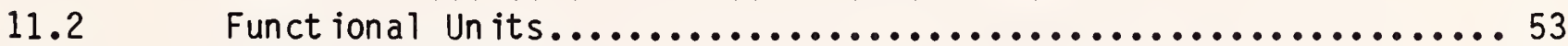

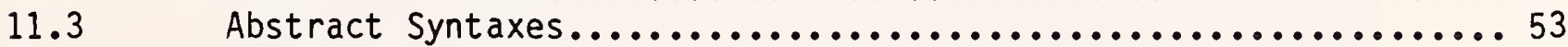

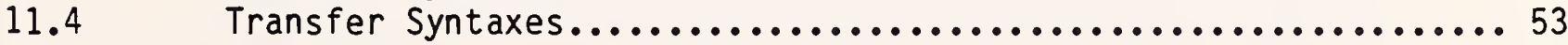

* 12. Common Application Service Elements Protocol........................ 54

$12.1 \quad$ General $\ldots \ldots \ldots \ldots \ldots \ldots \ldots \ldots \ldots \ldots \ldots \ldots \ldots \ldots \ldots \ldots \ldots \ldots \ldots \ldots \ldots \ldots \ldots$

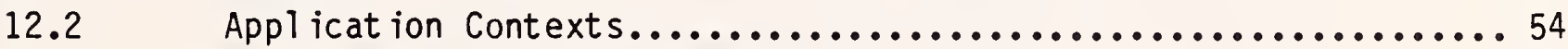

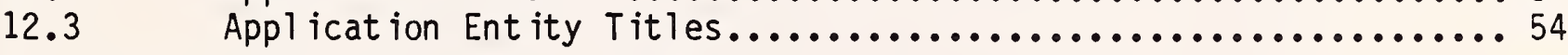

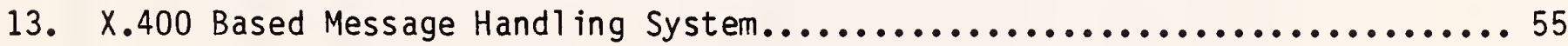

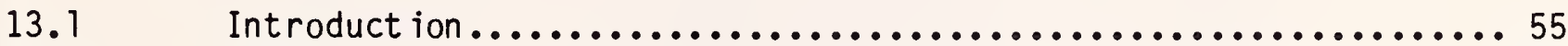

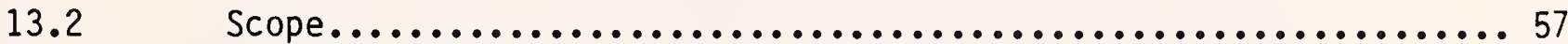

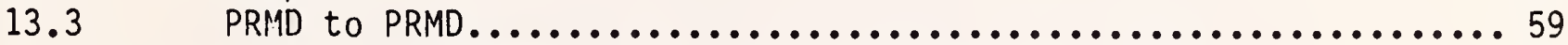

13.3.1 Service Elements and Optional User Facilities............6. 61

13.3.1.1 Class if ication of Support for Services................61

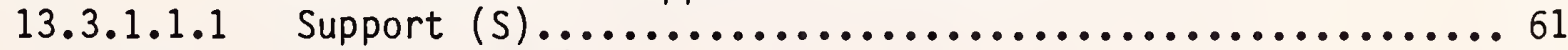

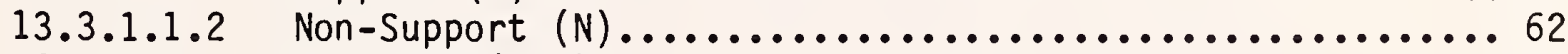

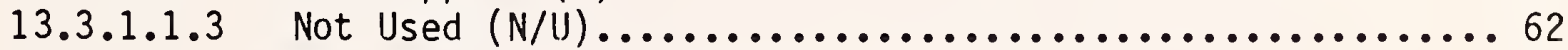

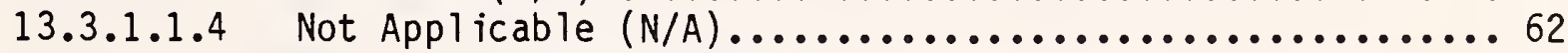

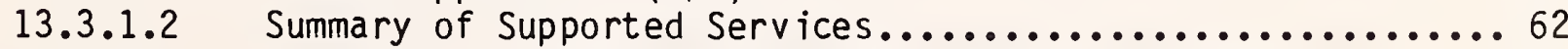

13.3.1.3 MT Service Elements and Opt ional User Facil it ies..........62

13.3.1.4 IPM Service Elements and Optional User Facil it ies.........64

$* 13.3 .2$

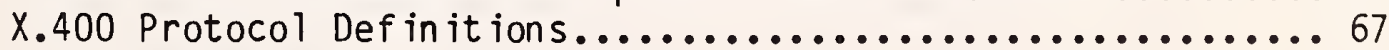

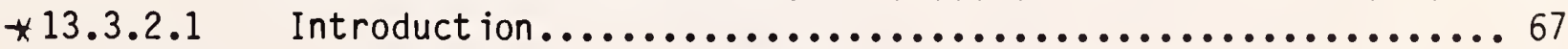

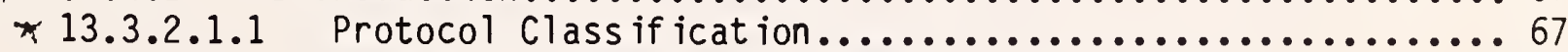

*13.3.2.1.2 General Statements on Pragmat ic Constraints..........66

\$13.3.2.1.3 MPDU Size.....................................68

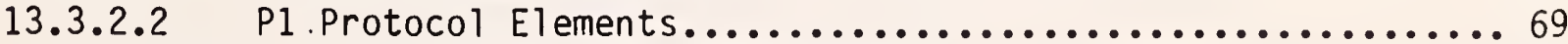

13.3.2.2.1 P1 Envelope Protocol Elements......................69

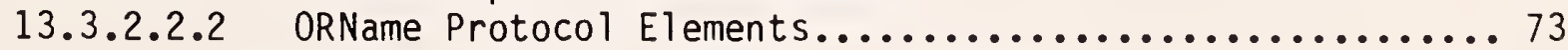

13.3.2.3 P2 Protocol Profile (Based on $[X .420]) \ldots \ldots \ldots \ldots \ldots \ldots \ldots . \ldots 75$

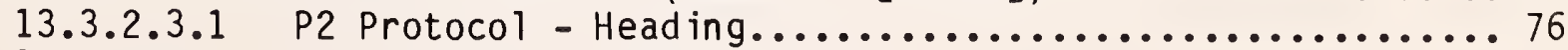

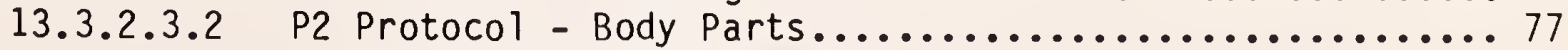

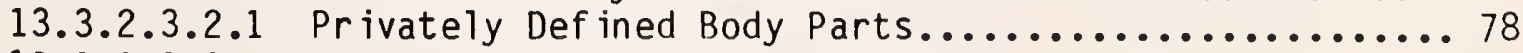

13.3.2.3.2.2 P2 Body Parts Protocol Elements................. 79 


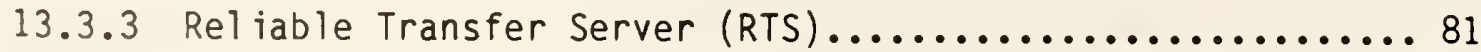

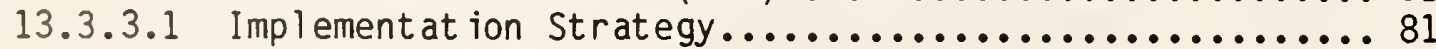

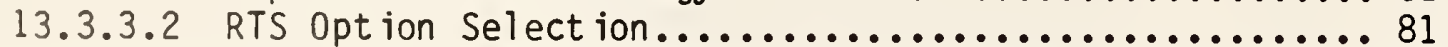

13.3.3.3 RTS Protocol Options and Clarifications.............. 82

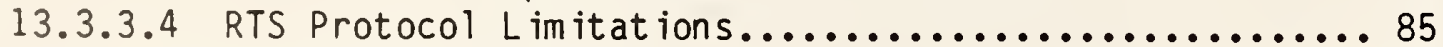

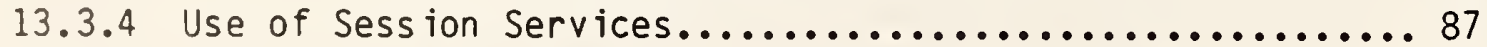

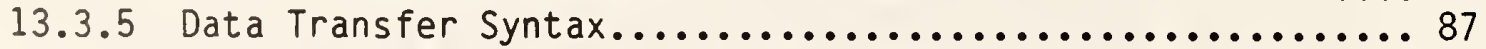

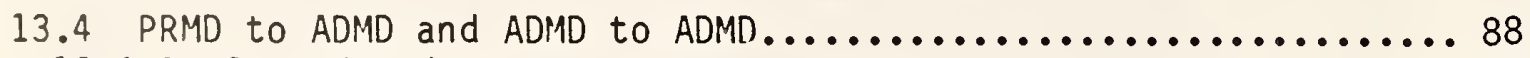

13.4 .1 Introduction................................. 88

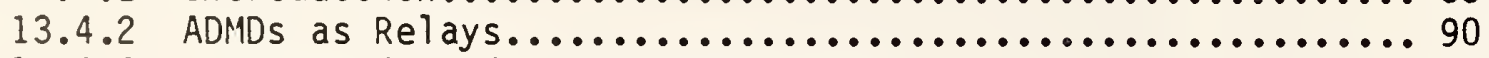

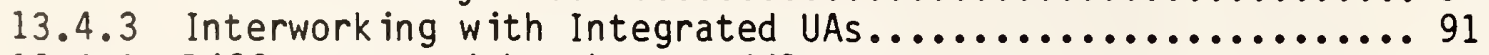

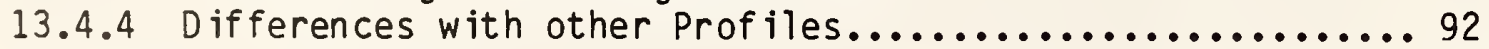

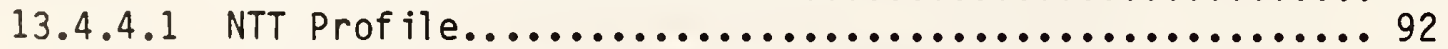

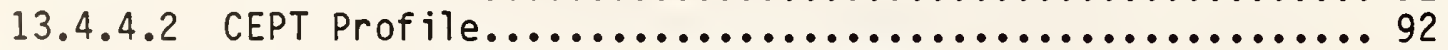

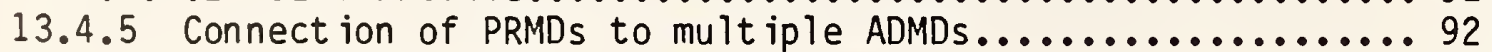

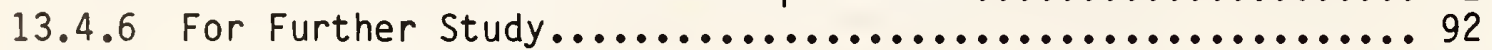

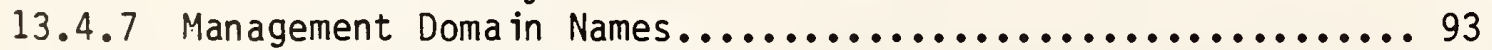

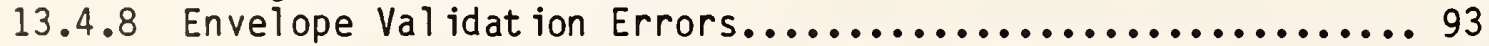

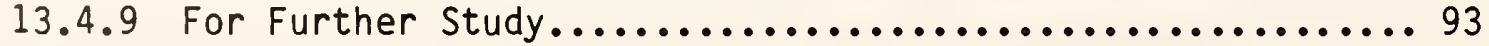

13.5 Error Reporting....................................... 94

13.5.1 MPDU Encoding..................................... 94

13.5 .2 Contents....................................... 94

13.5.3 Envelope....................................... 94

13.5.3.1 Pragmat ic Constraint Violations.................... 94

13.5.3.2 Protocol Violations................................ 94

13.5.3.3 $0 /$ R Names........................................ 95

13.5.3.4 Trace Informat ion................................. 95

13.5.3.5 Unsupported X.400 Protocol Elements................. 95

13.5.3.5.1 deferredDel ivery............................ 95

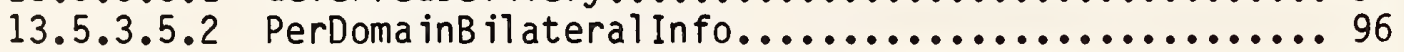

13.5.3.5.3 Expl ic itConvers ion......................... 96

13.5.3.5.4 alternateRec ip ientAl l owed................... 96

13.5.3.5.5 contentReturnRequest....................... 96

13.5.3.6 Unexpected Values for INTEGER Protocol Elements....... 96

13.5 .3 .6 .1 Priority................................ 96

13.5.3.6.2 Expl icitConvers ion........................ 96

13.5.3.6.3 ContentType............................... 97

13.5.3.7 Add it ional Service Elements.................... 97

13.5 .4 Reports....................................... 97

13.6 MHS Use of Directory Services $. . \ldots \ldots \ldots \ldots \ldots \ldots \ldots \ldots . \ldots 97$

13.7 Conformance.......................................... 98

13.7.1 Def in it ion of Conformance............................. 99

13.7.2 Conformance Requirements........................... 100

13.7.2.1 In it ial Conformance............................ 100

13.7.2.1.1 Services................................... 101

13.7.2.1.2 Protocol El ements............................ 101

* 14. Directory Services Protocols.............................. 102

* 15. Performance............................................ 102

+16 . Security........................................... 102 
References............................................... 103

Appendix A: Interpretation of Service Elements................ 108

Append ix B: Recommended Practices......................... 112

Appendix C: Rendition of IA5Text and T61String Characters......... 115

Append ix D: FTAM Document Types........................... 116

Addendum 1: Note on FTAM and X.400 Characters Sets................ 140

Reader Response Form..................................... 141 


\section{$\underline{\text { LIST OF TABLES }}$}

6.1 Packets and fields of the X.25/PLP used to support

the OSI CONS....................................... 12

6.2 CONS X.25/PLP mapp ing for the network connection establ ishment phase.............................................. 15

6.3 CONS: X.25/PLP mapping for the network connect ion release phase................................. 16

6.4 CONS: X.25/PLP mapping for the data transfer service....... 17

6.5 CONS: X.25/PLP mapping for the reset service............ 18

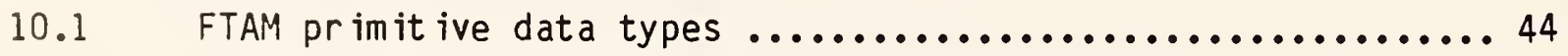

10.2 FTAM negotiation rules.............................. 49

13.3.1 Bas ic MT Service Elements..........................6 63

13.3.2 MT Optional User Facil it ies Provided to the UA-selectable on a Per-message Basis.......................................6 63

13.3.3 MT Optional User Facil ities Provided to the UA Agreed for a Contractual Period of Time............................6 64

13.3.4 Bas ic IPM Service Elements.......................... 64

13.3.5 IPM Optional Facil it ies Agreed for a Contractual Period of Time................................................. 65

13.3.6 IPM Optional User Facil it ies Selectable on a Per-Message Bas is .............................................66 66

13.3.7 P1 Protocol Elements..............................69

13.3.8 ORName Protocol Elements............................ 73

13.3.9 P2 Heading Protocol Elements.......................... 76

13.3.10 P2 BodyParts..................................... 79

13.3.11 Checkpoint and Window Size of IP...................... 84

13.3.12 RTS Protocol Elements.............................. 85 


\section{LIST OF FIGURES}

3.1 LSAP bit pattern................................... 7

6.1 Successful NC establ ishment............................ 20

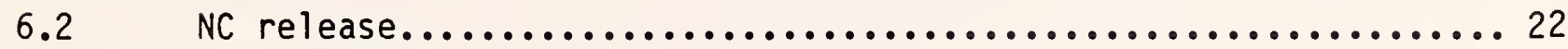

6.3 General ized interwork ing for OSI CONS................... 23

$6.4 \quad$ NSAP address format.......................................... 26

7.1 AK exchange on idle connection......................... 30

10.1 FTAM primitive data types............................ 44

13.1.1 The layered structure of this implementat ion agreement......... 56

13.2.1 This implementation agreement applies to the interfaces between:

A) PRMD and PRMD; B) PRMD and ADMD; and C) ADMD and ADMD...... 58

13.3.1 Interconnect ion of private doma ins.....................6 60

13.4.1 An ADMD may (b) or may not (a) serve as a relay..............89 
1. GENERAL INFORMATION

\subsection{PURPOSE OF THIS DOCUMENT}

This document records current agreements on implementation details of OSI protocols among the organizations participating in the NBS/OSI Workshop Series for Implementors of OSI Protocols. These decisions are documented to facilitate organizations in their understanding of the status of agreements. This is a standing document that is intended to be updated after each workshop (about every 2-1/2 months).

\subsection{PURPOSE OF THE WORKSHOPS}

In February, 1983 NBS organized the above named workshops to bring together future users and potential suppliers of OSI protocols. The workshop accepts as input the specifications of emerging standards for protocols and produces as output agreements on the implementation and testing particulars of these protocols. This process is expected to expedite the development of OSI protocols and promote interoperability of independently manufactured data communications equipment.

\subsection{RELATIONSHIPS OF WORKSHOPS TO OUTGROWING EVENTS AND ACTIVITIES}

The workshops are held for those organizations expressing an interest in implementing OSI protocols. However, there is no corporate commitment to implementations associated with workshop participation. Other events stem from the workshops to which commitments are attached. Sixteen organizations did make formal commitments to implement and demonstrate some of the protocols at the 1984 National Computer Conference. Commitments were made by General Motors, Boeing Computer Services, and 21 vendors to demonstrate, at AUTOFACT 1985, a set of OSI protocols known as the Manufacturing Automation Protocols or MAP specification. This event was an outgrowth of the workshops in that the implementation decisions reached in the workshops were used for the AUTOFACT demonstration. However, the AUTOFACT demonstration was planned and carried out by GM, BCS, and suppliers they selected. This event had no further affiliation with the workshops.

A different activity, initiated by NBS, is the OSINET, which is to be a long standing, globally distributed network that is put in place for purposes such as test methods development and testing of prototype implementations. Presently, 25 organizations have committed to participate. As with the AUTOFACT demonstration, protocols used on OSINET are those agreed to in the workshop. Also, as with the AUTOFACT demonstration, the OSINET has no other affiliation with the workshops. Inlike the AUTOFACT demonstration, however, OSINET participation is open to any organization committing to the OSINET agreements. 


\subsection{RELATIONSHIP OF THE WORKSHOPS TO THE NBS LABORATORIES}

As resources permit, NBS, with voluntary assistance from industry, develops formal protocol specifications, reference implementations, tests and test systems for the protocols agreed to in the workshops. This is work made available to the industry volunteers and to others making valid commitments to organized events and activities such as NCC, AUTOFACT, and OSINET. As soon as this work can be adequately documented it is placed in the public domain through submission to the National Technical Information Service. Any organization may then obtain the work at nominal charge.

The NBS laboratories bear no other relationship to the workshops.

\subsection{STRUCTURE AND OPERATION OF THE WORKSHOPS}

\subsubsection{Plenary}

The main body of the workshops is a plenary assembly. Any organization may participate. Representation is international. NBS prefers for the business of workshops to be conducted informally, since there are no corresponding formal commitments within the workshops by participants to implement the decisions reached. The guidelines we follow are: 1) one vote per company or independent division, 2) only companies that regularly attend should vote, 3) only companies that plan to sell or buy a protocol should vote on its implementation decisions, 4) only companies knowledgeable of the issues should vote, and 5) no proxy votes are admissible.

\subsubsection{Special Interest Groups}

Within the workshops there are Special Interest Groups (SIGs). The SIGs receive their instructions for their technical program of work from the plenary. The SIGs meet independently, usually during the three day workshop. As technical work is completed by a SIG, it is presented to the plenary for disposition. Companies participating in a SIG are expected to participate in the plenary. Voting rules for SIGS are the same as voting rules for the plenary.

Special Interest Groups sometimes correspond with organizations performing related work, such as ANSI committees. Such correspondence should be sent through the plenary to the parent committee, such as ANSC X3T5 or ANSC X3S3. When SIG meetings take place between workshops, the correspondence from these meetings should be addressed directly to the parent committee and copied to the workshop plenary.

Following are procedures for cooperative work among special interest groups.

a) Any SIG (SIG 1) or individual having issues to discuss with or requirements of another SIG (SIG 2) should bring the matter to the attention of the chairperson of that SIG (SIG 2). 
b) The SIG 2 chairperson should bring the matter before SIG 2 for action.

c) SIG 2 should respond to the concerns or needs of SIG 1 or the individual in a timely manner.

d) If the matter cannot be sat isfactorily resolved or if the request is outs ide the charter assigned to SIG 1, then it should be brought before the plenary.

e) SIGs are expected to complete work in a timely manner and bring the results before the plenary for disposition. However, the plenary may elect to act on any issue with in the scope of the workshop at any time.

Following are the current charters of the the six Special Interest Groups. FTAM SIG

Develop phase 2 product-level specifications for FTAM as requested by the Corporation for Open Systems.

Future new work items will be defined in a phase 3 specification. It will contain only extensions of phase 2 FTAM. It is a goal that phase 3 will be backward compatible with phase 2 FTAM. The set of future work items 1 isted below may be changed by the plenary if the work is more appropriate for other SIGs.

High priority work items:

- Develop implementation specifications for ISO CASE and ISO Presentation protocols

- Clean up section 10 of this document

- Specify Reliable File Service

- Specify Recovery and Restart Data Transfer functional units in the user correctable file service

- Specify concurrency control parameter.

Low priority work items:

- Add new document types/constraint sets

- Define subset of author ization requirements

- Specify Presentation Context Management functional un it. 
Develop product-level specifications for Message Handling Systems us ing the CCITT X.400 Recommendations as requested by the Corporation for Open Systems.

Develop abstract tests for $X .400$, as requested by the ad hoc rapporteur for this study question in CCITT. This work is to be submitted by the plenary (after its approval) to The U.S. Department of State as a proposed U.S. contribution to CCITT Study Group VII.

\section{Lower Layer SIG}

The Lower Layer SIG will study OSI layers 1-4 and produce recommendations for implementations to support the projects undertaken by the workshops and the work of the other SIGs. Both connectionless and connectionor iented modes of operation will be studied. The SIG will accept direction from the plenary for work undertaken and the priority which it is assigned.

The objectives of the Lower Layer SIG are:

- Study OSI layers 1-4 as directed by the plenary,

- Produce and mainta in recommendations for implementation of these layers, and

- Where necessary, provide input to the relevant standards bodies concerning layers $1-4$, in the proper manner.

- Begin work on the implementation specification of the ISO Network Layer Rout ing Exchange Protocol prior to the ISO draft achieving DIS status.

\section{Performance SIG}

The plenary will provide the following inputs to the OSI Performance SIG:

o the set of applications for which the performance of OSI protocols is of particular concern,

o the requirements for each application including:

- performance targets

- network topology

- background network loads

- application traffic characteristics, and

- this document, "Implementation Agreements Among Implementors of OSI Protocols". 
The objectives of the OSI Performance SIG are to:

o determine whether the OSI protocols are able to meet these performance requirements,

- report these determinations to the plenary, and

o where appropriate, provide input to the voluntary stanards bodies concerning changes to existing standards and the requirements for new ones, in the appropriate form.

\section{OSI Security Architecture SIG}

GOAL: To develop an overall OSI Security Architecture which is consistent with the OSI and which economically satisfies the primary security needs of both the commercial and Government sectors.

APPROACH: To define a security architecture encompassing the security addenda presently being specified at certain OSI layers, the required cryptographic algorithms and related key management functions, and the security management functions which must be performed between the layers and the peer entitities defined in the OSI architecture.

\section{Directory Services SIG}

The DS SIG shall develop implementation specifications, at the request of The U.S. Navy, for directory user agent to directory systems agent and for directory systems agent to directory systems agent. These specifications shall be based upon the current work of CCITT and ISO in this area. 
1.6 POINTS OF CONTACT

OSI Workshop - General

John Heafner, NBS, 301/921-3537

OSI Workshop - Registration

Mary Lou Fahey, NBS, 301/921-3516

$\mathrm{K}$ im Brink, NBS, 301/921-3537

FTAM SIG

X.400 SIG

Lower Layers SIG

Performance SIG

Secur ity SIG

DS SIG

MAP

TOP

OSINET

Steering Comm ittee

Technical Committee

Rick Peterson, GM, 313/492-6705

John Stidd, Xerox, 415/496-6527

Kevin Miles, DEC, +44-734-868711

Mary Jane Stroh1, CDS, 617/460-0808

Denny Branstad, NBS, 301/921-3427

J. J. Cinecoe, WANG, 617/967-5514

Gary Workman, GM, 313/575-0632

Laurie Bride, BCS, 206/763-5719

Bob Blanc, NBS, 301/921-3817

Andy Poupart, Tandem, 408/725-6480

SME (MAP/TOP Sponsorship)

Mark Shaw, 313/271-1500

Paul Borawski, 313/271-1500

\section{THE PROTOCOLS}

The selected protocols for which implementation agreements have been made and are being developed are:

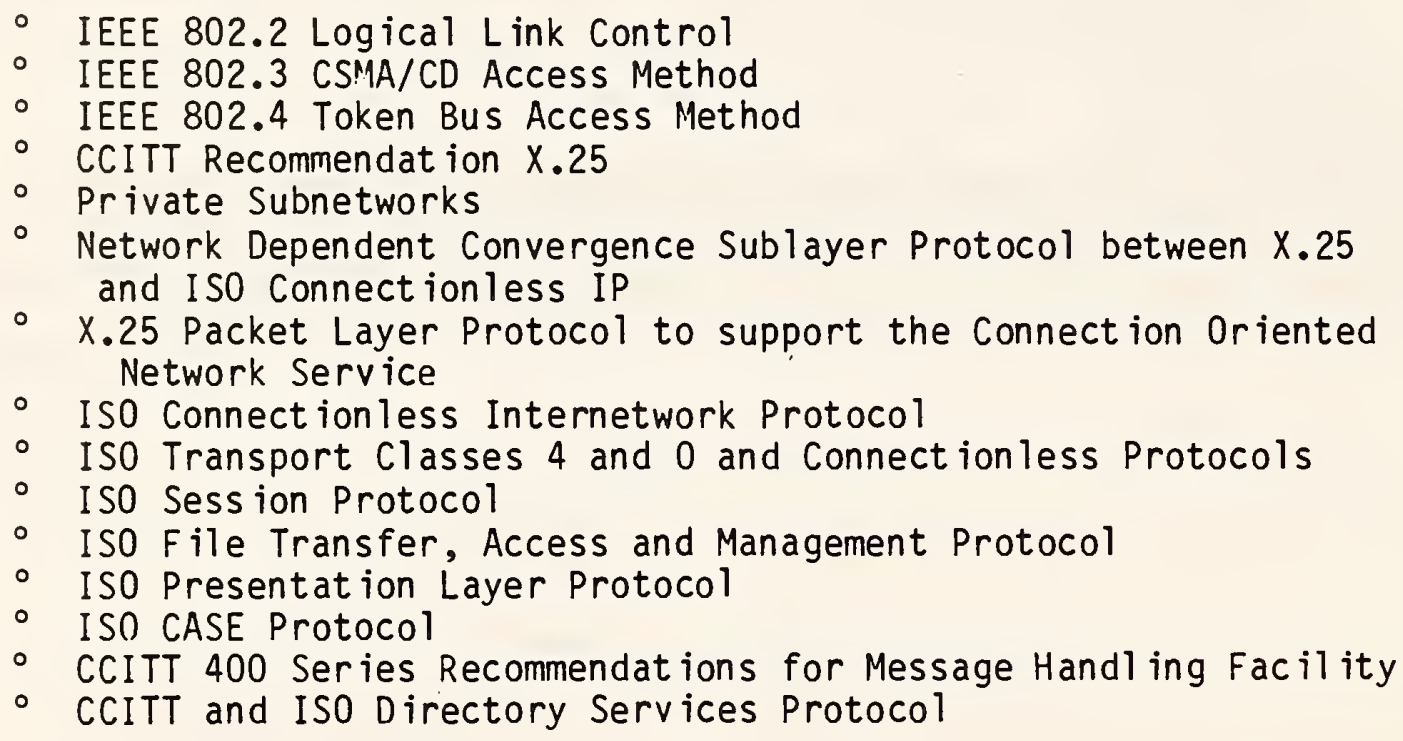

Reference documents and sources for obtaining them are given under REFERENCES.

\section{LOCAL AREA NETWORKS}

3.1 IEEE 802.2 LOGICAL LINK CONTROL

The following decisions have been reached with respect to th is protocol. 
1. Link Service Access Point (LSAP)

The IEEE 802 committee has assigned the code below to address systems using ISO IS 8473 connectionless network protocols. Note that bit zero is transmitted first.

The most significant bit is bit 7 , thus this bit pattern represents hexadecimal FE.

\begin{tabular}{|l|l|l|l|l|l|l|l|}
\hline 0 & 1 & 2 & 3 & 4 & 5 & 6 & 7 \\
\hline 0 & 1 & 1 & 1 & 1 & 1 & 1 & 1 \\
\hline
\end{tabular}

Fig. 3.1 LSAP bit pattern

2. Type and $\mathrm{Cl}$ ass

Only the connectionless type 1, class 1 IEEE 802 link service will be used.

3. Exchange Identification and Test

The Exchange Identification (XID) and Test (TEST) will not be sent. If one is received it will be discarded.

3.2 IEEE 802.3 CSMA/CD ACCESS METHOD

The following decision has been reached with respect to this protocol.

1. Addressing

The 48 bit addressing will be used with the 10 megabit/second baseband coaxial cable specification,

3.3 IEEE 802.4 TOKEN BUS ACCESS METHOD

The workshop participants agreed to inclusion of token bus. The following options were agreed to with respect to Draft $F$. An asterisk means that the option has been approved. The absence of an asterisk means that the option has not been approved.

1. Repeaters

2. Medium

Active Regenerative

Single Cable Coax

Dual Cable Coax

3. Trunk Cable

RG-6

RG-11

Semi-rigid

Other 75 ohm cables

4. Trunk Connection Unit

75 ohm tee connector

75 ohm nondirectional passive

impedence-matching tap 
75 ohm directional passive impedencematching tap

5. Transmit Carrier Frequency

RF

Baseband

6. Modulation

Phase Continuous FSK

Phase Coherent FSK

7. Encoding

AM/PSK

Manchester

Duobinary

8. Data Rate

$1 \mathrm{Mb}$

$5 \mathrm{Mb}$

$10 \mathrm{Mb}$

9. Addressing

2 octet

6 octet

10. Connector at Station

50 ohm Male BNC Series

75 ohm Female $F$ Series

11. Priority (4 levels)

12. Group Addressing

13. Station Management

14. Broadband Channel Assignments

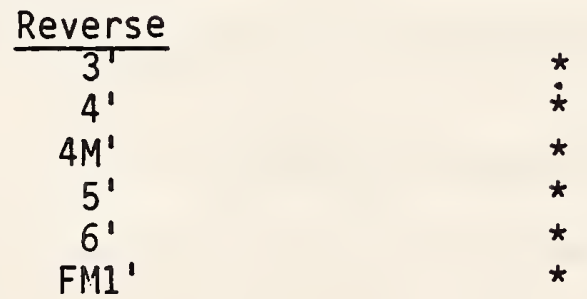

4. WIDE AREA NETWORKS

4.1 CCITT RECOMMENDATION X.25

When providing CONS, it has been agreed to use $x .25$ as the standard wide area network protocol. Elements of X.25 are explained in section 6.2.2.

\section{PRIVATE SUBNETWORKS}

\subsection{PRIVATE SUBNETWORKS}

The architectures agreed upon allow the use of private subnetworks in addition to private $X .25$ subnetworks. No particular private subnetwork has been discussed. 


\section{NETWORK LAYER}

\subsection{CONNECTIONLESS NETWORK SERVICE (CLNS)}

\subsubsection{Provisions of CLNS us ing CLNP (IS 8473)}

The following decisions have been reached with respect to this protocol.

1. The "Protocol for providing the connection-less service," ISO IS 8473 , will be used to provide the CLNS.

2. The full conformance protocol will be used for concatenated networking. The inactive subset may be used when communicating on a single subnetwork. ${ }^{+}$The inact ive subset is really the absence of an IP and is so indicated by an IP header of one octet of zeros. The nonsegmenting subset will not be supported.

\subsubsection{Agreements on Protocol Functions}

1. For purposes of demonstration the value to be used to bound the maximum lifetime of the internetwork protocol data un it is three times the network span. The span of the network is considered to be the number of intermediate systems between source and destination plus the destination end system.

2. For purposes of testing, intermediate and end systems will log the following conditions:

- Discarded protocol data units,

- Error protocol data units (recorded by system generating the error PDU), and

- Detection of protocol data un its containing unsupported type 3 options.

\subsubsection{Agreements on Opt ional Protocol Functions}

1. The security parameter will not be used.

2. Intermediate systems should recognize and support both complete and partial source routing.

Although end systems may implement source routing (of either type), any requirement for end system source routing is deferred for future study, since support for it may be related to layer management protocols.

\footnotetext{
t When using the inactive subset, the NSAP Selector must be absent, no segmenting is required, and the AFI and subnetwork number must be the same, i.e., denoting a single subnetwork attachment.
} 
3. Partial source routing will be supported by intermediate systems. The destination should $\log$ the route, if possible.

4. The ISO specification will be followed with respect to quality of service.

5. For purposes of testing, checksums will be turned on and used. In operation this is a local decision.

6.1.4 Network Dependent Convergence Sublayer Function (CLNS Over X.25)

A network dependent convergence sublayer protocol operating between CCITT Recommendation $\times .25$ and the ISO Connectionless IP has been agreed to. It shall adhere to the following.

1. Follow ISO 8473 DADI (N3601) Working Paper for the SNDCF framework.

2. Only initiate one SVC for all outbound PDUs to any other given intermediate system or PDN end system. Note: this results in at most two SVCs between any pair of systems.

3. Open a connection upon demand.

4. Disconnect SVCS by administrative request and a finite timeout timer.

5. Use the default throughput class from APS.

6. Do not use D-bit or Q-bit.

7. Negotiate window size according to ISO IS 8208. A default of window size two must be supported.

8. Negotiate packet size according to ISO IS 8208. A default of 128 octets/packet must be supported.

9. The SNDCF will "advertise" support for SNSDUs up to $1 \mathrm{~K}$ via the $\mathrm{X} .25 \mathrm{M}$ bit facility.

10. No statistics gathering is required beyond that which the CLNS may gather for the X.25 packet layer or for SNDCF entities.

6.2 CONNECTION MODE NETWORK SERVICE (CONS)

There is interest among a limited set of participants in implementation of the connection mode network service. This section records the agreement of the workshop on the provision of a connection mode network service. 


\subsubsection{Introduct ion}

The $X .25$ Packet Level Protocol (PLP) is to be used to provide of the OSI Connect ion-mode Network Service (CONS). This proposal is independent of lower and higher layer protocol considerations, which are also discussed briefly herein.

When providing the CONS, the following shall apply:

a. the def in ition of the CONS is as specified in ISO 8348 ("Network Service Def in it ion");

b. the mapping of the elements of the CONS to the elements of the X.25/PLP is as specified in ISO 8878 ("Use of X.25 to Provide the OSI Connect ion-mode Network Service"); and

c. the general procedures and formats of the X.25/PLP are as specified in ISO 8208 ("X.25 Packet Level Protocol for Data Terminal Equipment"); it should be noted that this standard provides for the use of the X.25/PLP in environments in addition to an X.25 PSPDN, such as po int-to-po int topologies as well as LANs. (The details of how to use the X.25/PLP in LANs are given in ISO 8881, "In format ion Process ing Systems - Data Commun icat ions Use of The X.25 Packet Level Protocol in Local Area Networks.")

\subsubsection{Provision of CONS Using X.25/PLP}

The provision of the CONS using the X.25/PLP is as described in (b) above. This section provides a brief description taken directly from ISO 8878; for more detail, see ISO 8878.

It should also be noted that the X.25/PLP-1984 is capable of supporting the full CONS, including qual ity-of-service (QOS) aspects. The X.25/PLP1984 shall be used in LANS and in packet-switched networks allowing the use of the elements of the X.25/PLP-1984 needed to support the CONS. In other packet-switched-network environments, a Subnetwork Dependent Convergence Protocol (SNDCP) shall be used in conjunction with the $X .25$ / PLP-1980 (see sect ion 6.2.5).

\subsubsection{Overview}

\subsection{Elements of the X.25/PLP for Support of the CONS}

The table below 1 ists the packets and associated fields used when support ing the OSI CONS. 
Table 6.1

Packets and fields of the X.25/PLP

used to support the OSI CONS

\begin{tabular}{|c|c|}
\hline PACKET TYPES 1 & FIELDS 2 \\
\hline $\begin{array}{l}\text { CALL REQUEST } \\
\text { INCOMING CALL } \\
\text { CALL ACCEPTED } \\
\text { CALL CONNECTED }\end{array}$ & $\begin{array}{l}\text { Facil ity Field, Call and } \\
\text { Called User Data Field }\end{array}$ \\
\hline $\begin{array}{ll}\text { CLEAR } & \text { REQUEST } \\
\text { CLEAR } & \text { INDICATION }\end{array}$ & $\begin{array}{l}\text { Clearing Cause Code Field, } \\
\text { Diagnostic Code Field, } \\
\text { Facil ity Field, Clear User } \\
\text { Data Field }\end{array}$ \\
\hline DATA & M-B it, User Data Field \\
\hline $\begin{array}{ll}\text { RESET } & \text { REQUEST } \\
\text { RESET INDICATION }\end{array}$ & $\begin{array}{l}\text { Resetting Cause Code } \\
\text { F ield, Diagnostic Code } \\
\text { F ield }\end{array}$ \\
\hline RESTART INDICATION & $\begin{array}{l}\text { Restarting Cause Code } \\
\text { Field, } \\
\text { Diagnost ic Code Field }\end{array}$ \\
\hline
\end{tabular}

Notes:

1. The packets shown in the table are used in support of the primitives of the OSI CONS. Other packets not shown in the table (i.e., CLEAR CONFIRMATION, RESET CONFIRMATION, and RESTART CONFIRMATION packets) are essential to the use of the packets shown. Yet other packets (i.e., RESTART. REQUEST, DIAGNOSTIC, REGISTRATION REQUEST, AND REGISTRATION CONFIRMATION packets) have no relationship to the provis ion of the OSI CONS.

In 1 ine with these agreements, the INTERRRUPT, INTERRUPT CONFIRMATION, RECEIVE READY, RECEIVE NOT READY, AND REJECT packets are not needed in support of the OSI CONS because the corresponding aspects of the OSI CONS are not to be used (i.e., the Expedited Data Transfer Service and the Rece ipt Confirmation Service are not used). However, the RECEIVE READY and RECEIVE NOT READY packets are needed for the proper operation of the $X .25 / P L P$. 
2. The information in the fields shown in the table have a direct relat ionship to the parameters associated with the primitives of the OSI CONS. Other fields not shown in the table (e.g., the Logical Channel Identifier, the Packet Type Ident if ier, the Address Length Fields and the Facility Length Field) are essential to the use of the appropriate packets.

In addition, it is also necessary for the following optional user facil ities and CCITT-Specified DTE Facil ities to be used and/or agreed to:

a. optional user facilities --

- Fast Select (facility used)

- Fast Select Acceptance (facil ity agreed to, if operat ing in a packet-switched network environment)

- Throughput Class Negot iation (facil ity agreed to and used), and

- Trans it Delay Selection And Indication (facility used);

Note: When operating in a DTE-to-DTE environment without an interven ing packetswitched network, the use of the Fast Select Facility Must also be agreed to by the two DTEs. In addition, the Fast Select Acceptance Facil ity does not apply.

b. CCITT-Specified DTE facilities--

- Called Address Extens ion (facility used),

- Call ing Address Extension (facil ity used),

- End-to-End Transit Delay Negotiation (facility used),

- Expedited Data Negotiation (facil ity used), and

o Min imum Throughput Class Negotiation (facil ity used).

Elements of the X.25/PLP not needed in support of the CONS are:

a. Q-bit

b. Permanent Virtual Circuits (PVCS);

c. Diagnostic packets; and

d. optional user facil it ies other than those 1 isted above.

Elements of the X.25/PLP not used with in the scope of these agreements are:

a. D-bit and

b. INTERRRUPT packets

6.2.2.1.2 General Operation of the X.25/PLP-1984 for Support ing the OSI CONS

The $X .25 / P L P$ can be used to provide the OSI CONS in an end system connected to a public or private $X .25$ packet-switched network environment. It can also be used in environments where the end-system is connected to a Local Area Network or where end systems are connected by a dedicated path or by a circuit-switched connection. 
The NS provider (more particularly, the Network Layer (NL) entity in an end-system) must provide a translation between

a. the primitives and parameters of the OSI CONS; and

b. the packets and associated fields of the X.25/PLP.

Request and response primitives are translated into packets to be transmitted across the DTE/DXE interface by the NL entity. Rece ived packets, where appropriate, are translated by the NL entity into ind ication and confirm primitives. These translations are shown in sections 6.2.2.2 through 6.2 .2 .7 .

Note: The Network Service Defin ition specifies valid sequences of primitives at a NC endpoint and val id parameter responses at the called NC endpoint to Receipt Confirmation negot iation, Expedited Data negotiation, and QOS parameter negotiation. The necessity for the NL entity to police compliance and the NL entity actions to be taken on non-compliance are a local matter and not subject to standardization.

There is also a relationship between some local mechan ism used to ident ify a particular Network Connection (NC) and a Logical Channel (LC) number used to ident ify a particular virtual circuit. This relationship is a local matter and is not discussed here.

\subsubsection{Network Connection Establ ishment Phase}

Table 6.2 shows the relationships between the primitives/parameters used during the Network Connection Establishment Phase and the packets/fields associated with the Call Setup Procedures. 
Table 6.2

CONS: $X .25 / P L P$ mapping for the network connection establishment phase

\begin{tabular}{|c|c|}
\hline CONS & X.25/PLP-1984 \\
\hline $\begin{array}{l}\text { PRIMITIVES: } \\
\text { N-CONNECT request } \\
\mathrm{N} \text {-CONNECT ind icat ion } \\
\mathrm{N} \text {-CONNECT response } \\
\mathrm{N} \text {-CONNECT confirm }\end{array}$ & $\begin{array}{l}\text { PACKETS: } \\
\text { CALL REQUEST } \\
\text { INCOMING CALL } \\
\text { CALL ACCEPTED } \\
\text { CALL CONNECTED }\end{array}$ \\
\hline PARAMETERS: & FIELDS (INCLUDING FACILITIES): \\
\hline Called Address & Called Address Extension Facil ity \\
\hline Calling Address & $\begin{array}{l}\text { Call ing Address Extension } \\
\text { Facil ity }\end{array}$ \\
\hline Responding Address & Called Address Extension Facility \\
\hline $\begin{array}{l}\text { Rece ipt Conf irmation } \\
\text { Select ion }\end{array}$ & See Note 1 \\
\hline Expedited Data Selection & See Note 1 \\
\hline QOS Parameter Set & $\begin{array}{l}\text { Throughput Class Negotiation } \\
\text { Facil ity2 } \\
\text { Min imum Throughput Class } \\
\text { Negot iation Facil ity } \\
\text { Trans it Delay Select ion And } \\
\text { Indication Facil ity } \\
\text { End-to-End Trans it Delay } \\
\text { Negot iation Facil ity }\end{array}$ \\
\hline NS-User-Data & $\begin{array}{l}\text { Call and Called User Data Field } \\
\text { Fast Select Facility } 3\end{array}$ \\
\hline
\end{tabular}

Notes to Table 6.2:

1. With in the scope of these agreements, the Receipt Confirmation Service and Expedited Data Service will not be used. Therefore, the Network Service provider will indicate the unavailability of these services even if requested by the Network Service user. To do this does not require an explicit protocol mechanism.

2. For proper operation, this optional user facil ity must also be agreed to for use on the interface.

3. For proper operation, the Fact Select Acceptance Facility must also be agreed to on the interface when accessing a packet-switched network. 


\subsubsection{Network Connection Release Phase}

Table 6.3 shows the relationships between the primitives/parameters used during the Network Connection Release Phase and the packets/fields associated with the Call Clearing Procedures.

Table 6.3

CONS: X.25/PLP mapping for the network connection release phase

\begin{tabular}{|l|l|}
\hline \multicolumn{1}{|c|}{ CONS } & \multicolumn{1}{|c|}{ X.25/PLP-1984 } \\
\hline PRIMITIVES: & PACKETS: \\
$\begin{array}{l}\text { N-DISCONNECT request } \\
\text { N-DISCONNECT ind icat ion }\end{array}$ & $\begin{array}{l}\text { CLEAR REQUEST } \\
\text { CLEAR INDICATION, RESTART INDICATION }{ }^{1}, \\
\text { CLEAR REQUEST } 2\end{array}$ \\
\hline PARAMETERS: & FIELDS (INCLUDING FACILITIES): \\
Originator and Reason & Cause Code and Diagnost ic Code Fields 3 \\
NS-User-Data & Clear User Data \\
Responding Address & Called Address Extens ion Facil ity \\
\hline
\end{tabular}

Notes to Table 6.3 :

1. Receipt of a RESTART INDICATION packet should be treated as rece ipt of a CLEAR INDICATION packet for every logical channel and then mapped to an N-DISCONNECT ind ication primitive for every act ive NC associated with the Packet Level Protocol being restarted. The Restarting Cause Code and Diagnostic Code Fields are then treated in the same manner as the Clearing Cause Code and Diagnostic Code Fields.

2. Used only when the NL entity in the end system originates an NDISCONNECT indication primit ive.

3. The combination of Cause Code and Diagnostic Fields is mapped to/from the comb ination of Originator and Reason parameters. Where the IS 8208 diagnost ic codes are not provided, all Cause/Diagnostic code comb inations can be mapped to the Orignator/Reason parameter values of "Undefined".

\subsubsection{Data Transfer Phase -- Data Transfer Service}

Table 6.4 shows the relationships between the primitives/parameters used for the Data Transfer Service and the packets/fields associated with the Data Transfer Procedures. 
Table 6.4

CONS: X.25/PLP mapping for the data transfer service

\begin{tabular}{|c|c|}
\hline CONS & X.25/PLP-1984 \\
\hline PRIMITIVES: & PACKETS: \\
\hline $\begin{array}{l}N \text {-DATA request } \\
\text { N-DATA ind icat ion }\end{array}$ & $\begin{array}{l}\text { DATA } \\
\text { DATA }\end{array}$ \\
\hline PARAMETERS: & FIELDS: \\
\hline $\begin{array}{l}\text { NS-User-Data } \\
\text { Confirmation Request }\end{array}$ & $\begin{array}{l}\text { User Data, M-bit } \\
\text { See Note } 1\end{array}$ \\
\hline
\end{tabular}

Note to Table 6.4:

1. Since the Receipt Confirmation Service is not to be provided, a Confirmation Request is not valid.

\subsection{5 Data Transfer Phase -- Receipt Confirmation Service}

This service is not provided with in the scope of these agreements.

6.2.2.6 Data Transfer Phase -- Expedited Data Transfer Service

This service is not provided with in the scope of these agreements.

\subsubsection{Data Transfer Phase -- Reset Service}

Table 6.5 shows the relationships between the primitives/parameters used for the Reset Service and the packets/fields associated with the Reset Procedures. 
Table 6.5

CONS: X.25/PLP mapping for the reset service

\begin{tabular}{|l|l|}
\hline \multicolumn{1}{|c|}{ CONS } & \multicolumn{1}{c|}{ X.25/PLP-1984 } \\
PRIMITIVES: & PACKETS: \\
N-RESET request & RESET REQUEST \\
N-RESET ind icat ion & RESET INDICATION, RESET REQUEST 1 \\
N-RESET response & none \\
N-RESET conf irm & FIELDS: \\
\hline PARMETERS: & $\begin{array}{l}\text { Cause Code and Diagnost ic Code } \\
\text { Fields } 2\end{array}$ \\
\hline
\end{tabular}

Notes to Table 6.5:

1. Used only when the NL entity in the end system originates an $\mathrm{N}$-RESET indication primit ive.

2. The comb ination of Cause Code and Diagnostic Code Fields is mapped to/from the comb ination of Originator and Reason parameters. Where the IS 8208 diagnost ic codes are not provided, all Cause/Diagnost ic code comb inations can be mapped to the Originator/Reas on parameter values of "Undef ined".

\subsubsection{Requirements for Underlying Layer}

As cited in IS 8208 , the $X .25 / P L P$ requires the following of the underly ing Layer 2:

a. low dupl ication rate;

b. low missequencing rate;

c. low undetected bit-error rate; and

d. low loss rate.

When operating in a packet-switched $(X .25)$ network environment, the underlying LAPB protocol ensures these requirements. When operating in a LAN environment, LLC 1 can be used. Considerations for LLC1 are given in DP 8881.

\subsubsection{Consideration of OSI Transport Layer Protocol Class}

It may be useful to explore the desirability of an alternative to the Transport Class 4 protocol for use over the CONS. See sect ion 7, "Transport." 


\subsubsection{Subnetwork Dependent Convergence Protocol}

In cases where an end system is required not to use the elements of the X.25/PLP-1984 needed to support the OSI CONS (e.g., when operating in a packet-switched network environment that will treat as an error the use of any of the CCITT-Specified DTE facilities), then it shall use a Subnetwork Dependent Convergence Protocol (SNDCP) to provide the necessary elements of the OSI CONS to the Network Service user. These elements involve certain aspects of the Network Connection Establ ishment Phase and the Network Connection Release Phase.

The SNDCP to be used is referred to as the Alternat ive Procedures for Network Connection Establishment and Release in DP8878. The procedures for the data transfer phase of the SNDCP, as specified in DP8878, are essentially the same as those in sections 6.2.2.4 through 6.2.2.7 of this document.

\subsubsection{Network Connection Establ ishment Phase}

The procedures for this phase are as specified in DP8878, Annex A, Section 6.1 (b) (the Alternative Network Connection Establ ishment Procedure). These procedures use:

(a) a CALL REQUEST packet containing (in addition to the packet header, the Address Fields, and other facilities):

- the Throughput Class Negotiation Facil ity if available; otherwise, the throughput of the virtual circuit must be known a priori (by way of the Default Throughput Classes Ass ignment Facility, for example), and

- a Call User Data Field contain ing three octets: a one-octet Protocol ID ident ifying the X.25/PLP-1980 SNDCP encoded X'84' (as assigned by ISO) and a two-octet Cont inuation Parameter encoded $X^{\prime} 2 D 00^{\prime}$ to indicate the first $M-b$ it sequence (MBS) of DATA packets will conta in additional Network Connect ion (NC) Establ ishment parameters;

(b) a CALL ACCEPTED packet with necessary information for proper $X .25$ subnetwork interface operat ion but without any NC Establ ishment parameters;

(c) an MBS of one or more DATA packets with the Q-bit set to 1 , sent by the or iginator of the NC Establ ishment attempt, containing all necessary NC Establishment parameters encoded according to DP8878, Annex A, Section $8.7(\mathrm{c}$ ) (th is MBS is known as an N-CR message); and

(d) an MBS of one or more DATA packets with the Q-b it set to 1 , sent by the recipient of the NC Establishment attempt (assuming NC acceptance), conta in ing a 11 necessary NC Establ ishment parameters encoded according to DP8878, Annex A, Section 8.7 (d) (th is MBS is known as an N-CC message); see section 6.2.5.2 for NC refusal. 
The transit delay of each subnetwork must be estimated.

Similar to the use of the X.25/PLP-1984m the following items are to be noted:

(a) the Network Service provider will ind icate the unavailability, during NC Establ ishment, of the Receipt Confirmation Service and the Expedited Data Service; therefore, no protocol mechanisms are necessary for the negotiation of these services; and

(b) NSAP Addresses are carried entirely in the Address Extension Fields of the $\mathrm{N}-\mathrm{CR}$ and $\mathrm{N}-\mathrm{CC}$ messages.

A Connect Response Timer may be used per DP8878, Annex A, Section $6.8(\mathrm{a})$.

Figure 6.1 shows the NC Establ ishment Phase.

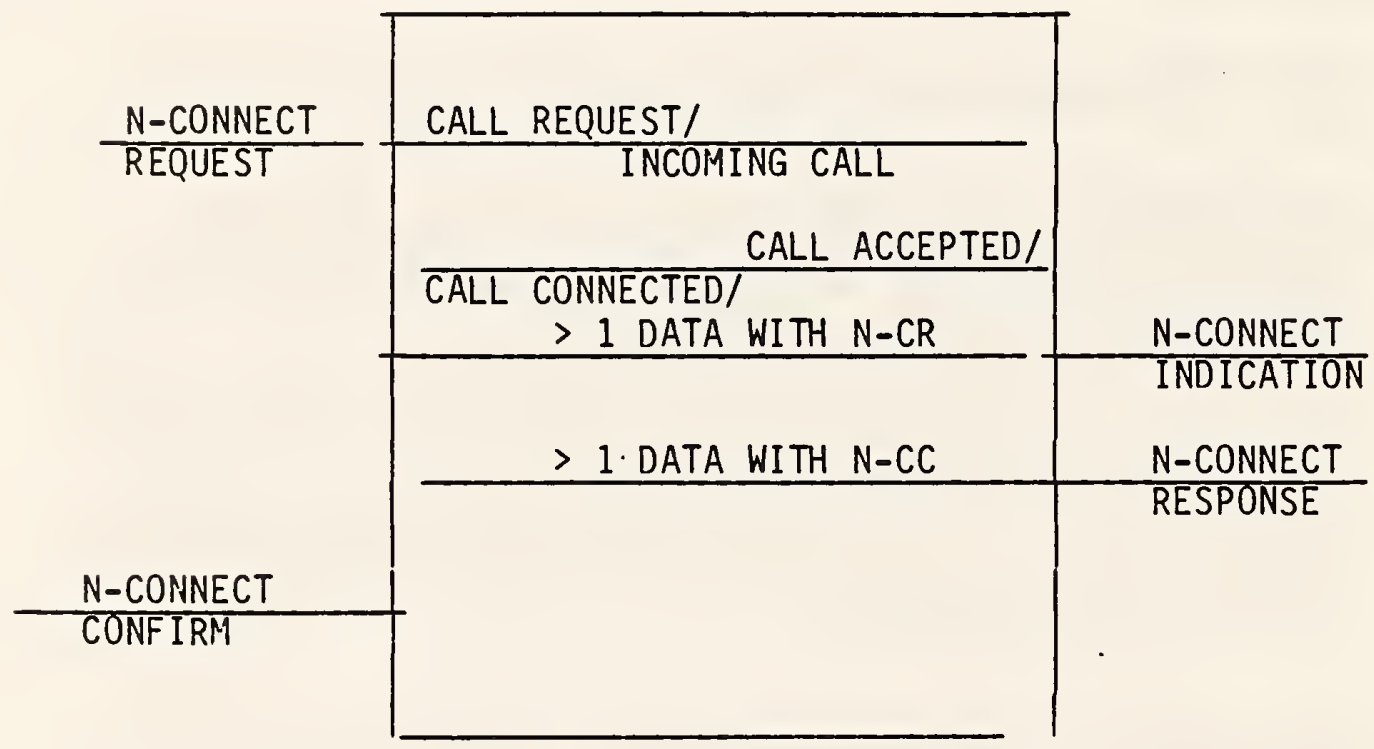

Figure 6.1 Successful NC establ ishment

\subsubsection{Network Connection Release Phase}

The procedures for this phase are as specified in DP8878, Annex A, Section 6.2 (b) (the Alternative Network Connection Release Procedure). These procedures are also used to refuse an NC establishment attempt. These procedures use:

(a) an MBS of one or more DATA packets with the Q-b it set to 1 , sent by the or iginator of the NC release, containing all 
necessary NC Release parameters encoded according to DP8878, Annex A, Section 8.8(a) (this MBS is known as an N-DR message and serves as an "invitation to clear" (that is, in itiate X.25 clearing procedures to the recipient); and

(b) the X.25 CLEAR REQUEST/INDICATION packet as indicated in DP8878, Annex A, Sect ion 8.8(b).

The above procedures apply to NC release when in itiated by the Network Service user. When in it iated by the NS provider, the N-DR message will not be sent but the Originator and Reason parameter values shall both be "undef ined."

A D isconnect Response Timer may be used per DP8878, Annex A, Section $6.8(\mathrm{~b})$.

Figure 6.2 shows the NC Release Phase.

\subsubsection{Data Transfer Phase - Data Transfer Service}

The Data Transfer Service for the X.25/PLP-1980 SNDCP is the same as for the X.25/PLP-1984 procedures (see section 6.2 .2 .4 of this document).

\subsubsection{Data Transfer Phase - Rece ipt Conf irmation Service}

As for the X.25/PLP-1984 procedures, this service is not provided with in the scope of these agreements.

\subsection{5 Data Transfer Phase - Expedited Data Transfer Service}

As for the X.25/PLP-1984 procedures, this service is not provided with in the scope of these agreements.

\subsubsection{Data Transfer Phase - Reset Service}

The Reset Service for the X.25/PLP-1980 SNDCP is the same as for the $X .25 / P L P-1984$ procedures (see section 6.2.2.7 of this document). 


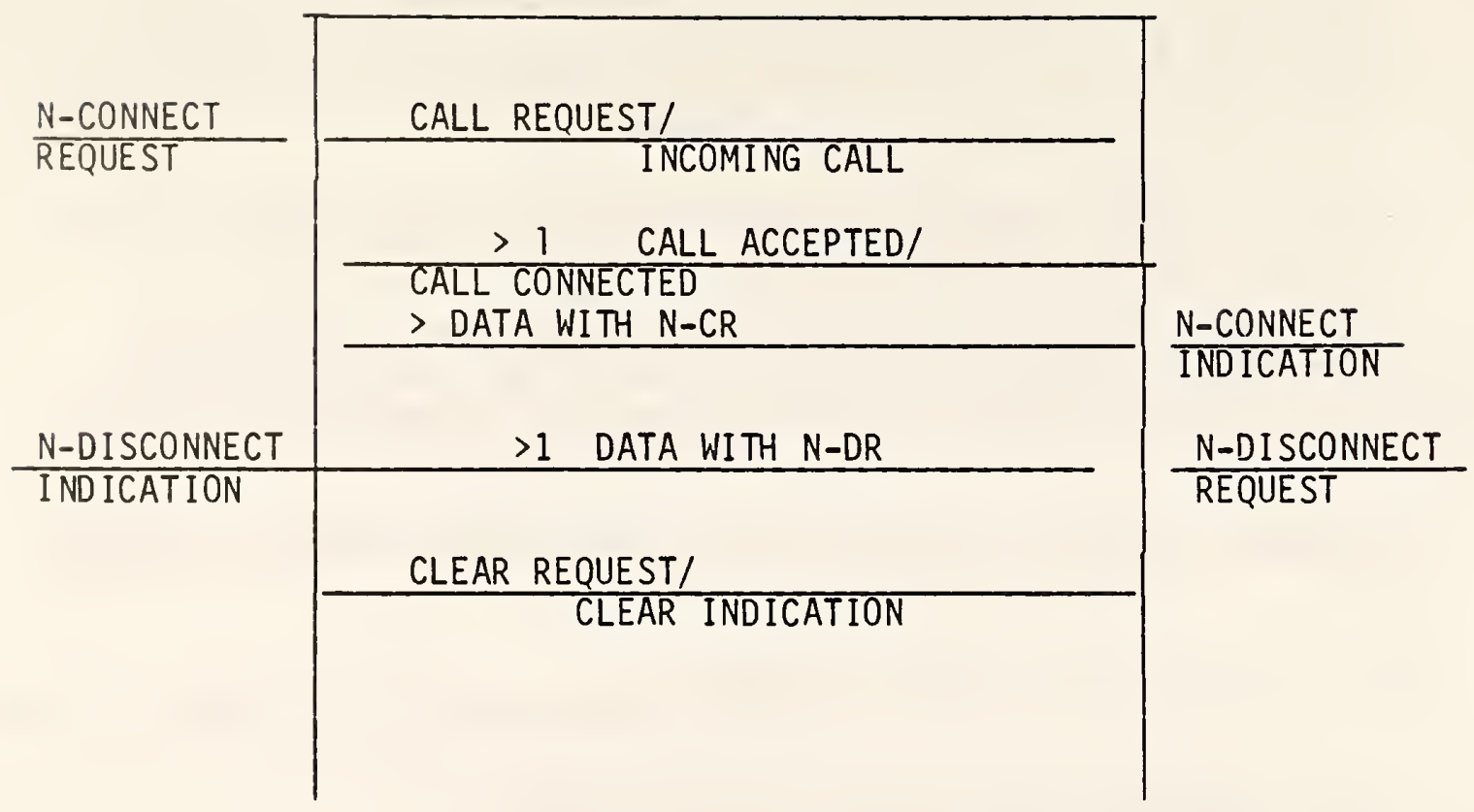

(a) NC ESTABLISHMENT REFUSAL

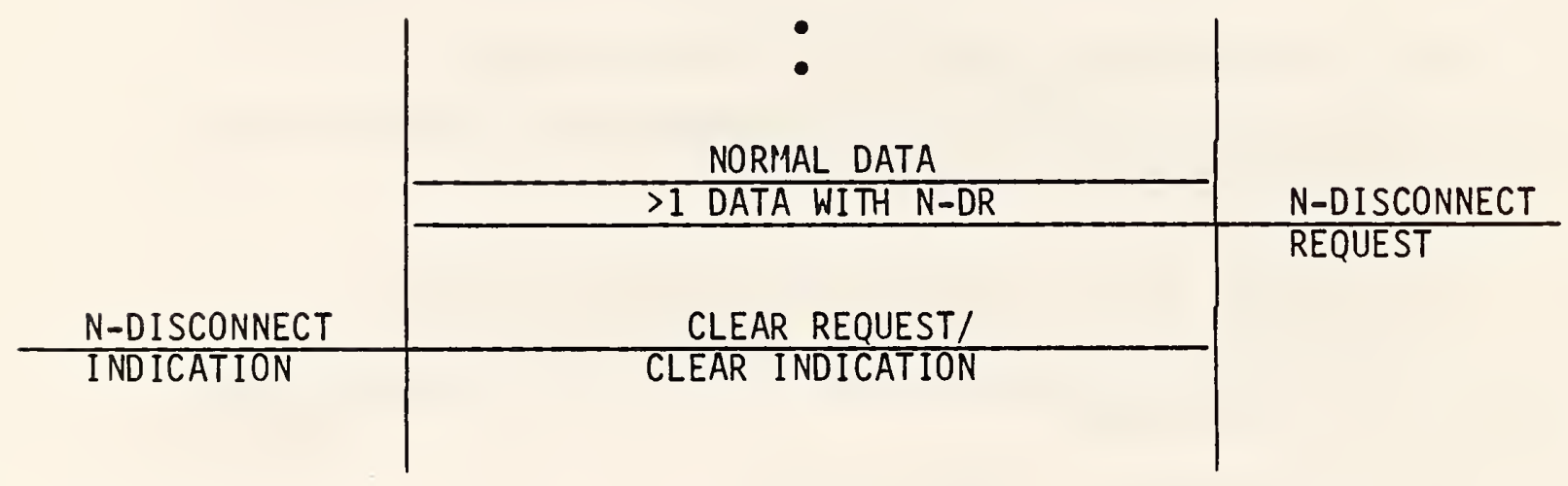

(b) NC RELEASE AFTER DATA TRANSFER

Figure 6.2 NC release

\subsubsection{Interwork ing}

Interworking between subnetworks needs to be specified. The principles of the Internal Organ ization of the Network Layer, DP 8648, need to be addressed. A generalization of these principles for the OSI CONS case is shown in Figure 6.3 . 


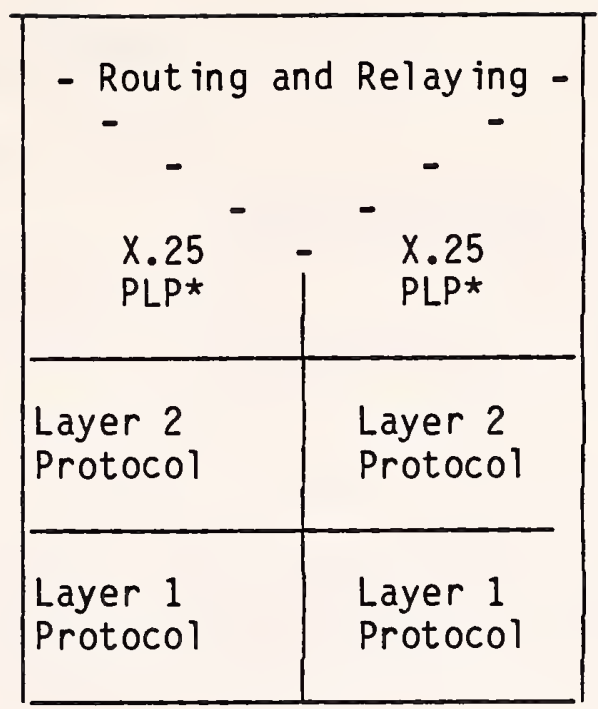

*Th is can be either X.25/PLP-1984 or X.25/PLP-1980 with an SNDCP (see Sect ion 6.2.5).

Figure 6.3 General ized interworking for OSI CONS

The detailed behavior and properties of the Interworking Un it are to be specified in future extensions of this document. DP8881 and DP 8878 discuss various aspects of interworking.

\subsection{ADDRESSING AND ROUTING CRITERIA}

The following have been agreed to.

1. Do not go beyond ISO routing standardization efforts.

2. Keep the end system as simple as possible. Put the needed compleity into the intermediate systems. Minimize memory overhead and enhance performance in the end systems.

3. Addressing and routing decisions should include the NBS OSINET that permits inclusion of private subnetworks and end systems on PDNs.

4. Permit multiple subnetwork (alternate paths) in a single intermediate system.

5. Allow expandability for additional functional ity beyond that required for a specific demonstration.

6. Support a certain minimal topology. Accommodate mult iple LANs, WANs, and private subnetworks.

7. Facilitate efficient implementation of intermediate systems. Minimize memory overhead and enhance performance of intermediate systems. 
8. The OSINET will accommodate end systems attached directly to

PDNs as one requirement.

\subsection{GENERAL ADDRESSING AND ROUTING PRINCIPLES}

The following have been agreed to.

1. Support DAD2. This supports criterion no. 1.

2. Rout ing Management

Stat ic Rout ing:

All end systems and intermediate systems will provide a local mechanism to man ipulate (and optionally create) the local rout ing table. Consistency checking, configuration, and updating of a local table with all other tables will be performed by human operators. The lack of flexibility induced by static procedures can be minimized with the use of the following aids:

- alternate static routes

- route update ut il ity

- FTAM route table distribution

- table format via X.409 (ASN-1) encoding.

Dynamic Rout ing:

Endsystem-Intermediate System - A standard that provides the functional ity of dynamic routing must be provided. The protocol referenced in the

"References" section. Item ISO 18, "Network Layer Management Protocol....", provides for this functional ity between end and intermediate systems. Th is standard is expected to be endorsed (and appropriate implementor agreements drafted) to provide this function at a $t$ ime when it reaches the "Draft Proposal" status.

\section{Intermediate system-Intermediate System}

To be determined

3. Routing Principles

The algorithm and data structures used for routing are not specified by this document. Implementors are free to perform these functions in the manner which is most appropriate for the ir system environment. However, all implementations must have the following characteristics:

a) end-systems

End-systems must recognize a destination address on a directly connected subnet and send the NPDU to the dest ination system. When an NPDU is dest ined to a system that is not on a directly connected subnet, the NPDU must be sent to an intermediate system for further routing. The end system may, but is not 
required to, choose the intermediate system used on the basis of the destination subnet.

\section{b) intermediate systems}

For static routing, intermediate systems must recognize all assigned subnetwork addresses and route NPDU's in the following way: If an NPDU is destined to an end system which is connected to the same subnet as the intermediate system, the NPDU is sent directly to the end system. If an NPDU is destined to an end system which is not connected to the same subnet as the intermediate system, the NPDU is sent to an appropriate intermediate system for further routing. If an NPDU is destined to an end system on an unknown subnet, the NPDU is discarded. If the error flag is set, an error NPDU is sent to the source address specified in the errant NPDU.

4. There is a single NSAP selector for each NSAP within an end system. For each end system NSAP, one and only one NSAP address will be used to identify the NSAP. This conforms to the requirements of DAD2 and supports criterion no. 1 to comply with current ISO standards.

5. Hierarchical NSAP addressing should be used to minimize the size of routing tables. This conforms to criterion no. 9.

6. The NSAP address used at the network service interface is based on one and only one subnetwork point of attachment (SNPA). This makes the hierarchial addressing more specific and implies that subnetwork specific addressing information is embedded in the NSAP address.

7. The encoded network protocol address information (NPAI) conveyed in the IPDU representing an NSAP address contains an encoded subnetwork address corresponding to the selected SNPA. This makes hierarchical addressing more specific as applied to addressing formats. The embedding of the subnetwork address facilitates routing and simplifies routing tables.

8. The workshop participants require control over the DSP encoding of the NSAP address.

9. NBS has obtained an IDI with value 4 from ISO for OSINET so that the AFI encoding " 47 " may be used.

10. Optionally, the NSAP addressing format should be able to support multiple network layer user entities (e.g., transport entities) within one end system. 
11. All of the above principles lead to the address format agreed upon and shown below.

\begin{tabular}{|l|l|l|l|}
\hline 47 & ISO & $\begin{array}{l}\text { NBS } \\
\text { ASSIGNED } \\
\text { ORGANIZATION } \\
\text { I.D. }\end{array}$ & $\begin{array}{l}\text { PORTION OF DSP DEFINED } \\
\text { ASSIGNED BY INDIVIDUAL ORGANIZATION } \\
\text { ADDRESSING AUTHORITIES }\end{array}$ \\
\hline
\end{tabular}

12

$2 \leq 11$

Figure 6.4 NSAP address format

12. If used in the DSP, an. NSAP Selector field is not used for routing purposes nor for locating the end system. It only locates the network layer user entity with in the end system attached through the addressed NSAP.

13. In order to fulfill the criteria in section 6.3, item 3, a standard that provides the functionality of dynamic routing must be provided. The protocol referenced under ISO, item \#18 "Network Layer Management Protocol ..." provides for th is functional ity between end and intermediate systems. This standard is expected to be endorsed to provide this function at a time when it reaches a "Draft Proposal" status.

\section{TRANSPORT}

The object of this set of agreements is to support the integration of the types of networks covered here (LANs, packet networks, other WANs) with the smallest possible set of mandatory protocol sets, in accordance with the other agreements already reached. Nothing here shall preclude vendors from implementing protocol suites in add it ion to the ones described in this document. Two connection or iented transport classes have been ident if ied for implementation (class 0 and class 4 ). In addition, there is interest among a limited set of participants in implementation of a connectionless transport protocol. Transport class 4 (over CLNP) has been endorsed for general communication between private systems. Transport class 0 (over $X .25$ ) is used for commun icat ion with publ ic (i.e. PT\&T and RPOA) MHS systems operating in accordance with the CCITT X.400 series recommendations. Communicating ent it ies between private MHS systems over an X.25 network can, by negot iation or bilateral agreement, agree to use transport class 0 . The connectionless transport protocol can be used with transaction-type implementations.

\subsection{TRANSPORT CLASS 4}

\section{1 .1 Transport Class}

The following agreement has been reached with respect to this protocol.

Class 4 will be used with the required implementation of the 31 bit sequence space and 16 bit window size. The full protocol will be used including expedited data and negotiation at connection establ ishment. These two functions were not implemented for the 1984 NCC demonstrations. 


\subsubsection{Protocol Interpretation}

According to the ISO transport specification, a disconnect request is issued in response to a connect request when the maximum number of transport connections is reached or exceeded.

\subsubsection{Rules for Negotiation}

1. In general, the ISO rules for negotiation will be used, specifics follow.

2. All implementations will send the $16 / 31$ window size/sequence space in the CR TPDU. Implementations must all provide the $16 / 31$ ISO option. Implementations must be able to accept the 4/7 in CR TPDU.

3. The ISO TPDU size is 128 to $8 \mathrm{~K}$ octets, always negotiated downward. The ISO rules are to be followed, allowing any valid size in the CR TPDU. TPDU size negotiation is a local implementation issue. Each vendor will decide how it is implemented in their end system.

4. The security parameter is optional and user defined in the ISO specification. Implementations should not send the security parameter in the CR TPDU; if received it should be ignored.

5. Both transports must agree to not use checksum, according to the ISO specifications. Requesting its use is an implementation choice. All implementations must be able to operate with checksum if requested.

6. Use of acknowledgement time parameter is optional in Iso 8073 . If an implementation is operating any policy which delays the transmission of AK TPDUs, the maximum amount of time by which may single AK TPDU may be delayed shall be indicated to the peer transport service provider using the acknowledgement time parameter. The value transmitted should be expressed in units of milliseconds and rounded up to the nearest whole millisecond.

7. Throughput, priority, and transit delay are optional in the ISO specification. Do not send in the CR TPDU; ignore in the CC TPDU.

8. User data in the CR TPDU and the CC TPDU are optional. No implementation should send; all implementations must be prepared to receive. 


\subsubsection{Retransmission Timer}

It is recommended that the value used for the retransmission timer

be based upon the round-trip delay experienced on a transport

connection. The implementation should maintain, and continually update, an estimate of the round-trip delay for the TC. From this estimate, a value for the retransmission timer is calculated each time it is started. An example technique for maintaining the estimate and calculating the retransmission timer is described below. Further information on similar techniques may be found in the literature [Edge 84, Jain 85, Mill 83].

The value of the retransmission timer may be calculated according to the following formula:

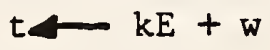

In this formula, $E$ is the current estimate of the round-trip delay on the transport connection, $w$ is the value of the acknowledgement time parameter received from the remote transport service provider during connection establishment, and $k$ is some locally administered factor.

A value for $k$ should be chosen to keep the retransmission timer sufficiently small such that lost TPDUs will be detected quickly, but not so small that false alarms are generated causing unnecessary retransmission.

The value of $\mathrm{E}$ may be calculated using an exponentially weighted average based upon regular sampling of the interval between transmitting a TPDU and receiving the corresponding acknowledgement. Samples are taken by recording the time of day when a TPDU requiring acknowledgement is transmitted and calculating the difference between this and the time of day when the corresponding acknowledgement is received. New samples are incorporated with the existing average according to the following formula.

$$
E-E+(1-a) S
$$

In this formula, $S$ is the new sample and alpha is a parameter which can be set to some value between 0 and 1 . The value chosen for alpha determines the relative weighting placed upon the current estimate and the new sample. A large value of alpha weights the old estimate more heavily causing it to respond only slowly to variations in the round-trip delay. 
A small value weights the new sample more heavily causing a quick response to variations. (Note that setting alpha to 1 will effectively disable the algorithm and result in a constant value for $E$ being that of the in itial seed.)

If alpha is set to $1-2^{-n}$ for some value of $n$, the update can be reduced to a subtract and shift as shown below.

$$
E \leftarrow E+2-n(S-E)
$$

When sampling, if an AK TPDU is received which acknowledges mult iple DT TPDUs, only a single sample should be taken being the round-tr ip delay experienced by the most recently transmitted DT TPDU. Th is attempts to minimise in the sample any delay caused by the remote transport service provider withholding AK TPDUs.

\subsubsection{Keep-Al ive Funct ion}

The Class 4 protocol detects a failed transport connection by use of an 'inactivity timer'. This timer is reset each $t$ ime a TPDU is received on a connection. If the timer ever expires, the connection is terminated.

The Class 4 protocol maintains an idle connection by periodically transmitting an AK TPDU upon expiration of the 'window $t$ imer'. Thus, in a simple implementation, the interval of one transport entity's window timer must be less than that of its peer's inactivity timer, and vice versa. The following agreements permit commun icating transport ent it ies to ma inta in an idle connection without shared information about timer values.

1. In accordance with ISO 8073 , clause 12.2.3.9.a, all implementations must respond to the receipt of a duplicate AK TPDU by transmitting an AK TPDU containing the 'flow control confirmation' parameter.

2. Implementations must always transmit duplicate AK TPDUs on expiration of the local window timer (see ISO 8073, clause 12.2.3.8.1). Rece ipt of th is TPDU by the remote transport entity will cause it to respond with an AK TPDU containing the 'flow control confirmation' parameter. When this is received by the local transport entity, it will reset its inactivity timer. See figure 7.1 .

3. It is a local matter for an implementation to set the intervals of its timers to appropriate relative values. Specifically:

a) The window timer must be greater than the round-trip delay. See section 7.1.4.

b) The inactivity timer must be greater than two times the window timer; and should normally be an even greater multiple if the transport connection is to be resilient to the loss of an AK TPDU. 
A duplicate AK TPDU (see Figure 7.1) is one which contains the same values for YR-TU-NR, credit, and subsequence number as the previous AK TPDU transmitted. A duplicate AK TPDU does not acknowledge any new data, nor does it change the credit window.

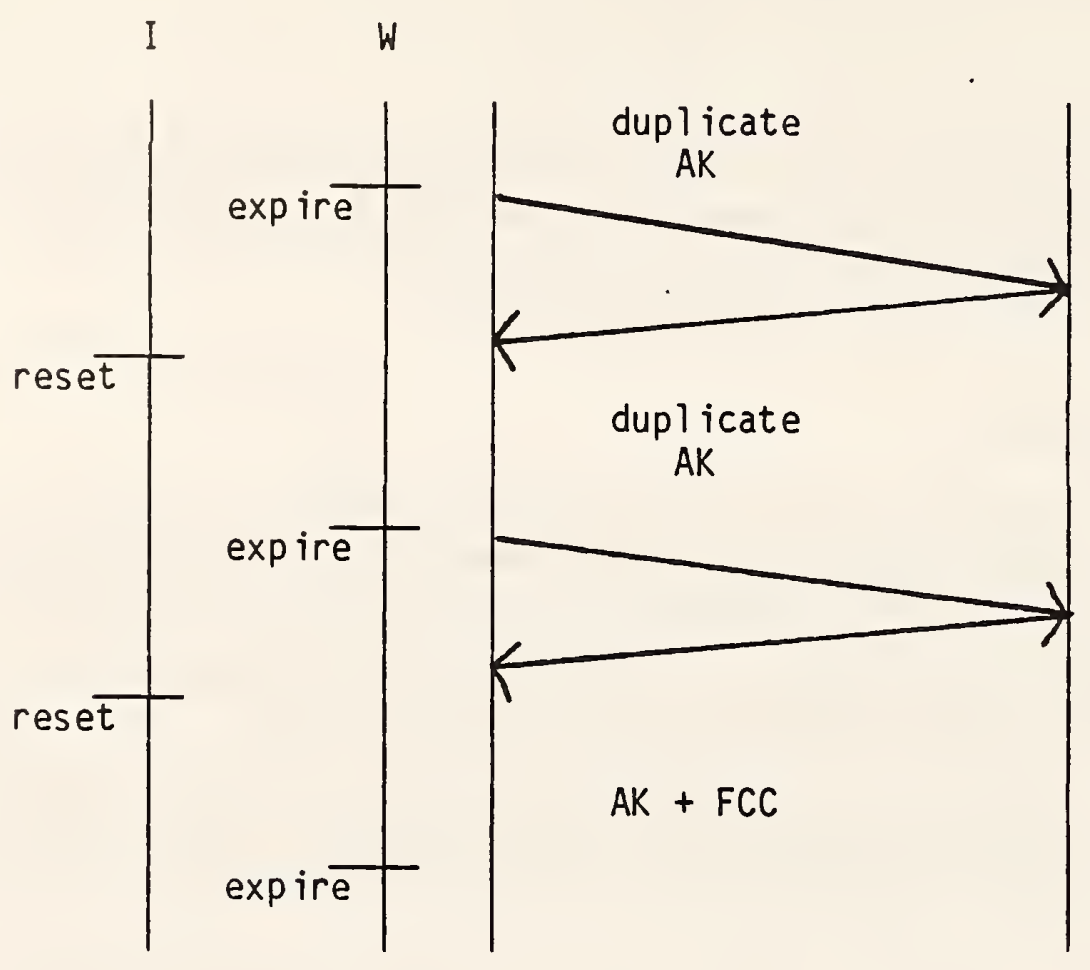

Figure 7.1 AK exchange on idle connection

\subsection{TRANSPORT CLASS 0}

\subsubsection{Transport Class}

Transport class 0 over $X .25$ is mandatory (see $X .400$ ) for use in commun icating with public IHS systems operating in accordance with the CCITT X.400 series recommendations. The purpose of the agreements concerning transport class 0 is to allow connection to these public services. Transport class 0 over $X .25$ can also be used in commun icat ing between PRMDs, but it is agreed that Transport Class 4 and CLNP over all types of lower layer networks allows a larger range of interoperation and is recommended. 


\title{
7.2.2 Protocol Interpretation
}

Transport class 0 is a relatively simple protocol providing little opportunity for conflicting interpretations. A few relevant agreements follow.

1. The Disconnect Request (DR) TPDU shall be 1 imited to the first seven octets - "LI" plus "fixed part".

2. The Error (ER) TPDU may be used at any time and upon receipt requires that the recipient disconnect the network connection (and by extens ion the transport connection).

\subsubsection{Rules for Negot iation}

1. The ISO rules for negotiations will be used.

\subsection{CONNECTIONLESS TRANPORT}

Document ISO IS 8072/DAD1 is the Transport Service Def in it ion covering Connect ionless-mode Transmiss ion. Document ISO DIS 8602 is the Protocol for providing the Connection less-mode Transport service.

\section{SESSION}

Session services are defined to meet the needs of many applications. The Session service is defined in ISO 8326 (CCITT X.215) and the session protocol is defined in ISO 8327 (CCITT X.225). The general agreements about the use of session are documented below in section 8.1 , followed by agreements that are related to specific applications.

\subsection{GENERAL}

The services of the Session kernel functional unit are used as specified in the standard.

\author{
Session Connection \\ Data Transfer \\ Orderly Release \\ Provider Abort \\ User Abort
}

Bas ic concatenation is required by the session protocol standard. Extended concatenation is not required and can be refused using the normal negotat ion mechanisms provided by the session protocol.

Session segmenting is not required and can be refused using the normal negotiation mechanisms of the session protocol.

Reuse of a transport connection is not required and can be refused us ing the normal negotiation mechan isms of the session protocol.

The use of transport expedited is as stated in the session protocol specification. That is, if transport expedited is available it must be used.

The maximum length of the reflect parameter values parameter in the S-P Except ion-Report is 1024 octets. 
The user data parameter on S-CONNECT SPDU will allow unlimited length user data as specified in the proposed Session draft addendum. Th is is due to the fact that the comb ined P-CONNECT, A-ASSOCIATE, and F-INITIALIZE may exceed the existing 512 byte 1 imit.

\subsection{SESSION REQUIREMENTS FOR FTAM}

The phase 2 FTAM requires the following functional un its in add ition to those specified in section 8.1 .

\section{Functional Units}

Duplex
Session Services

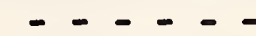

Note: Implementation of the Resynchronize functional un it is highly recommended since the F-CANCEL service may be ineffect ive when mapped to S-DATA.

\subsection{SESSION REQUIREMENTS FOR MESSAGE HANDLING}

The MHS application requires the following functional un its in addition to those specified in section 8.1 .

\begin{tabular}{ll} 
Functional Units & Session Services \\
\hline Exceptions & User Except ion Report ing \\
& Provider Except ion Reporting \\
Act ivity Management & Act ivity Start \\
& Act ivity Resume \\
& Act iv ity End \\
& Act iv ity Interrupt \\
& Act ivity Discard \\
& Please Tokens \\
& Give Tokens \\
& Give Control \\
& Give Tokens \\
Half-duplex & Please Tokens \\
& Minor Synchron izat ion Point \\
Minor Synchron ize & Give Tokens \\
& Please Tokens
\end{tabular}

Note: Restricted use is made by the RTS of the session services implied by functional units selected. Specifically,

- NO use is made of S-TOKEN-GIVE

- S-PLEASE-TOKENS on ly asks for the data token. 
The following additional points should be noted.

- In S-CONNECT, the Synchron izat ionPointSerial Number should not be present.

- Format of the SessionConnectionID is described in Version 3 of the X.400-Series Implementor's Guide.

\section{SERVICE ACCESS POINTS AND SELECTORS}

\subsection{UPPER LAYER AGREEMENTS}

The following upper layer address ing agreements have been reached.

1. The comb ination of NSAP address, TSAP selector, SSAP selector, PSAP selector and PSAP address must be unique to ident ify an application ent ity.

2. It is implicitly agreed that the procedure followed for the ass ignment of NSAP addresses insures that they are globally un ique.

3. The assignment of TSAP, SSAP, and PSAP selectors is a local end system issue and the values are administered locally.

4. SSAP selectors are encoded as a string of octets, the mean ing of which is known only to the local system. The length of the string cannot exceed 16 octets.

5. PSAP selectors are encoded as a string of octets, the meaning of which is known only to the local system. The length of the string cannot exceed 16 octets.

\subsection{TRANSPORT CLASS 4 SERVICE ACCESS POINTS OR SELECTORS}

1. The existing encoding for TSAP selectors in the CR and CC TPDU'S will be maintained. The TSAP selector field in these TPDU's shall be encoded as a variable length field, two octets in length, and will be interpreted as a bit string (not an integer).

\subsection{TRANSPORT CLASS O SERVICE ACCESS POINTS}

For communicating with publ ic MHS systems, Section 5 of $X .410$ specifies the use and format of TSAP ident if iers. 


\section{ISO FILE TRANSFER \& ACCESS MANAGEMENT PROTOCOL}

The following general agreements have been reached with respect to this protocol.

1. FTAM will be implemented in at least two phases. Phase 1 agreements are contained in section 10.1 and are devised for the MAP2.1 specification. Phase 2 agreements are contained in section 10.2 and are devised at the request of the Corporation for Open Systems for 1987 product implementations.

2. The phase 1 FTAM implementation specification is based on the second ISO draft proposal. The phase 2 FTAM specification is based on the DIS and later will be based on the IS.

\subsection{PHASE 1 FTAM IMPLEMENTATION SPECIFICATION}

\subsubsection{Phase 1 FTAM Services}

The following subset of file transfer services has been agreed to.

$$
\begin{aligned}
& F \text { - INITIALIZE } \\
& F \text { - SELECT } \\
& F \text { - OPEN } \\
& F \text { - READ, F-WRITE } \\
& F \text { - DATA-END } \\
& F \text { - TRANSFER-END } \\
& F \text { - CLOSE } \\
& F \text { - DESELECT } \\
& F \text { - U-ABORT, F-P-ABORT } \\
& F \text { - TERMINATE }
\end{aligned}
$$

(The implementation of F-CANCEL is optional.)

Both F-READ and F-WRITE will be implemented. There is at most one F-OPEN and at most one F-READ or F-WRITE per file selection; more than one file may be selected sequentially over the lifet ime of a connection. A Session connection is established at the beginning of a file activity and terminated when the file transfer connection is released. 
The following 1 imited management subset of services has been agreed to.

$$
\begin{aligned}
& F \text { - CREATE } \\
& F \text { - DELETE } \\
& F \text { - READ-ATTRIBUTE }
\end{aligned}
$$

(Note that this subset is complete with respect to the ISO 1 imited file management functional un it.)

The user correctable file service and the storage subset of the virtual

filestore attribute group will be implemented.

\subsubsection{Phase 1 File Attributes}

Phase 1 FTAM implementations will negotiate for the use of Storage Attributes.

The following file attribute agreements have been reached.

\section{FILENAME}

DP8571 Part 2 Section 11 minimum range for file name will be supported (1-8 characters). No maximum length or format restrictions. Any system which does not support extended file name characteristics would reject the (select, create, delete) request of such a file name.

File names will cons ist of upper case characters and numbers. The first character of a filename may not be a number.

\section{ACCESS STRUCTURE TYPE}

The access structure type will be unstructured. Flat and hierarchical are not used.

3. PRESENTATION CONTEXT NAME

Two structures have been approved.

VARCRLF: Text format. Each 1 ine is variable length and must be terminated by a CRLF pair. Line length is 1 imited to 250 characters plus CRLF. Form feed is excluded. CR and LF cannot occur separately. The ISO 646 character set will be used.

UNDEF : Bit string encoding. No embedded structure is recognized.

4. CURRENT FILESIZE

Value represented as conformant with second ISO draft proposal.

5. REQUESTED ACCESS

This attribute will support read, replace, read attribute, and delete file. 
6. CURRENT ACCESS STRUCTURE TYPE

The current access structure type will be unstructured.

7. CURRENT PRESENTATION CONTEXT

This attribute is the same as PRESENTATION CONTEXT, i.e., ASCII or binary.

\section{FUTURE FILESIZE}

This is a provider option. Value represented as conformant with second ISO draft proposal.

9. DATE AND TIME OF CREATION

This is a provider option. The value is represented as conformant with second ISO draft proposal. Resolution and accuracy are implementation dependent.

\subsubsection{ISO Deviations and Selections}

1. (DP8571/3 8.1 Table 1) Implementation of the F-CANCEL service for non-recoverable errors in the read and write functional un its is optional. An implementation which receives an F-CANCEL indication may issue an F-ABORT.

2. (DP8571/3 8.2 1b) There will be no implementation of the grouping functional unit. The concatenation constraint on establishing and releasing a file open regime is not supported.

3. (DP8571/3 14.1.2.5) F-INITIALIZE The value of the Service Type parameter chosen for implementation is "user correctable service".

4. (DP8571/3 14.1.2.6) F-INITIALIZE The value of the Service Class parameter chosen for implementation is "file transfer class".

5. (DP8571/3 14.1.2.7) F-INITIALIZE The value of the Functional Un its parameter chosen for implementation is the set "read", "write" and "limited file management".

6. (DP8571/3 14.1.2.8) F-INITIALIZE The value of the Attribute Groups parameter chosen for implementation is "storage".

7. (DP8571/3 14.1.2.9) F-INITIALIZE The value of the Rollback Availability parameter chosen for implementation is "no rollback".

8. (DP8571/3 14.1.2.11) F-INITIALIZE The values of the Presentation Context Name parameter chosen for implementation are defined in section 10.1 .2 of this document. 
9. (DP8571/3 17.1.2.2) F-OPEN The values of the Processing Mode parameter chosen for implementation are "read" or "replace".

10. (DP8571/3 17.1.2.3) F-OPEN The Presentation Context parameter must be present to indicate the context chosen for the transfer. (Th is is due to the lack of a presentation layer implementation at th is $t$ ime.)

11. (DP8571/3 24.3.) F-DATA will be encoded as:

IDENTIFIER: context spec if ic tag value [55]

Length: (Length of OCTETS)

Contents: (octets)

There is an expl ic it F-DATA PDU because of the direct FTAM/Sess ion mapp ing.

The ASN.1 def in it ion of F-DATA is:

F-DATA request $::=\operatorname{data[55]IMPLICIT~OCTETSTRING~}$

12. The ASN.1 type "ISO646String" will be used instead of ASN.1 type

"GraphicString" in the abstract syntax. Wherever "GraphicString" is specificed in the abstract syntax, the abstract syntax is modified to specify "IS0646String."

13. The PresentationContext attribute on the SELECT/CREATE request is restricted to a sequence of only one PresentationContextName.

14. PresentationContextName parameters will be encoded as:

PresentationContextName $\quad::=$ [Application 13] PrintableString.

15. The abstract syntax for F-INITIALIZE will be:

F-INITIALIZE request : := SEQUENCE

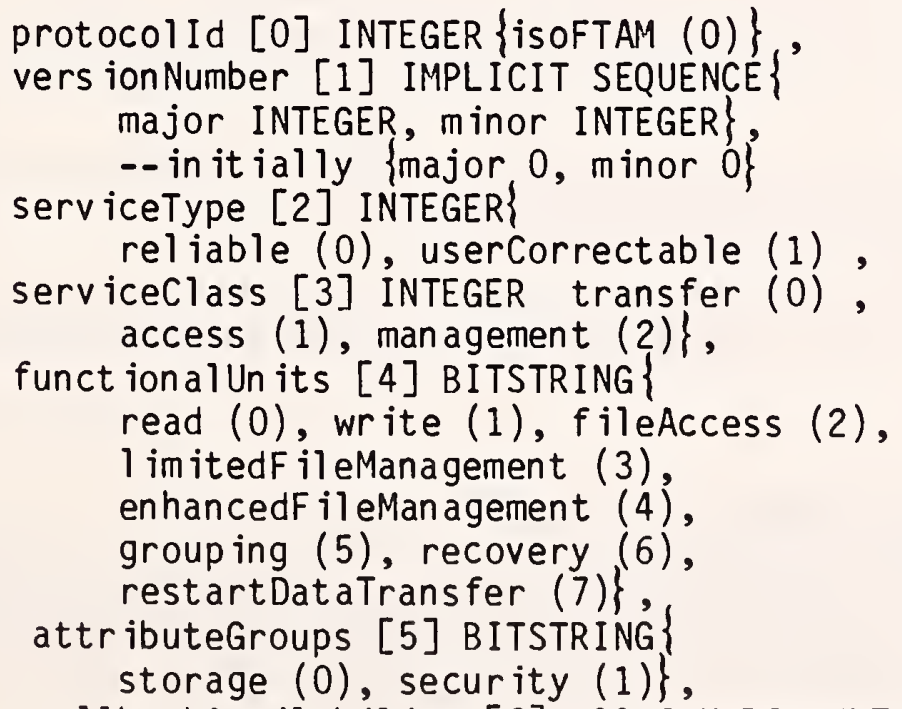

SEQUENCE OF Presentat ionContextName OPTIONAL 


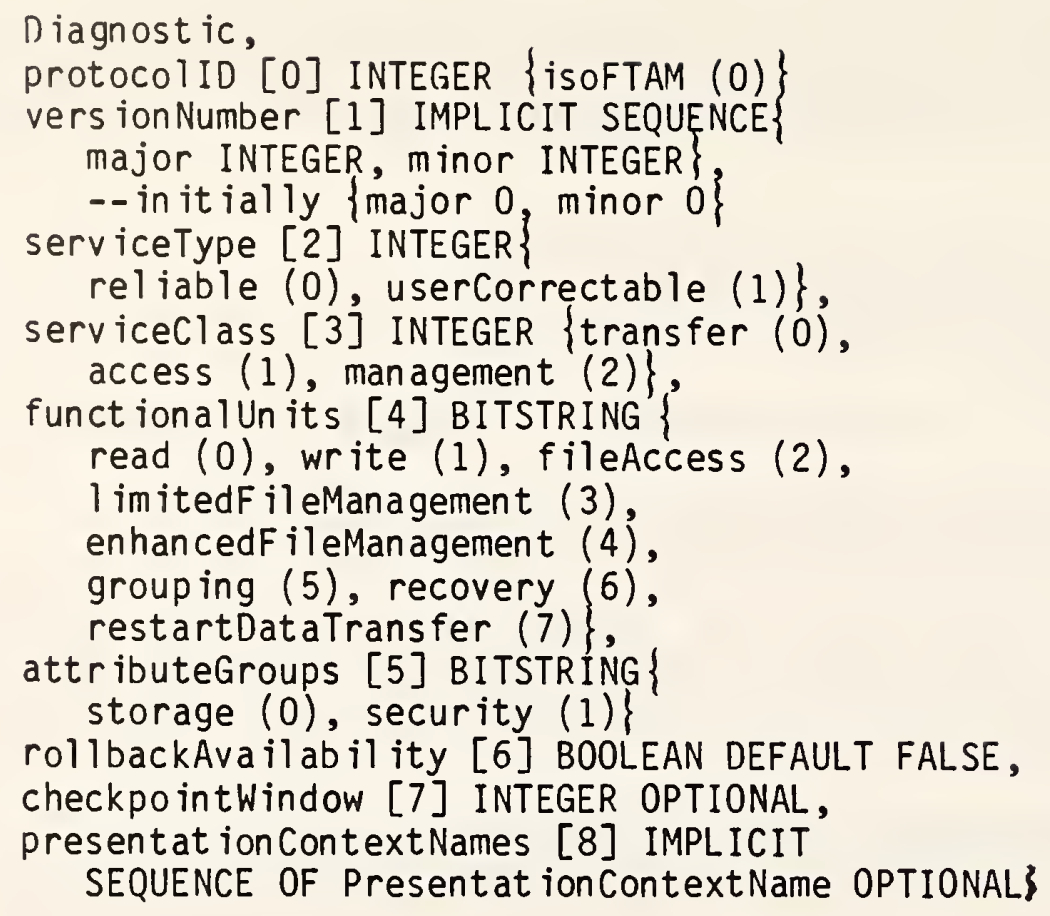

16. The PresentationContextName parameter on the F-INITIALIZE request and response will be a sequence of presentat ion context names. The corrected abstract syntax for this parameter will be:

PresentationContextNames [10] IMPLICIT SEQUENCE OF PresentationContextName OPTIONAL,

The value of this parameter, if present, will establish 1 imits for the life of the FTAM association.

17. The PresentationContextName parameter on the F-OPEN request and F-OPEN response will be mandatory instead of optional.

18. Phase 1 FTAM products will use the state mach ine in the FTAM second ISO draft proposal part 4 , except where explicitly specified differently in these agreements.

\subsubsection{Further Implementation Details}

The following recommendations are not specified by the standard but are required to be agreed upon in order to insure that different implementations work together smoothly. 
1. The FTAM implementation is mapped directly to Session services and does not incorporate the use of CASE at this time. The F-INITIALIZE request is embedded in the S-CONNECT request and the F-INITIALIZE response is embedded in the S-CONNECT response, not sent as an S-DATA request after the Session has been established.

If the F-INITIALIZE response is a pos it ive confirmation, the F-INITIALIZE response is mapped onto S-ACCEPT. Otherwise, is is mapped onto S-REFUSE.

2. $F-U-A B O R T$ and $F-P-A B O R T$ are mapped to S-DATA requests. The rece iver of an F-U-ABORT or an F-P-ABORT must issue an S-U-ABORT request. This is due to a possible S-RELEASE collison should both entries wish to abort simultaneously.

3. Implementations should be able to parse all valid second ISO draft proposal optional parameters if they are present in the PDU. Only those optional parameters specif ied in the agreements are required to be supported for request and response PDUs.

4. All second ISO draft proposal optional parameters ident if ied in these agreements as mandatory must be supported. If these parameters are not present, their semantics are a local issue and, further, the request should not be refused.

5. Error Handling and Diagnost ic

Action that is permitted by the standard is not restricted in this implementation. Implementations may return a response $w$ ith a negative diagnostic or issue an F-U-ABORT request.

6. Rollback

"No rollback" impl ies that in case of failure, the status of the involved files is unpredictable, i.e., the parties may choose, in the ir implementations, if they want to leave as is the partially transferred file or cancel it.

However, in order to avoid dangling conditions, after a failure (i.e., after an F-U-ABORT or F-P-ABORT) it is recommended, whatever the choice, to always put the files in a status which does not prevent further access both from remote and local systems.

7. Concurrency

No concurrency rules are adopted since the file attributes govern ing them will not be supported. Therefore, each implementat ion may choose the degree of concurrency on the local files, as a local matter. However, in order to min imize possible source of errors, the following implementation rules are recommended.

a. A file may be involved in several transfers simultaneously only if accessed in reading mode.

b. If a file is involved in a transfer in writing mode, any other request of access to that file (either for Read or Write) should be rejected. 
c. A file cannot be modified by local users while it is involved in a transfer operation (either in Read or Write). (As a practical matter, this may be very difficult to implement, regardless of the protection mechan isms provided by the local operating system.)

8. The ISO ASN.1 sytax will be used for encoding of PDU headers.

9. The indef in ite length style of ASN.1 encoding is not supported.

10. The maximum PDU length is 1024 octets.

11. If a read attribute request specifies an NBS optional attribute that is not supported by an implementation's virtual filestore, then that attribute will not be returned in the Read Attribute response. It is recommended that an implementation return an appropriate diagnost ic in this situation (e.g., 400, "attribute non-existent").

12. In the FTAM second ISO draft proposal, the parameter DIAGNOSTIC is def ined as an implicit sequence of diagnostics. The order of the diagnostics is arbitrary and has no sign if icance.

13. If a Responder receives an S-CONNECT indication, and the user data carried on that indication are "corrupted" (i.e., decoding the PCI results in an unrecoverable error), then

1) If the user data can be ident ified as an F-INITIALIZE request, the Responder should respond with an F-INITIALIZE response (-) or F-P-ABORT with diagnost ic conveying an unrecoverable error. This is to be carried on an S-CONNECT response (-) (i.e., rejecting the connection).

The Responder should further clarify the error in "further details field of diagnost ic".

Parameters on the F-INITIALIZE response should be reflected where appropriate; where th is is not possible, default values should be chosen by the Responder where mandatory parameters are required and cannot be reflected.

2) If the user data cannot be ident if ied as an F-INITIALIZE request, the Responder should respond with an S-II-ABORT with reason code appropriate to the error.

\subsection{PHASE 2 FTAM IMPLEMENTATION SPECIFICATION}

\subsubsection{Assumptions}

1. Implementations will be based on the ISO 8571 DIS version of FTAM. When the IS text is approved following the close of DIS ballots the agreements will be modified as necessary to meet the IS specifications. 
2. FTAM Protocol machines must be able to parse and process up to $7 \mathrm{~K}$ octets of File PCI and FTAM user data (including grouped FPDUs) as they would be encoded with the ASN.1 Bas ic Encoding Rules. It is recommended, however, that Presentation user data not be restricted in size.

3. In order to maximize interoperabil ity it is important for implementations of FTAM service providers not to restrict unnecessarily the service user's ability to generate arbitrary file service requests, as otherwise they may not be able to work with FTAM Responders whose operation is constrained by their mapping of the FTAM virtual filestore to the ir local filestore. For example, error procedures should only be invoked at the actual occurence of an error, not at the specification of options which might result in an error.

4. Implementations should be able to parse all valid DIS optional parameters if they are present in the PDU. Only those optional parameters specified as mandatory in these agreements are required to be supported for request and response PDUs. If these parameters are not present, it is a local implementation issue to assign a default value. A request should not be refused if a parameter which is optional in the FTAM standard, but is mandatory in these agreements, is not present.

5. Cons ideration of any standarized service interface is outs ide the current work items of the FTAM SIG.

\subsubsection{Presentation Agreements}

The following Abstract Syntaxes are supported:

- ISO 8571-FTAM

- ISO 8571-FADU

- ISO 8650-ACSE1

- NBS-AS1

- NBS-AS2

- NBS-AS3

A registration mechan ism will be determined to provide registered names for these syntaxes.

If the presentation context management functional un it is available, it is possible to use P-ALTER-CONTEXT to negotiate the use of an abstract syntax. 


\subsubsection{FTAM Service Type Agreements}

The user correctable service level (excluding Recovery and RestartDataTransfer functional units) will be implemented. There is no requirement to implement

the error recovery protocol mach ine.

\subsubsection{Service Class Agreements}

Implementation of the following service classes is defined.

- File Transfer

- File Access

- File Management

- File Transfer and Management

- Unconstrained

\subsubsection{Functional Un it Agreements}

Implementation of the following functional units is defined.

- Kernel

- Read

- Write

- File Access

- Limited File Management

- Enhanced File Management

- Grouping

\subsubsection{File Attribute Agreements}

1. A value for file name and contents type will always be available. Only the kernel group of attributes is required.

2. Requests for an attribute value shall always return one of the following:

(a) An actual file attribute value.

(b) A value indicating that the attribute value is not available at this time. Optionally a diagnostic may be provided indicating that the attribute is not supported.

The set of file management-related diagnostics will be maintained.

3. If the optional Storage Group is implemented, the following attribute must have an actual value available:

- Permitted Actions.

4. If the optional Security Group is implemented, the following attribute must have an actual value available:

- Access Control. 
5. Implementation of the private group is not specified.

6. Contents Type attribute is 1 imited to the Document Type Name form.

7. A minimum range for filename values is required (1-8 characters). No maximum length or format restrictions apply. A system which does not support multi-component filenames or extended filename characteristics may reject a request involving such a filename. All systems must be able to interpret single component filenames. Requests using single component filenames will be responded to using single component filenames. Responses to requests involving two or more component filenames are not defined here but may be interpreted via bilateral or other external agreement. Use of filenames with multiple components is discouraged.

\subsubsection{Document Type Agreements}

The following document types are defined.

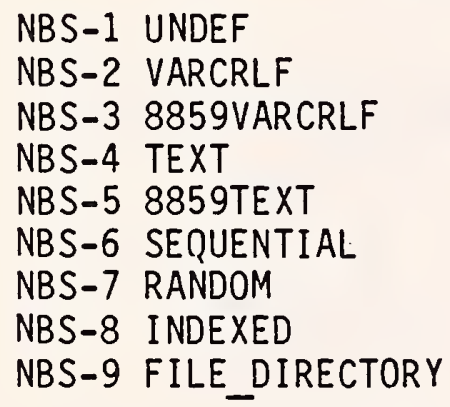

Part of the ongoing work of the FTAM SIG is to define, discuss and incorporate other file types. Detailed document type definitions are given in APPENDIX D.

Document type Names:

- DTN ::= DTName | DTName params

DTName $::=$ OBJECT IDENTIFIER

params ::=:param | :param params

param ::= Primtype | PrimType, param

$$
\begin{aligned}
& \text { PrimType : }=\text { INT }-\langle n \star\rangle \\
& \qquad \mid \begin{array}{l}
\text { BIT }-\langle n 2\rangle \\
\text { IA5 }-\langle n 1\rangle \\
8859-\langle n 1\rangle \\
\text { OCT }-\langle n 1\rangle \\
\text { UTC } \\
\text { GEN } \\
\text { NULL } \\
\text { BOOL } \\
\text { FLOAT }-\langle n 3, n 4\rangle
\end{array}
\end{aligned}
$$


$\langle n 1\rangle$ - Maximum number of characters/octets in string.

$\langle n 2\rangle$ - Number of bits in string (i.e., nonvarying).

$\langle n 3\rangle$ - The minimum number of bits required to be mantissa

for relative precision.

$\langle n 4\rangle$ - Number of bits required to represent unbiased integer exponent.

$\left\langle n^{\star}\right\rangle$ - Number of octets required to represent in $2^{\prime}$ 's complement format the largest integer to be passed.

The primitive data types and min imal size range which an implementation must accept are given in the following table.

Table 10.1 FTAM primit ive data types

\section{PRIMITIVE DATA TYPE}

ASN.1 INTEGER

ASN.1 Bit String

ASN.1 IA5String

NBS-AS1 8859String

ASN.1 OCTETSTRING

ASN.1 BOOLEAN

ASN.1 NUULL

ASN.1 General ized Time

ASN.1 Universal Time

NBS-AS1 Float ing Point

\section{REPRESENTATION}

IN PARAMETER

INT $\left\langle N^{\star}\right\rangle$

BIT $\langle$ N2>

IA5 $\langle$ N1>

$8859\langle\mathrm{~N} 1\rangle$

OCT $\langle$ N1>

BOOL

NULL

GEN

UTC

FLOAT 〈N3,N4>
MINIMUM RANGE (OCTETS)

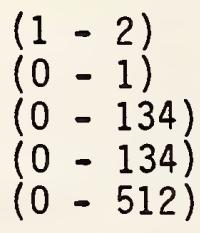

Note: The primitive data types and their maximum ranges for a specif ic file as described by the parameters above are maintained in the contents type file attribute. The contents type file attribute value is established at the file's creation and cannot be changed via FTAM for the life of the file. This implies that the data element types and ranges and data un it formats are fixed for all accessors of that file as long as the file exists.

An object identifier is a string of integers; FTAM document type parameterization will be achieved by exploiting that structure.

The final registration authority entity will be followed by a data un it description. The data un it description is a series of data element descriptions. Each data element description is an integer ident ical to the ASN.1 type code, followed by any required parameter values (as integers).

The following values correspond to the NBS primitives not found in ASN.1 and the integer value for ":" (separator).

$$
\begin{gathered}
\text { FLOAT }-127 \\
8859-126 \\
: \quad-125
\end{gathered}
$$


The following abstract syntax definition is given for the respresentation of float ing point numbers. The ability to transfer floating point numbers is not required. The only semantics associated with the float ing point primit ive type is its use to convey a value of relative precision.

The following notation is bel ieved to allow the movement of the same semantics as exist ing standards for float ing-point numbers (IEC 559 and IEEE 754) as well as represent an inf in ite number of binary floating point numbers.

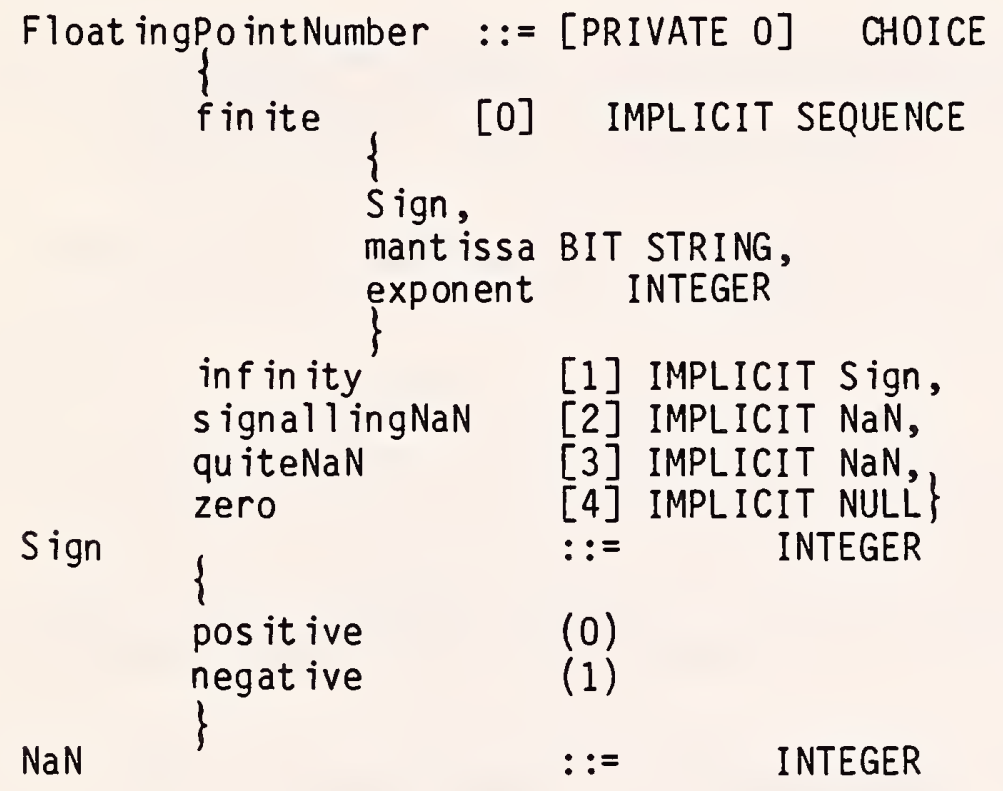

Notes: 1 . The mant issa is a number in the range $1 / 2 \leq$ mant issa $<1$.

2. Mant issa * 2 exponent.

3. The first bit in the mant issa is most sign if icant.

4. See IEEE 754 for definitions of terminology such as NaN.

\subsubsection{Character Sets}

The character sets IA5 and $8859 / 1$ have been spec if ied for use. The following describe how each of the character sets will be implemented.

\section{IA5}

The IA5 character set leaves 2 options and 10 characters unspecified. The following defin itions will be used.

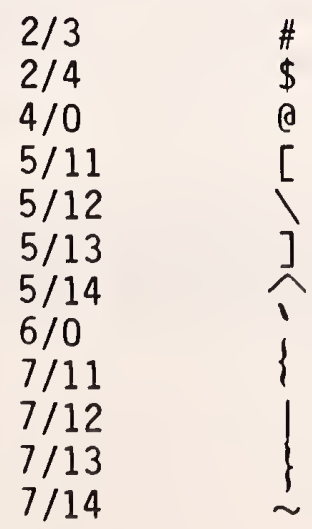


(Note this is exactly the International Reference Version (IRV) specified in the IA5 standard except that the code $2 / 4$ has the graph ic rendition " $\$$ " instead of the IRV-specified value of " $x$ ")

The following details how control characters should be handled.

a) The semantics of format effectors will be preserved.

b) Transmission control characters, device control characters, information separators, and "other" control characters will simply be preserved via the ir codes.

c) Code extension should not be used. If it is, the code extension characters should be preserved as in the case of the transmission control characters and any printing characters that form later parts of escape sequences will be interpreted as stand alone characters.

d) Combined horizontal and vertcal movement of cursor pos it ion ing will not be preserved.

2. $8859 / 1$

The Lat in Alphabet No. 1 will be used to specify the printable rendition of $\mathrm{CO}$ and $\mathrm{C} 1$.

The CO control characters and the ir associated rules will be taken from the IAS def in it ion.

The Cl control characters will simply have the ir codes preserved across a transfer.

\subsubsection{Document Type Negot iation Rules}

1. Connect ion Establ ishment:

DocumentTypeNames are to be negotiated by subset (of the proposed base DocumentTypeNames) without regard to DU syntax parameter(s) that may be supplied on any DocumentTypeNames which require a DU syntax specification.

\section{File Creation:}

An F-CREATErequest must conta in a DocumentTypeName from the negot iated set of base DocumentTypeNames. If the DocumentTypeName being used requires DU syntax parameters, then these parameters must be supplied. If the DocumentTypeName be ing used requires DU syntax parameters and none are provided on the F-CREATErequest, then the F-CREATErequest must be rejected.

\section{File Open ing:}

It is recommended that the $F$ OPENrequest use the Document TypeName form (with appropriate DU syntax parameters), when proposing a Contents Type, in preference to the Constraint Set Name and Abstract Syntax Name form. 
Similarly, an F OPENresponse should use the DocumentTypeName opt ion (with appropriate DU syntax parameters) in the Contents Type field. This will allow the receiving ent ity to use the DocumentTypeName attributed to the file instead of receiving a Constraint Set Name and Abstract Syntax Name pair which does not reflect the file information contained in the NBS document types.

NOTE: An F OPENresponse without a DocumentTypeName (but carrying the Constraint Set Name and Abstract Syntax name form may cause the in itiator to issue an F_CLOSErequest.

\subsubsection{Relationship Between DUS, DEs and Document Types}

"Abstract Syntax" is used to refer to the syntact ic informat ion which is architecturally passed between the Application and Presentation layers. The Abstract Syntax defines data element (DE) types. The DE types are not necessarily ASN.1 primitive types. Data types may be made up of other data types. Data Elements are not defined in terms of other data elements.

A data unit (DU) is a sequence of one or more data elements. Architecturally, entire, single DEs are passed into and out of the application process. In a real implementation, DUs may be passed.

In order to maintain DU boundaries during transfer, file structuring information must be passed (IS08571-FADU DEFINITIONS, FTAM Part 2 section 5.3.2). A data element is referred to as a File Contents Data Element in ISO8571-FADU DEFINITIONS.

Document types refer to aspects of local processing and storage. Document types describe:

1) structural relationship between DUs,

2) structure of DUs, called DU syntax, and

3) data element types found in the file.

Because document types have to do with local processing and storage, the DU syntax makes assertions about the syntax, and size of DUs (records) in storage. Parameters on the document types provide this information about the syntax and size of the DUs.

\subsubsection{F-CANCEL ACt ion}

When an F-CANCEL is sent or received, the following will occur:

- no more data will be sent

- check point numbers are removed

- state of the file will be implementation dependent. 


\subsubsection{Error Handl ing Agreements}

1. Diagnostic is mandatory only when the Action Result or State Result is not zero.

2. General catch-all diagnostic is discouraged.

3. Further details subfield is mandatory. Use of octet string is discouraged.

4. Use of F-P-ABORT for other than protocol errors and catastrophic situations is discouraged.

5. When returning an error status in a file management related diagnostic (i.e., F-READ-ATTRIBUTE-response or F-CHANGE-ATTRIBUTE-response), identify the erroneous attribute by using the first two characters of Diagnost ic further-details to hold a 2-digit number (encoded in IA5) from the F-READ-ATTRIBUTE-request attributes abstract syntax def in it ion (ISO/DIS 8571/4 section 20-4):

$\begin{array}{ll}00 & \text { Filename } \\ 01 & \text { Contents-Type } \\ 02 & \text { Storage Account } \\ 03 & \text { Date and Time of Creation } \\ 04 & \text { Date and Time of Last Mod if ication } \\ 05 & \text { Date and Time of Last Read Access } \\ 06 & \text { Date and Time of Last Attribute Mod if ication } \\ 07 & \text { Ident ity of Creator } \\ 08 & \text { Identity of Last Modifier } \\ 09 & \text { Identity of Last Reader } \\ 10 & \text { Identity of Last Attribute Modifier } \\ 11 & \text { File Availabil ity } \\ 12 & \text { Permitted Actions } \\ 13 & \text { Filesize } \\ 14 & \text { Future Filesize } \\ 15 & \text { Access Control } \\ 16 & \text { Encryption Name } \\ 17 & \text { Legal Qual ifications } \\ 18 & \text { Private Use }\end{array}$

\subsubsection{Concurrency}

The concurrency control used by default for the first accessor of a file is:

$\begin{array}{ll}\text { read } & \text { shared } \\ \text { insert } & \text { exclusive } \\ \text { replace } & \text { exclusive } \\ \text { extend } & \text { exclusive } \\ \text { erase } & \text { exclusive } \\ \text { rattr } & \text { shared } \\ \text { cattr } & \text { exclusive } \\ \text { del file } & \text { exclusive }\end{array}$

For subsequent file accessors, the requested access and process ing mode service parameters are checked against this, and access given only if the request is for operations that are shared. 


\subsubsection{Security}

1. Users may provide "user Id" (In it iator Identity) and "password" (filestore password). If the information is provided, the information will be sent to the Responder on the F-INITIALIZE.

2. Users may provide "access passwords". If the information is provided, the passwords will be sent to the Responder in the "access passwords" parameter.

3. It is the respons ibility of each local system to provide security of its own real file store.

4. The syntax of "user Id" and "password" (filestore password) is system-dependent.

5. Encrypt ion of passwords will not be done by FTAM.

6. "User Id" and "password" will represent 'account' information (this may be different from the 'account' parameter).

7. A user of the file service must be known by the Responder.

8. A commonly def ined "anonymous user" convention will be provided for all systems that choose to support this capability. Access available to that user is locally determined. The ID to be used is ANON. Any password should succeed.

9. Password support in FTAM is not required.

\subsubsection{Negot iation}

The guidel ines for negotiation in the following table have been agreed.

Table 10.2 FTAM negotiation rules

Service or Parameter

F-INITIAL IZE

Req.Pres.Con textMgmt

Req.Func. Un its

Req.Attr. Groups

Req.Comm.Qual ity

of services

Req. ContentTypel ist

\section{F-SELECT}

Attributes
Depends on or may be negot iated down by

Only by filename.

N.B. the filename on response and confirm must be that of an existing virtual file. 
Requested Access

\section{F-CREATE}

In it ial attributes

Requested Access

F-DELETE

F-READ-ATTR

F-CHANGE-ATTR

Attributes

F-OPEN

Processing Mode

Contents Type

Concurrency Control

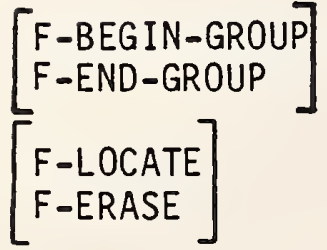

Negotiated by subset (in case of complete or partial success), must be cons istent with Functional Units negotiated, access control attribute and permitted actions attribute.

Cons istent with Functional Un its.

1) The attributes returned are with in the subset negotiated at in itial ization 2) The individual attribute values returned must be consistent with negotiation/ranges for that attribute. 3) The Responder returns values for all attributes which differ from the actual request.

as in F-SELECT

Cons istent with Functional Units.

Consistent with Functional Un its, service $c l$ ass and requested access.

as for F-READ-ATTR IBUTE

If any attribute cannot be successfully changed, then an error more severe than warning should be returned and no attribute should be changed.

Not Negotiated.

Must be cons istent with Functional Un it and Requested Access negotiated, with the permitted actions attribute and the contents type name.

Def ined in DIS.

More restrict ive than concurrency control of F-SELECT; cons istent with the concurrency control of other users.

Consistent with Functional Un its and service class.

Consistent with Functional Un its, requested access (and therefore also permitted actions). Service Class and Process ing Mode 


$$
\begin{aligned}
& {\left[\begin{array}{l}
\text { F-READ } \\
\text { F-WRITE }
\end{array}\right]} \\
& {\left[\begin{array}{l}
\text { F-DATA/F-DATA-END } \\
\text { F-CANCEL } \\
\text { F-TRANSFER-END }
\end{array}\right]}
\end{aligned}
$$

Functional Units, Service Class and number of bulk data transfers, Requested Access and Processing Mode.

Functional Un its and Service Class.

\subsubsection{Presentation Context Negot iation}

After successful negotiation of a presentation context between two applications, a presentation context, i.e. a pair of (abstract syntax, transfer syntax), has been agreed between the application and presentation entities. The transfer syntax may have a wider range than the associated abstract syntax, i.e., it may also encode data types not contained in that abstract syntax.

The understanding (for the Implementors Agreements) is that for that specific application association the transfer syntax is restricted to the abstract syntax. That means encodings of data types not contained in the negotiated abstract syntax may be rejected by the receiving presentation entity.

\subsubsection{Steps of Presentat ion Context Negot iat ion}

There are four entities involved in the negotiation of a Presentation Context, two Application Ent it ies, and two Presentation Ent ities. The relationship among the entities is as shown in the figure below:

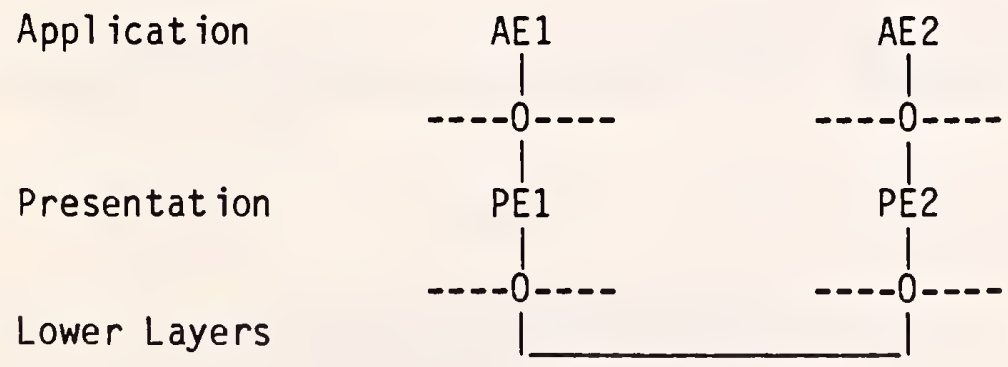

Figure 10.1 Relationship among ent it ies

AE1 - Application Ent ity 1 - needs an additional abstract syntax

AE2 - Applicat ion Entity 2

PE1 - Presentation Ent ity on AE1's node

PE2 - Presentation Entity on AE2's node 
Once $A E 1$ determines that it has a need to use an additional abstract syntax, there are seven steps in the negotiation of a Presentation Context.

They are:

1) AEl informs PEI that it needs a new abstract syntax, say AS1.

2) PEl picks a favorite transfer syntax, or set of transfer syntaxes, that can encode ASl, say TSl.

3) PE1 asks PE2 if it can deal with TS1. It does this by sending the pair (AS1,TS1) to PE2 along with an identifier for the Presentation Context.

4) PE2 decides if it can handle the transfer syntax TS1. If it can, it informs AE2 that there has been a request to use AS1.

5) AE2 decides if it can handle AS1, and informs PE2.

6) If PE2 has accepted TS1 and AE2 has accepted AS1 then PE2 informs PE1 that the Presentation Context has been accepted. If either TS1 or AS1 has been rejected, PE2 informs PE1 that the Presentation Context has been rejected.

7) PEI tells AE1 whether AS1 has been accepted or rejected.

\subsubsection{Conformance}

The FTAM SIG in cooperation with the COS FTAM Technical Committee will propose a revised conformance statement for the next version of these agreements.

\subsubsection{Migration Strategy}

NBS Phase 2 FTAM:

There is no backward compat ibility with NBS FTAM Phase 1. The following examples provide some of the technical clarifications as to why th is backward compatibility is impossible:

Phase 1

Uses Session directly

\section{Phase 2}

Uses CASE services

Uses Presentation Services

Filestore differences

PDU Abstract syntax differences

FADU Structuring abstract syntax differences

Transfer syntax differences

Transparency mechan ism differences

Service Class Negotiation differences

NBS FTAM Future Phases:

Given that FTAM Phase 2 is based on the forthcoming FTAM IS and that th is IS will provide the abil ity to pass "user version" information and will provide backward compatibility of protocol between versions of the IS, the workshop will specify mechan isms to provide FTAM product backward compat ibil ity for one previous product version (i.e., NBS Phase). 


\section{ISO PRESENTATION LAYER PROTOCOL}

Presentation Services are defined to meet the needs of many applications. The Presentation service is defined in ISO DIS 8822 and the Presentation protocol is defined in ISO DIS 8823. The general agreements about the implementation of Presentation are documented below.

\subsection{General}

1) Implementations will be based on the DIS (to be replaced by the IS) version of the ISO Presentation service and protocol.

2) A conformant implementation must be conformant to the ISO Presentation service and protocol, and must meet all of the requirements of this spec if icat ion.

\subsection{Functional Units}

1) The following functional un its are mandatory for implementation.

- Kernel

2) Implementation of any other functional un it is optional.

\subsection{Abstract Syntaxes}

1) The following abstract syntaxes must be supported.

- ISO 8571-FTAM

- ISO 8571-FADU

(FTAM PCI)

- ISO 8650-2-ACSE1

(File Structuring)

- NBS-AS1

(CASE PCI)

(Primitive data types)

2) The following abstract syntaxes are defined in these agreements, but are optional.

- NBS-AS2

- NBS-AS3

(float ing point numbers)

(file directories)

3) Any other abstract syntax may be supported.

4) Abstract syntaxes will be ident if ied by registered name.

\subsection{Transfer Syntaxes}

1) The following transfer syntaxes must be supported for all mandatory abstract syntaxes (also for NBS-AS2 and NBS-AS3 if implemented).

- NBS-TS1 
2) Any other transfer syntax may be implemented.

3) Transfer syntaxes will be identified by registered name.

12. COMMON APPLICATION SERVICE ELEMENTS PROTOCOL

Presently, the following agreements apply only when using FTAM.

CASE Part 2 Association Control Services are defined to meet the needs of many applications. The CASE Part 2 Association Control Service is defined in ISO DIS 8649/2 and the CASE Part 2 Association Control Protocol is def ined in ISO DIS $8650 / 2$. The general agreements about the implementation of CASE are documented below.

\subsection{General}

1) Implementations will be based on the DIS version of ISO $8649 / 2$ and ISO $8650 / 2$.

2) A conformant implementation must be ISO conformant as well as meet all of the requirements of this specification.

3) All services specified in ISO DIS $8649 / 2$ must be implemented.

\subsection{Application Contexts}

1) The following application contexts must be implemented. - ISO FTAM

\subsection{Application Entity Titles}

1) Application Entity Title will name a specific application entity on a spec if ic node (AE Instance).

2) An $A E$ Instance corresponds to exactly one application process.

3) The naming of application entities is external to this specificaion.

4) Application entity titles may have a many-to-one mapping to a PSAP address (i.e., al iases may be used).

5) An application entity title is a registered name of type OBJECT IDENTIFIER.

6) The application entity invocation does not have an address. 


\section{X.400 BASED MESSAGE HANDL ING SYSTEM}

\subsection{INTRODUCT ION}

This is an implementation agreement developed by the Implementor's Workshop sponsored by the U.S. National Bureau of Standards to promote the useful exchange of data between devices manufactured by different vendors. This agreement is based on, and employs protocols developed in accord with, the OSI Reference Model. While this agreement introduces no new protocols, it eliminates ambiguities in interpretations.

This is an implementation agreement for a Message Handling System (MHS) based on the X.400-series of Recommendations (1984) from the CCITT. Figure 13.1.1 displays the layered structure of this agreement.

This agreement can be used over any transport profile. In particular, this profile can be used over the transport protocol class 0 used over CCITT X.25, described in Section 7.2 of this document. In addition, this profile can be used over the transport profiles used in support of MAP (Manufacturing Automation Protocols) or TOP (Technical and Office Protocols). Note that the MAP or TOP environment must support the reduced Basic Activity Subset (BAS) as defined in X.410.

The UAs and MTAs require access to directory and routing services. A Directory Service is to be provided for each (vendor-specific) domain. Except insofar as they must be capable of providing addressing and routing described hereunder, these services and associated protocols are not described by this agreement.

The material on PRMD-PRMD message transfer in this implementation specification is intended to be stable enough to provide a reliable guide to implementation of X.400. The material on ADMD-PRMD and ADMD-ADMD is incomplete and serves as an indication of direction. 


\begin{tabular}{ll}
\hline User Agent Layer & CCITT $\times .420$ \\
\hline Message Transfer Agent Layer & CCITT $\times .411$ \\
\hline Reliable Transfer Service Layer & CCITT $\times .410$ \\
\hline Presentation Layer & CCITT $\times .410 \mathrm{sec} .4 .2$ \\
\hline Session Layer & CCITT $\times .225$ \\
\hline
\end{tabular}

Figure 13.1.1 The layered structure of this implementation agreement 


\subsection{SCOPE}

This agreement applies to Private Management Domains (PRMDs) and Administration Management Domains (ADMDS). Three boundary interfaces are specified:
A) PRMD to PRMD;
B) PRMD to ADMD;
C) ADMD to ADMD.

In case (A), the PRMDs do not make use of MHS services provided by an ADMD. In cases (B) and (C), UAs associated with an ADMD can be the source or destination for messages. Furthermore, in cases (B) and (C), an ADMD can serve as a relay between MDs. Figure 13.2.1 illustrates the interfaces to which the agreement applies.

$X .400$ protocols other than the Message Transfer Protocol (P1) and the. Interpersonal Messaging Protocol (P2) are beyond the scope of this agreement. Issues arising from the use of other protocols or relating to P1 components in support of other protocols are outside the scope of this document. This agreement describes the minimum level of services provided by Management Domains (MDs). Provision for the use of the remaining services defined in the $X .400$ Series of Recommendations is outside the scope of this document.

This agreement does not cover message exchange between communicating entities within a domain even if these entities communicate via P1 or P2. Bilateral agreements between domains may be implemented in addition to the requirements stated in this document. Conformance to this agreement requires the ability to exchange messages with conforming domains that have made no bilateral agreements. 
PRMD = Private Management Domain

ADMD = Administration Management Domain

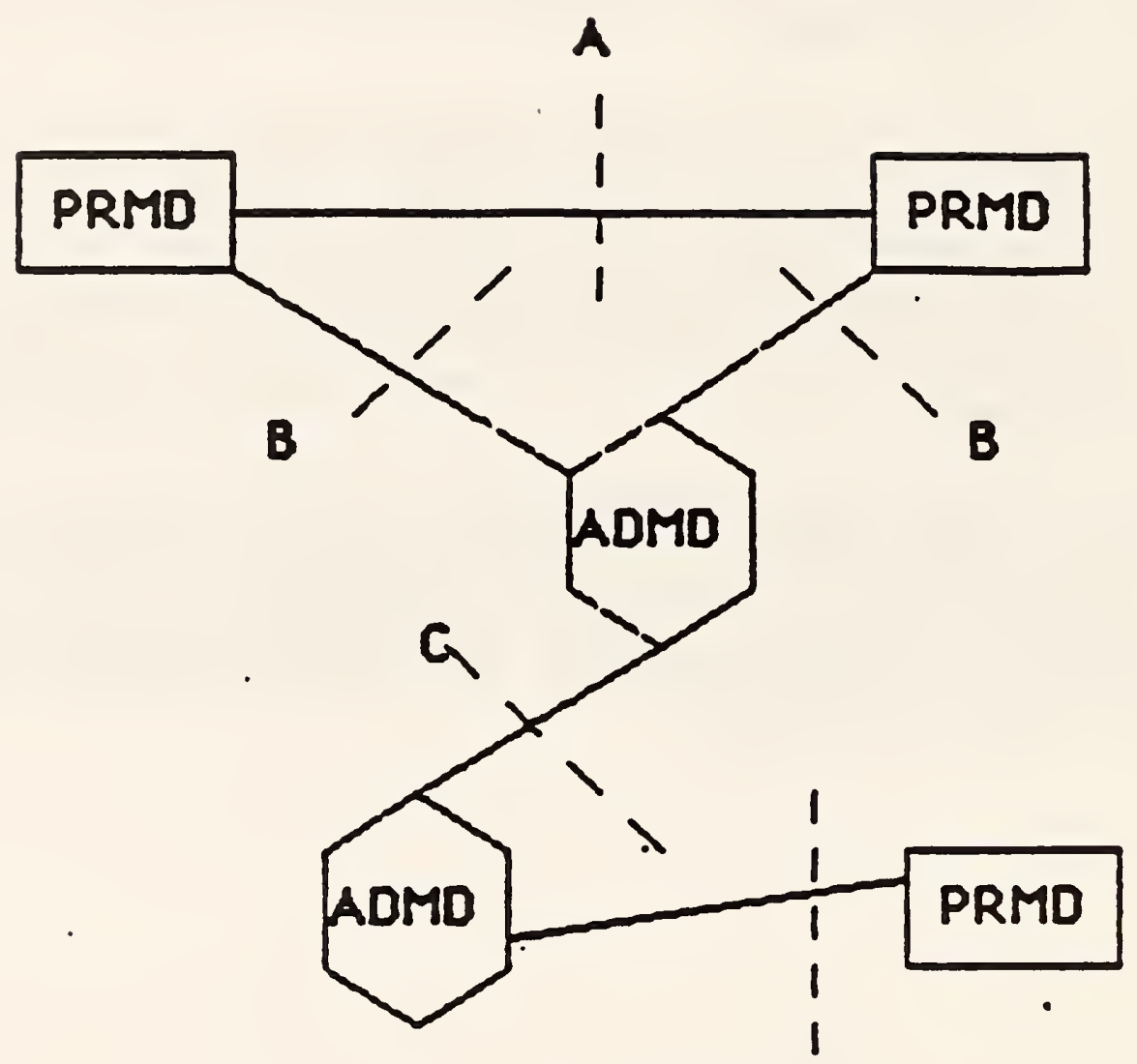

B

Figure 13.2.1 This agreement applies to the interface between:

(A) PRMD and PRMD; (B) PRMD and ADMD; (C) ADMD and ADMD 
This section is limited in scope to issues arising from the direct connection (interface A in Figure 13.2.1) of two PRMDs. "Direct" means that no ADMD provides MHS services to facilitate message interchange. "Direct" does not exclude those instances for which ADMDs provide lower layer services (e.g., X.25). Figure 13.3.1 schematically represents the scope of this section.

These issues relate to the use of the UAL (User Agent Layer) and MTL (Message Transfer Layer) services, protocol elements, recommended practices and constraints. In particular, this section addresses the P1 and P2 protocols and their related services in a direct connection environment. This section describes the minimum level of services provided by a PRMD. Provision for the use of the remaining services defined in the $X .400$ Series of Recommendations is beyond the scope of this section. 


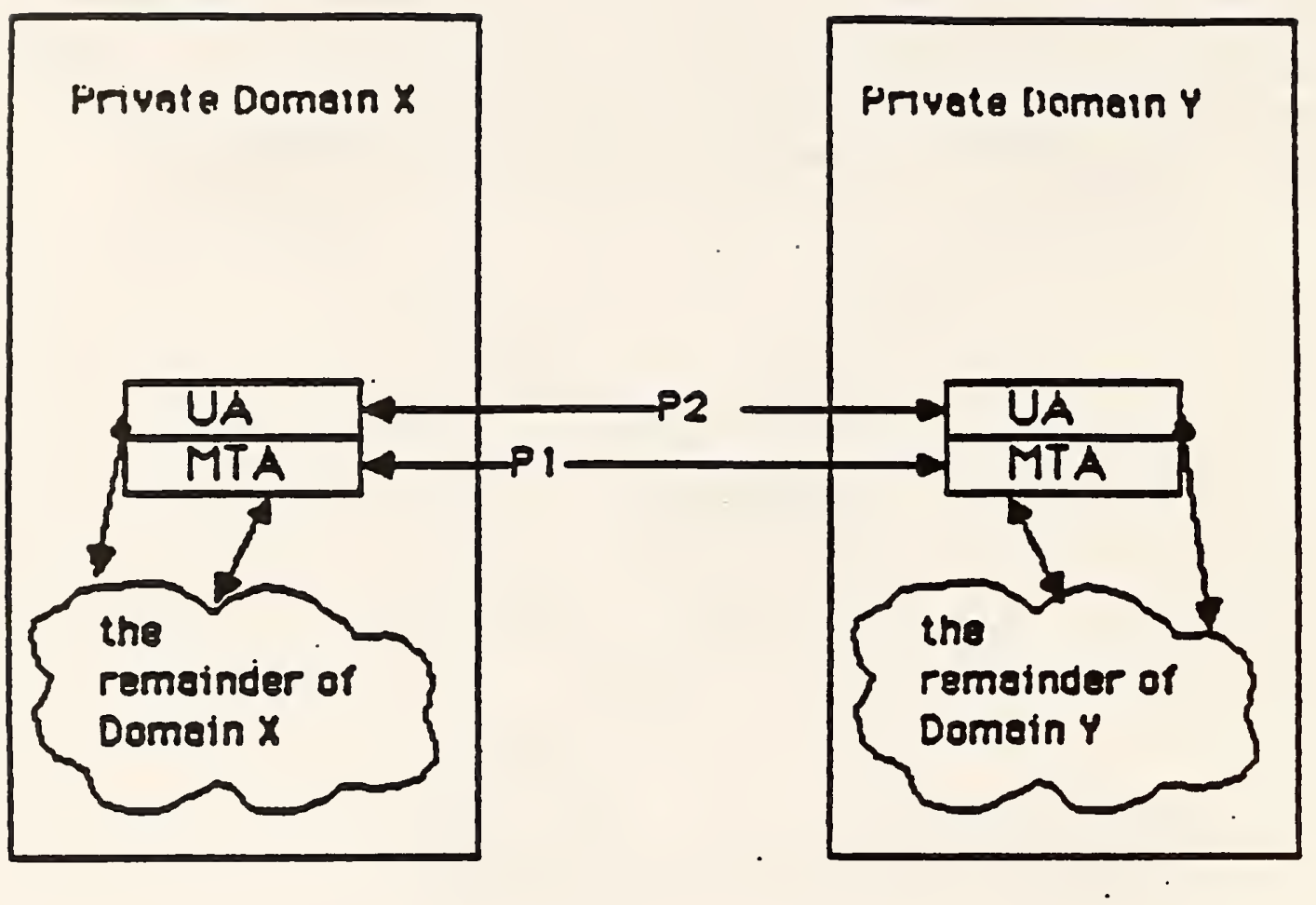

Figure 13.3.1 Interconnection of private domains 


\subsubsection{Service Elements and Optional User Facil it ies}

This section identifies those service elements and optional user facil it ies that must be provided in support of P1 and P2.

\subsubsection{Class if ication of Support for Services}

The classification of UA and MT-Service elements is used to define characteristics of equipment. Equipment can cla im SUPPORT or NON-SUPPORT of a Service; in the case of UA-service elements, a separate class if ication is given for Origination and Reception.

The service provider is defined as the entity providing the service, in this case, the MTL or the UAL. The service user is either the MS user or the UAL. The classification of provider and user relates to the sublayer for which the service element is defined.

\subsection{Support (S)}

This means that:

a. The service provider makes the service element available to the service user.

b. The service user gives adequate support to the MHS to invoke the service element or makes information associated with the service element available.

Support for Origination means that:

a. The service provider makes the service element available to the service user for invocation.

b. The service user gives adequate support to the end user of the MHS to invoke the service element.

Support for Reception means that the service provider makes information associated with the service element available to the service user.

Note: A UA- or MT-service element can carry information from originator to recipient only if:

- the service element is available to the or iginator,

- the service element is available to the recipient, and

- all intermediate steps carry the information. 


\subsection{Non-Support (N)}

This means that the service provider is not required to make the service element available to the service user. However, the service provider should not regard the occurrence of the corresponding protocol elements as an error and should be able to relay such elements. Implementations making a profile available should indicate deviations (additions or deletions) with respect to the requirement in the profile.

\subsection{Not Used (N/U)}

This means that al though the Recommendations allow this service element, this profile does not use it.

\subsection{Not Applicable (N/A)}

This means that this service element does not apply in this particular case (for originator or recipient).

\subsubsection{Summary of Supported Services}

a. Within a PRMD, a User Agent must support all P2 BASIC IPM Services $(X .400)$ and all P2 ESSENTIAL IPM Optional user facilities (X.401) subject to the qualifiers listed in APPENDIX A.

b. Within a PRMD, a MTA must support all BASIC MT Services (X.400) and al 1 ESSENT IAL MT optional user facilities $(X .401)$ subject to the qualifiers listed in APPENDIX A.

c. No support is required of the additional optional user facilities of X.401.

\subsubsection{MT Service Elements and Optional User Facilities}

Tables 13.3.1 through 13.3.3 show the message transfer (MT) service elements and optional user facilities. 
Table 13.3.1 Bas ic MT service elements

\begin{tabular}{ll}
\hline \multicolumn{1}{c}{ Service Elements } & \multicolumn{1}{c}{ Support (S) or } \\
Non-support (N)
\end{tabular}

a: Not applicable to co-res ident UA and MTA.

Table 13.3.2 MT optional user facil it ies

provided to the UA-selectable on a per-message bas is

\begin{tabular}{|c|c|c|}
\hline MT Optional User Facil it ies & Categorization & $\begin{array}{ll}\text { Support }(S) & \text { or } \\
\text { Non-support } & (\mathrm{N}\end{array}$ \\
\hline $\begin{array}{l}\text { Alternate Recipient Allowed } \\
\text { Convers ion Proh ib it ion } \\
\text { Deferred Del ivery } \\
\text { Deferred Del ivery Cancellat ion } \\
\text { Del ivery Not if icat ion } \\
\text { Disclosure of Other Rec ip ients } \\
\text { Expl ic it Convers ion } \\
\text { Grade of Del ivery Select ion } \\
\text { Mult i-dest inat ion Del ivery } \\
\text { Prevent ion of Non-del ivery Not if icat ion } \\
\text { Probe } \\
\text { Return of Contents }\end{array}$ & $\begin{array}{l}E \\
E \\
E \\
E \\
E \\
E \\
A \\
E \\
E \\
A \\
E \\
A\end{array}$ & 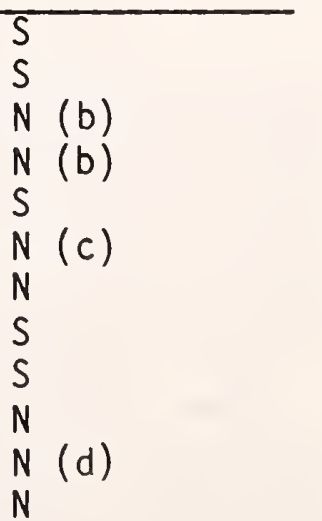 \\
\hline
\end{tabular}


Table 13.3.3 MT optional user facilities

provided to the UA agreed for a contractual period of $t$ ime

\begin{tabular}{lll}
\hline MT Opt ional User Facil ities & Categorization & $\begin{array}{l}\text { Support (S) or } \\
\text { Non-Support (N) }\end{array}$ \\
\hline Alternate Recipient Assignment & $A$ & $N$ \\
Hold for Del ivery & $A$ & $N / U$ \\
Impl ic it Conversion & $A$ & $N$ \\
\hline
\end{tabular}

E: Essential optional user facility.

A: Add it ional optional user facility.

b: A local facil ity subject to qual if iers in APPENDIX A.

c: Support not required for an originating MT user; support must be provided for recipient MT users.

d: Subject to qual if iers in APPENDIX $A$.

\subsubsection{IPM Service Elements and Optional User Facil it ies}

Tables 13.3.4 through 13.3.6 show the IPM service elements and opt ional user facil ities.

Table 13.3.4 Bas ic IPM service elements

\begin{tabular}{lll}
\hline \multicolumn{1}{c}{ Service Elements } & $\begin{array}{c}\text { Origination } \\
\text { by UAS }\end{array}$ & $\begin{array}{c}\text { Reception } \\
\text { by UAS }\end{array}$ \\
\hline Access Management & $\mathrm{N} / \mathrm{U}(\mathrm{a})$ & $\mathrm{N} / \mathrm{U}(\mathrm{a})$ \\
Content Type Indicat ion & $\mathrm{S}$ & $\mathrm{S}$ \\
Converted Ind icat ion & $\mathrm{N} / \mathrm{A}$ & $\mathrm{S}$ \\
Del ivery Time Stamp Indication & $\mathrm{N} / \mathrm{A}$ & $\mathrm{S}$ \\
Message Ident if icat ion & $\mathrm{S}$ & $\mathrm{N} / \mathrm{A}$ \\
Non-del ivery Not if icat ion & $\mathrm{S}$ & $\mathrm{S}$ \\
Original Encoded Informat ion & $\mathrm{S}$ & $\mathrm{N} / \mathrm{A} \mathrm{(a)}$ \\
Types Indicat ion & $\mathrm{N} / \mathrm{A}$ & $\mathrm{S}$ \\
Registered Encoded Information Types & $\mathrm{S}$ & $\mathrm{S}$ \\
Submiss ion Time Stamp Ind ication & $\mathrm{S}$ & $\mathrm{S}$ \\
IP-message Ident if icat ion & $\mathrm{S}$ & \\
Typed Body & & \\
\hline
\end{tabular}

(a) Does not apply to co-resident UA and MTA. 
Table 13.3.5 IPM optional facil ities agreed for a contractual period of $t$ ime

\begin{tabular}{lll}
\hline Service Elements & Categor izat ion & $\begin{array}{c}\text { Support (S) or } \\
\text { Non-Support (N) }\end{array}$ \\
\hline ATternate Recipient Ass ignment & $A$ & $N$ \\
Hold for Del ivery & $A$ & $N$ \\
Implicit Conversion & $A$ & $N$ \\
\hline
\end{tabular}


Table 13.3.6 IPM optional user facil ities selectable on a per-message bas is

\begin{tabular}{|c|c|c|}
\hline IPM Optional User Facil it ies & $\begin{array}{l}\text { Origination } \\
\text { by UAs }\end{array}$ & $\begin{array}{l}\text { Recept ion } \\
\text { by UAs }\end{array}$ \\
\hline $\begin{array}{l}\text { Alternate Recip ient Allowed } \\
\text { Authoriz ing Users Ind icat ion } \\
\text { Auto-forwarded Indicat ion } \\
\text { Bl ind Copy Recip ient Ind icat ion } \\
\text { Body Part Encrypt ion Ind icat ion } \\
\text { Convers ion Proh ib it ion } \\
\text { Cross-referencing Ind icat ion } \\
\text { Deferred Del ivery } \\
\text { Deferred Del ivery Cancellat ion } \\
\text { Del ivery Not if icat ion }\end{array}$ & $\begin{array}{l}A(N) \\
A(N) \\
A(N) \\
A(N) \\
A(N) \\
E(S) \\
A(N) \\
E(N)(f) \\
A(N / U)(f) \\
E(S)\end{array}$ & $\begin{array}{l}A(N) \\
E(S) \\
E(S) \\
E(S) \\
E(S) \\
E(S) \\
E(S) \\
\text { N/A } \\
\text { N/A } \\
\text { N/A }\end{array}$ \\
\hline $\begin{array}{l}\text { Disclosure of Other Recipients } \\
\text { Exp iry Date Indicat ion } \\
\text { Expl ic it Convers ion } \\
\text { Forwarded IP-message Ind icat ion } \\
\text { Grade of Del ivery Select ion } \\
\text { Importance Ind icat ion } \\
\text { Mult i-dest inat ion Del ivery } \\
\text { Mult i-part Body } \\
\text { Non-rece ipt Not if icat ion } \\
\text { Obsolet ing Ind icat ion }\end{array}$ & $\begin{array}{l}A(N) \\
A(N) \\
A(N) \\
A(N) \\
E(S) \\
A(N) \\
E(S) \\
A(N) \\
A(N) \\
A(N)\end{array}$ & $\begin{array}{l}E(S) \\
E(S) \\
N / A \\
E(S) \\
E(S) \\
E(S) \\
N / A \\
E(S) \\
A(N) \\
E(S)\end{array}$ \\
\hline $\begin{array}{l}\text { Originator Indication } \\
\text { Prevention of Non-del ivery Not if ication } \\
\text { Primary and Copy Recipients Ind ication } \\
\text { Probe } \\
\text { Rece ipt Not if ication } \\
\text { Reply Request Ind icat ion } \\
\text { Replying IP-message Indication } \\
\text { Return of Contents } \\
\text { Sens it iv ity Ind icat ion } \\
\text { Subject Indicat ion }\end{array}$ & $\begin{array}{l}E(S) \\
A(N) \\
E(S) \\
A(N) \\
A(N) \\
A(N) \\
E(S) \\
A(N) \\
A(N) \\
E(S)\end{array}$ & $\begin{array}{l}E(S) \\
N / A \\
E(S) \\
N / A \\
A(N) \\
E(S) \\
E(S) \\
N / A \\
E(S) \\
E(S)\end{array}$ \\
\hline
\end{tabular}

$f$ : A local facil ity subject to qual if iers in APPENDIX A. 


\subsection{2 $\times .400$ Protocol Def in it ions}

\subsubsection{Introduction}

This section reflects the agreements of the NBS/OSI Workshop regarding P1 and P2 protocol elements.

\subsection{Protocol Class if ication}

The protocol class if ications are defined:

a) UNSUPPORTED $=x$

These elements may be generated, but no specific processing should be expected in a relaying or del ivering doma in. A relaying doma in must at least relay the semantics of the element. The absence of these elements should not be assumed, in a relaying or del ivering domain, to convey any sign if icance.

b) SUPPORTED $=\mathrm{H}$

These elements may be generated. However, implementations are not required to be able to generate these elements. Appropriate actions shall be taken in a relay ing or del ivering doma in.

c) GENERATABLE $=G$

Implementations must be able to generate and handle these protocol elements, al though they are not necessarily present in all messages generated by implementations of this profile. Appropriate actions shall be taken in a relaying or delivering domain.

d) REQUIRED $=R$

Implementations of this profile must always generate this protocol element. However, its absence cannot be regarded as a protocol violation as other MHS implementations may not require this protocol element. Appropriate actions shall be taken in a relaying or del ivering doma in.

e) MANDATORY $=M$

This must occur in each message as per $X .411$ or $X .420$ as appropriate; absence is a protocol violation. Appropriate actions shall be taken in a relaying or del ivering doma in. 


\subsection{General Statements on Pragmatic Constraints}

a. Where a protocol element is defined as a choice of Numeric String and Printable String (i.e., Country Name, Administration Domain Name and Private Domain Identifier), then a numeric value encoded as a printable string is equivalent to the same value encoded as a numeric string.

b. The maximum number of recipients in a single MPDU is $32 \mathrm{~K}$ - 1 (that is, 32767). However, no individual limits on the number of occurrences (recipients) are placed on the following protocol elements: Authorizing Users, Primary Recipients, Copy Recipients, Blind Copy Recipients, nbsoletes and Cross References. Additionally, there is no limit on the number of Reply to Users. This is a local matter for the originating system.

c. Use of strings. A Printable String is defined in terms of the number of characters, which is the same number of octets. For T.61 strings the number of octets is twice the number of characters specified.

d. The ability to generate maximum size elements is not required, with the exception of the component fields in the Standard Attribute List, in which case it is required.

\subsection{MPDU Size}

The following agreements govern the size of MPDUs:

a. All MTAEs must support at least one MPDU of at least one megabyte.

b. The size of the largest MPDU supported by a UAE is a local matter. 
13.3.2.2 P1 Protocol Elements

13.3.2.2.1 P1 Envelope Protocol Elements

Table 13.3.7 contains Protocol Elements and their classes.

Table 13.3.7 P1 protocol elements

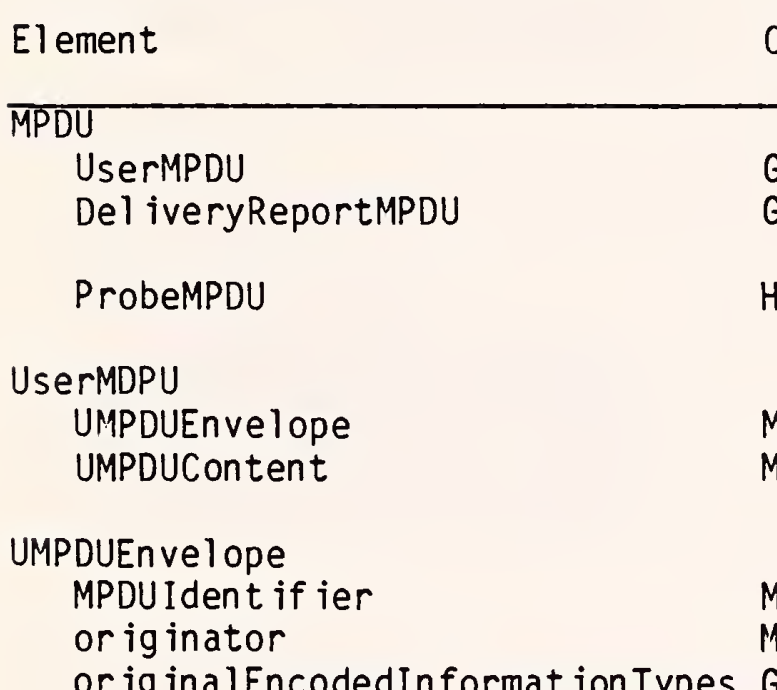

ContentType M

UAContentID $H$

Priority

PerMessageFlag

deferredDel ivery

PerDoma in $B$ il ateral Info

Rec ip ient In fo

Trace In format ion

UMPDUContent

MPDUIdent if ier

Globaldoma in Ident if ier

IA5String

PerMessageFlag

discloseRec ip ients

convers ionProh ib ited

alternateRec ip ient Al l owed

contentReturnRequest

PerDoma in B ilateral Info

CountryName

Admin istrat ionDoma in Name

Bilateral Info
Class

Restrictions and Comments
G

G

H

$M$

$M$

G

G

G

$X$

$X$

$M$

M

M

$M$

M

H

G

H

$X$

M

$M$

$M$
If th is field is absent, then the Encoded Information Type is "unspec if ied".

Maximum length $=16$ characters .

Max imum length $=2$ octets.

No 1 imit on number of occurrences Max imum number $=32 \mathrm{~K}-1$ occurr ences. More severe 1 imitations are by bilateral agreement.

Max imum length $=32$ characters, graphical subset only. Refer to T.50 for clarification of graphical subset.

Maximum length $=3$ characters . Maximum length $=16$ characters . Maximum depth $=8$; maximum length $=1024$ octets (including encoding). (cont inued on next page) 
Table 13.3.7 P1 protocol elements, Continued

\begin{tabular}{lc}
\hline Rec ip ientInfo & $M$ \\
recip ient & $M$ \\
Extens ion Ident if ier & $M$ \\
perRecip ientFlag & $X$ \\
Expl ic itConvers ion & \\
& \\
perRecipientFlag & $M$ \\
Respons ib il ityFlag & $M$ \\
ReportRequest & $M$ \\
UserReportRequest &
\end{tabular}

Trace In format ion

GlobalDoma in Ident if ier Doma inSuppl ied Info

Doma in Suppl ied Info
arrival
deferred
action
converted
previous

ORName

Encoded Informat ion Types

$b$ it string

M

$x$
$x$
$x$

TeletexNonBas icParameters

Presentat ionCapab il it ies

Del iveryReportMPDU

Del iveryReportEnvelope

Del iveryReportContent

Del iveryReportEnvelope

report

originator

Trace In format ion

$M$

M

Del iveryReportContent

or iginal

intermed iate

UAContent ID

ReportedRec ip ient Info

returned
$M$

$M$

$X$

M

$M$
$X$
$M$
$H$
$X$

$X$
$M$
$X$

Max imum value $=32 \mathrm{~K}-1(32767)$

Maximum length $=2$ octets.
Reference should be made to Version 3 of the X.400 Implementor's Guide for information related to Trace sequencing.
Del ivery can only occur if match is made with Registered Encoded Information Types. Individual vendors may impose 1 imits. Maximum length $=3$ octets.
Max imum number $=32 \mathrm{~K}-1$ occurrences. Can only be issued if specifically requested in the originating message. 
Table 13.3.7 P1 protocol elements, continued

Element
bill ing Information

ReportedRecip ient In fo
recip ient
Extens ions Ident if ier
PerRecip ientFlag
LastTrace Informat ion
intendedRec ip ient
Supplementary Informat ion

Class

Restriction and Comments

$x$

Maximum depth $=8$; maximum length $=1024$ octets (including encoding).

$M$

M

$M$

$M$

$H$

$X$

M

$\mathrm{H}$

M

converted

Report

Report

Del ivered Info

NonDel ivered Info

G

G

Del iveredinfo

del ivery

typeofUA

NonDel ivered Info

Reasoncode

Diagnost iccode

ProbeEnvelope

probe

originator

ContentType

UAContent ID

or iginal
$M$

H

R

R

Whenever possible, use a meaningful diagnostic code.

This element must be generated by PRMDs with a PRIVATE value.
Maximum length $=64$ characters . NOTE: This is subject to change. Value is pending ver if ication by the CCITT SG VIII or IX.

Generated if del ivery is reported Generated if failure to deliver is reported.

Max imum length = 16 characters . If this field is absent, then the Encoded Information Type is "unspec if ied". 
Table 13.3.7 P1 protocol elements, cont inued

TraceInformation (under Probe M

Envelope)

PerMessageflag

contentLength

PerDoma in $B$ il ateral Info

Rec ip ientInfo
G

H

$X$

M. Maximum number $=32 \mathrm{~K}-1$ occurrences.

--End of Defin it ions 
Only form 1 variant 1 O/R names are supported.

Table 13.3.8 conta ins ORName Protocol Elements.

Table 13.3.8 ORName protocol elements

Element

Class

Restrictions and Comments

ORName

StandardAttr ibutel ist

Doma inDef inedAtt $r$ ibutel ist

StandardAttributel ist (1)

CountryName

$R$

Adm in istrat ionDoma in Name (4) R

X.121Address

Term ina I ID

PrivateDoma inName (2)

Organ izat ionName (2)

Un iqueUAIdent if ier

Personal Name

$x$

$X$

G

G

$X$

G

Organ izationalun it (3)

G

Doma inDef inedAttributel ist (5)

type

value

$x$

$M$

M

Personal Name

surName

given Name

in it ials

generat ionQual if ier

G
$M$

$X$

$M$
$X$

$R$
$R$
$X$
$X$
$G$
$G$
$X$
$G$

As defined in X.411, Maximum

length $=3$ characters .

Maximum length $=16$ characters

or digits.

Maximum length $=15$ digits.

Maximum length $=24$ characters.

Maximum length $=16$ characters .

Maximum length $=64$ characters .

Maximum length $=32$ digits.

Maximum length of values of subelements $=64$ characters .

NOTE: The possibil ity that th is value may be reduced to 40 characters is for further study by the CCITT.

Maximum length $=32$ characters per occurrence. A maximum of four occurrences are allowed.

Maximum $=4$ occurrences. Maximum length $=8$ characters. Maximum length $=128$ characters .

Maximum length $=40$ characters . Maximum length $=16$ characters . Maximum length $=5$ characters; excluding surname in itial and punctuation and spaces. 


$\begin{array}{lll}\begin{array}{l}\text { GlobalDomainIdentifier } \\ \text { CountryName } \\ \text { AdministrationdomainName (4) }\end{array} & M & \begin{array}{l}\text { Maximum length }=3 \text { characters. } \\ \text { Maximum length }=16 \text { characters or } \\ \text { digits. }\end{array} \\ \begin{array}{l}\text { PrivateDomainIdentifier } \\ \text { R }\end{array} & \begin{array}{l}\text { Maximum length }=16 \text { characters or } \\ \text { digits. This element must be } \\ \text { generated by PRMDs. }\end{array} \\ \text {--End of Definitions-- } & \text { (Continued on next page) }\end{array}$


Table 13.3.8 ORName Protocol Elements, Cont inued

Notes:

(1) The following apply for comparison of the Standard Attributes of an $0 / R$ Name:

1) Lower case is interpreted as upper case (for IA5).

2) Mult iple spaces may be interpreted as a single space. Originat ing doma ins shall only transmit single sign if icant spaces. If mult iple spaces are transmitted, non-del ivery may occur.

(2) At least one of these must be supplied.

(3) These should be sent in ascending sequence, from the least sign if icant <0rganizational Unit> (lowest in organization hierarchy) to the most significant. Only those specified should be sent. (That is, an unspecified <0rganizational Un it> should not be sent along as a field of [null] content, nor zero length, etc.)

(4) This attribute shall contain one space in all ORNames of messages or iginated in a PRMD that is not connected to an ADMD, and in ORNames of recipients reachable only through a PRMD; otherwise, this attribute shall conta in an appropriate ADMD name.

(5) Many existing mail systems require attributes not present in these agreements. Doma in Defined Attributes are a method of providing these. Failure to support the specification of DDAs may prevent successful interworking with such existing mail systems unt il such time as all mail systems are capable of support ing del ivery via the standard attribute 1 ist only. Spec if ic recommendations on the use of DDAs are in the Recommended Practices section.

\subsubsection{P2 Protocol Profile (Based on $[X .420]$ )}

Tables 13.3.9 and 13.3.10 class ify the support for the P2 protocol elements required by this profile. The tables give restrictions and comment in add it ion to $[X .420]$.

Restriction on length is one of the types of restrictions. The reaction of implementation to a violation of this restriction is not defined by this profile. 


\subsection{P2 Protocol - Heading}

Table 13.3.9 below specifies the support for protocol elements in P2 Headings.

Table 13.3.9 P2 heading protocol elements

\begin{tabular}{|c|c|c|}
\hline Element & Class & Restrict ions and Comments \\
\hline \multicolumn{3}{|l|}{ UAPDU } \\
\hline IM-UAPDU & G & \\
\hline SR-UAPDU & $x$ & . \\
\hline \multicolumn{3}{|l|}{ IM-UAPDU } \\
\hline Head ing & $M$ & \\
\hline Body & $\ddot{M}$ & \\
\hline \multicolumn{3}{|l|}{ Head ing } \\
\hline IPMessage Id & M & \\
\hline or iginator & $R$ & \\
\hline author iz ingusers & $H$ & \\
\hline primaryRecipients & G & At least one of primaryRecipients, \\
\hline $\begin{array}{l}\text { copyRec ip ients } \\
\text { bl ind CopyRec ip ients }\end{array}$ & $\begin{array}{l}G \\
H\end{array}$ & $\begin{array}{l}\text { copyRec ip ients, or bl indCopyRec ip ients } \\
\text { must be present. }\end{array}$ \\
\hline $\begin{array}{l}\text { inReplyTo } \\
\text { obsol etes }\end{array}$ & G & \\
\hline crossReferences & $\mathrm{H}$ & \\
\hline subject & G & $\begin{array}{l}\text { Maximum length }=256 \text { octets; the } \\
\text { abil ity to generate the maximum size } \\
\text { subject is not required. }\end{array}$ \\
\hline expiryDate & $H$ & \\
\hline replyby & $H$ & \\
\hline replyTousers & $H$ & \\
\hline importance & $\mathrm{H}$ & Appropriate action is for further study \\
\hline sens it ivity & $\mathrm{H}$ & Appropriate action is for further study \\
\hline autof orwarded & $H$ & \\
\hline \multicolumn{3}{|l|}{ IPmessageId } \\
\hline ORName & $H$ & \\
\hline Printablestring & $M$ & Maximum length $=64$ characters. \\
\hline \multicolumn{3}{|l|}{ ORDescriptor } \\
\hline ORName & $H$ & $\begin{array}{l}\text { Specify the ORName whenever it is } \\
\text { poss ible. See APPENDIX B. }\end{array}$ \\
\hline free formName & $H$ & $\begin{array}{l}\text { Maximum length }=64 \text { characters, } \\
\text { graphical subset on ly ( } 128 \text { octets })\end{array}$ \\
\hline telephoneNumber & $x$ & $\begin{array}{l}\text { Maximum length }=32 \text { characters. Th is } \\
\text { allows for punctuation. It does not }\end{array}$ \\
\hline Recipient & $M$ & $\begin{array}{l}\text { take into account poss tole ruture } \\
\text { use by ISDN. }\end{array}$ \\
\hline ORDescr iptor & $M$ & \\
\hline reportRequest & $x$ & \\
\hline replyRequest & $H$ & \\
\hline
\end{tabular}


Table 13.3.9 P2 heading protocol elements, continued

\begin{tabular}{|c|c|c|}
\hline Element & Class & Restrictions and Comments \\
\hline $\begin{array}{l}\text { Body } \\
\text { BodyPart }\end{array}$ & G & $\begin{array}{l}\text { No } 1 \text { imit on number of BodyParts. } \\
\text { No } 1 \text { imit on length of any BodyPart } \\
\text { or the depth of ForwardedIPMessage } \\
\text { BodyParts nested. Class if ication is } \\
\text { subject to pending CCITT resolution. }\end{array}$ \\
\hline $\begin{array}{l}\text { SR-UAPDU } \\
\text { nonRece ipt } \\
\text { rece ipt } \\
\text { reported } \\
\text { actualRecip ient } \\
\text { intendedRec ip ient } \\
\text { converted }\end{array}$ & $\begin{array}{l}H \\
H \\
M \\
R \\
H \\
X\end{array}$ & \\
\hline $\begin{array}{l}\text { NonRece ipt In formation } \\
\text { reason } \\
\text { nonRece iptQual if ier } \\
\text { comments } \\
\text { returned }\end{array}$ & $\begin{array}{l}M \\
\text { M } \\
\text { H } \\
H\end{array}$ & $\begin{array}{l}\text { Maximum length }=256 \text { characters. } \\
\text { May only be issued if specifically } \\
\text { requested by originator. }\end{array}$ \\
\hline $\begin{array}{l}\text { Rece ipt In format ion } \\
\text { rece ipt } \\
\text { type } 0 \text { fRece ipt } \\
\text { Supplement ary In format ion }\end{array}$ & $\begin{array}{l}M \\
H \\
X\end{array}$ & $\begin{array}{l}\text { Maximum length }=64 \text { characters. } \\
\text { NOTE: This value is pending } \\
\text { verificat ion by the CCITT SG } \\
\text { VIII or IX. }\end{array}$ \\
\hline
\end{tabular}

\subsection{P2 Protocol - BodyParts}

All BodyParts with ident if iers in the range 0 up to and including $16 \mathrm{~K}-1$ are legal and should be relayed. BodyPart ident if iers corresponding to $X .121$ Country Codes should be interpreted as described in section 13.4.3.2.1. 


\subsection{Privately Defined BodyParts}

This section describes an interim means for ident ifying privately defined BodyParts. This subsection shall be replaced in a future ed it ion tak ing into account CCITT recommendations with equivalent functionality.

BodyPart : := CHOICE

[0] IMPLICIT IA5Text,

[1] IMPLICIT TLX,

$\dot{\bullet}$
[234]IMPLICIT UKBodyParts,

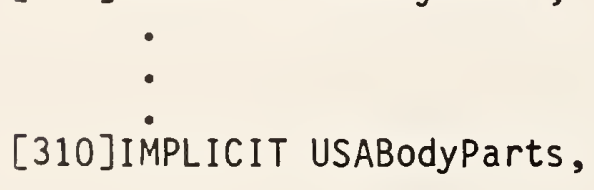

Where UKBodyParts and USABodyParts are defined as:

SEQUENCE BodyPartNumber, ANY

BodyPartNumber ::= INTEGER

In the Encoded InformationTypes of the P1 Envelope, the undefined bit must be set when a message contains a privately defined BodyPart. Each UA that expects such Bodyparts should include undefined in the set of del iverable Encoded Informat ion Types it registers with the MTA.

All BodyPartNumbers assigned must be interpreted relative to the BodyPart in which it is used, which is that tagged with the value [310] in the case of those defined with in the Un ited States. The NBS assigns un ique message BodyPartNumbers for privately defined formats with in the Un ited States.

Implementations are required to generate and image IA5Text.

Implementations should specify the other BodyPart types supported.

If an implementation supports a particular BodyPart type for reception, it should also be able to support that BodyPart type for reception if th is is part of a ForwardedipMessage.

For the BodyPart types currently considered, support for the protocol elements is as indicated in Table 13.3.10. 
13.3.2.3.2.2 P2 BodyPart Protocol Elements

Table 13.3.10 P2 BodyParts

\begin{tabular}{lc}
\hline Elements & $C$ \\
\hline BodyPart & \\
IA5Text & $G$ \\
TLX & $X$ \\
Voice & $X$ \\
G3Fax & $X$ \\
TIF0 & $X$ \\
TTX & $X$ \\
Videotex & $X$ \\
National lyDefined & $X$ \\
Encrypted & $X$ \\
ForwardedIPMessage & $H$ \\
SFD & $X$ \\
TIF1 & $X$ \\
& \\
IA5Text & $H$ \\
repertoire & \\
IA5String & $M$
\end{tabular}

Class

Restrictions and Comment

TLX

$G$
$X$
$X$
$X$
$X$
$X$
$X$
$X$
$H$
$X$
$X$

$H$
$M$

$M$

Voice

Set

Bitstring

$M$

G3F ax

number0fPages

G3NonBasicParameters

SEQUENCE (OF BIT STRING)

BIT STRING

G3NonBasicParameters

TIF0

T.73Document

T.73ProtocolElement

TTX

numbernfPages

telexCompatible

TeletexNonBasicParams

SEQUENCE (of T61string)

T61String

TeletexNonBasicParams

graphicCharacterSets

controlcharactersets

pageFormats

miscTerminalcapabilities

privateUse
For rendition of IA5Text see APPENDIX C.

For further study by CCITT.

For further study by CCITT.

See NOTE:

Support for individual elements is for further study.

See NOTE:

See NOTE: 
Table 13.3.10 P2 BodyParts, Cont inued

\begin{tabular}{|c|c|c|}
\hline Element & Class & Restrict ions and Comments \\
\hline $\begin{array}{l}\text { Videotex } \\
\text { SET } \\
\text { VideotexString }\end{array}$ & $M$ & For further study by CCITT \\
\hline $\begin{array}{l}\text { Nat ionallyDef ined } \\
\text { ANY }\end{array}$ & M & \\
\hline $\begin{array}{l}\text { Encrypted } \\
\text { SET } \\
\text { BIT STRING }\end{array}$ & $M$ & For further study by CCITT \\
\hline $\begin{array}{l}\text { Forwarded IPMessage } \\
\text { del ivery } \\
\text { Del ivery In format ion } \\
\text { IM-UAPDU }\end{array}$ & $\begin{array}{l}H \\
H \\
M\end{array}$ & \\
\hline $\begin{array}{l}\text { Del iveryInformation } \\
\text { ContentType } \\
\text { or iginator } \\
\text { original } \\
\text { Priority } \\
\text { Del iveryflags } \\
\text { otherRecipients } \\
\text { thisRecip ient } \\
\text { intendedRec ip ient } \\
\text { converted } \\
\text { submission }\end{array}$ & $\begin{array}{l}M \\
M \\
M \\
G \\
M \\
H \\
M \\
H \\
X \\
M\end{array}$ & \\
\hline $\begin{array}{l}\text { SFD } \\
\text { SFD. Document }\end{array}$ & $M$ & \\
\hline $\begin{array}{l}\text { TIF1 } \\
\text { T.73 Document }\end{array}$ & M & \\
\hline
\end{tabular}

Note: This element is not an addition to the def in it ion of the BodyPart. It is described here to show that the SEQUENCE may contain zero elements. A Problem Report has been submitted to the CCITT to clarify whether this is permissible. The NBS/OSI Workshop will adopt the CCITT decision. 


\subsubsection{Reliable Transfer Server (RTS)}

\subsubsection{Implementation Strategy}

Based on $\times .410$ clause 3 and $\times .411$ clause 3.5 .

\subsubsection{RTS option selection}

a) The maximum number of simultaneous associations is not limited in this profile; if the capacity of a system is exceeded, it should not initiate or accept additional associations.

b) Associations are established by the MTA which has messages to transfer.

c) Associations are released when they are not needed. Associations may also be ended prematurely due to internal problems of the RTS.

d) For both monologue and two way alternate associations, the initiator keeps the initial turn.

When establishing an RTS association, the following rules apply to the use of parameters in addition to those in $\mathrm{X} .410$ clause 3.2.1:

Dialogue mode:

Monologue must be supported for this profile; two-way alternate is used only if both partners agree.

Initial turn: Kept by the initiator of the association.

The 'priority-mechanism' and the 'transfer-time limit' are regarded as local matters. 


\subsubsection{RTS Protocol Options and Clarifications}

Realization of the RTS protocol is subject to the following rules in addition to those specified in $\times .410$ clause 4 :

a. One RTS association corresponds with one or more consecutive session connections (not concurrent ones). The first is opened with ConnectionData of type OPEN, and subsequent ones are opened with type RECOVER.

b. Recovery of Session connection is only by RTS initiator.

c. Checkpoint size:

- Checkpointing and No Checkpointing should be supported. Whenever possible, checkpointing should be used.

- The minimum checkpointsize is 1 (that is, 1024 octets).

d. Window size:

- Minimal value of 1 (if checkpointing is supported).

- WindowSize = 1 means: After an S-SYNCH-MINOR request is sent, wait until the confirmation is received before issuing an S-DATA, S-SYNCHMINOR, or S-ACTIVITY-END request.

e. APDUs should not be blocked into one activity.

f. Only one SSDU shall be transferred:

- Between two adjacent minor synch points.

- Between minor synch points and adjacent S-ACTIVITY-START and S-ACTIVITYEND requests.

- Between S-ACTIVITY-START and S-ACTIVITY-END without checkpoints.

g. A monologue association is defined as follows:

- The RTS user responsible for establishing the association is called the initiator.

- The initiator keeps the initial turn.

- APDUs are transferred in the direction of the initiator to the recipient only.

- There shall be no token passing.

- Only the initiator can effect an orderly release of the association.

h. A two-way alternate session is as described in X.410. 
i. In the UserData parameter of the S-U-ABORT, the ReflectedParameter will not be used in the Abort Information element.

$j$. When the S-ACTIVITY-RESUME is used to resume an act ivity in the same session connection as the one in which it started, this must happen immediately after the act ivity has been interrupted ( $i . e$. , no interven ing act ivity can occur). Otherwise, [X.410 clause 4.3 paragraph 1$]$ may be violated.

k. When S-ACTIVITY-RESUME is used to resume an activity started in another session connection, the following conditions must be met:

- The current session connection is of type "recover".

- The value of 0ldSessionConnect ionIdent if ier in S-ACTIVITY-RESUME must match the value of the Sessionconnect ionIdent if ier parameter used in the S-CONNECT of the prior session connection. This value is also ident ical to the SessionConnection Ident if ier in the ConnectionData (in PConnect, in SS-UserData) for the current session connection.

- This must occur as the first act ivity of the next session connection for the same RTS-association. It must be the first, otherwise [X.410 clause 4.5 .1 point 1] is violated.

Note: It is in the same RTS-ASSOCIATION because the use of S-ACTIVITYRESUME only makes sense with in the scope of one RTS association.

1. If the transfer of an APDU is interrupted before the confirmation of the first checkpoint, the value of the SynchronizationPointSerialNumber in S-ACTIVITY-RESUME should be zero.

The S-ACTIVITY-RESUME must be immediately followed by an S-ACTIVITYDISCARD.

m. In S-TOKEN-PLEASE, the UserData parameter shall contain an integer conforming to $X .409$ which conveys the priority.

n. The receiving RTS can use the value of the Reason parameter in the S-U-EXCEPTION-REPORT to suggest to the sending RTS that it should either interrupt or discard the current activity.

As stated in Version 3 of the $X .400$ Series Implementor's Guide, "On receipt of an 'unrecoverable procedure error' the current act ivity is not recoverable and the sending RTS issues an S-ACTIVITY-DISCARD. On receipt of any other reason code (including an undef ined value), the sending RTS issues an S-ACTIVITY-INTERRUPT followed by an S-ACTIVITYRESUME."

0. In the case of S-P-ABORT, the current activity (if any) is regarded as interrupted, rather than discarded. 
p. The following table illustrates the legal negotiation poss ib il it ies

allowed by $x .410$ clause 4.2 .1 regarding checkpoint size and window size:

Table 13.3.11 Checkpoint window size of IP

\begin{tabular}{|c|c|c|c|c|}
\hline & & \multirow{2}{*}{\multicolumn{3}{|c|}{ acceptor answer }} \\
\hline & & & & \\
\hline & & $\begin{array}{l}C S=0 \\
\text { (or unspec if ied) } \\
\text { WS unspec if ied }\end{array}$ & $\begin{array}{l}C S=m \\
W S=j \\
\text { or unspecif ied) }\end{array}$ & $\begin{array}{l}C S=n \\
W S=j \\
\text { (or unspec if ied) }\end{array}$ \\
\hline \multirow{2}{*}{$\begin{array}{l}\text { in it iator } \\
\text { proposal }\end{array}$} & $\begin{array}{l}C S=0 \\
\text { (or unspec if ied) } \\
W S=i \\
\text { (or unspecified) }\end{array}$ & legal & legal & legal \\
\hline & $\begin{array}{l}C S=k \\
W S=i \\
\text { (or unspec if ied) }\end{array}$ & legal & legal & not allowed \\
\hline
\end{tabular}

Legend:

- CS means CheckpointSize

- WS means WindowS ize

- $i, j, k, m$, and $n$ are integer values with the following relations:

$$
\begin{array}{ll}
0<m<k<n & \text { (values ass igned to CS) } \\
0<j<i & \text { (values assigned to WS) }
\end{array}
$$

- For unspecified parameters, the default applies. In th is case, the numeric relations apply, that is, the default values substitute for the unspecified integer. 


\subsubsection{RTS Protocol Limitations}

The RTS Protocol Limitations for this profile are listed in Table 13.3.12.

Table 13.3.12 RTS protocol elements

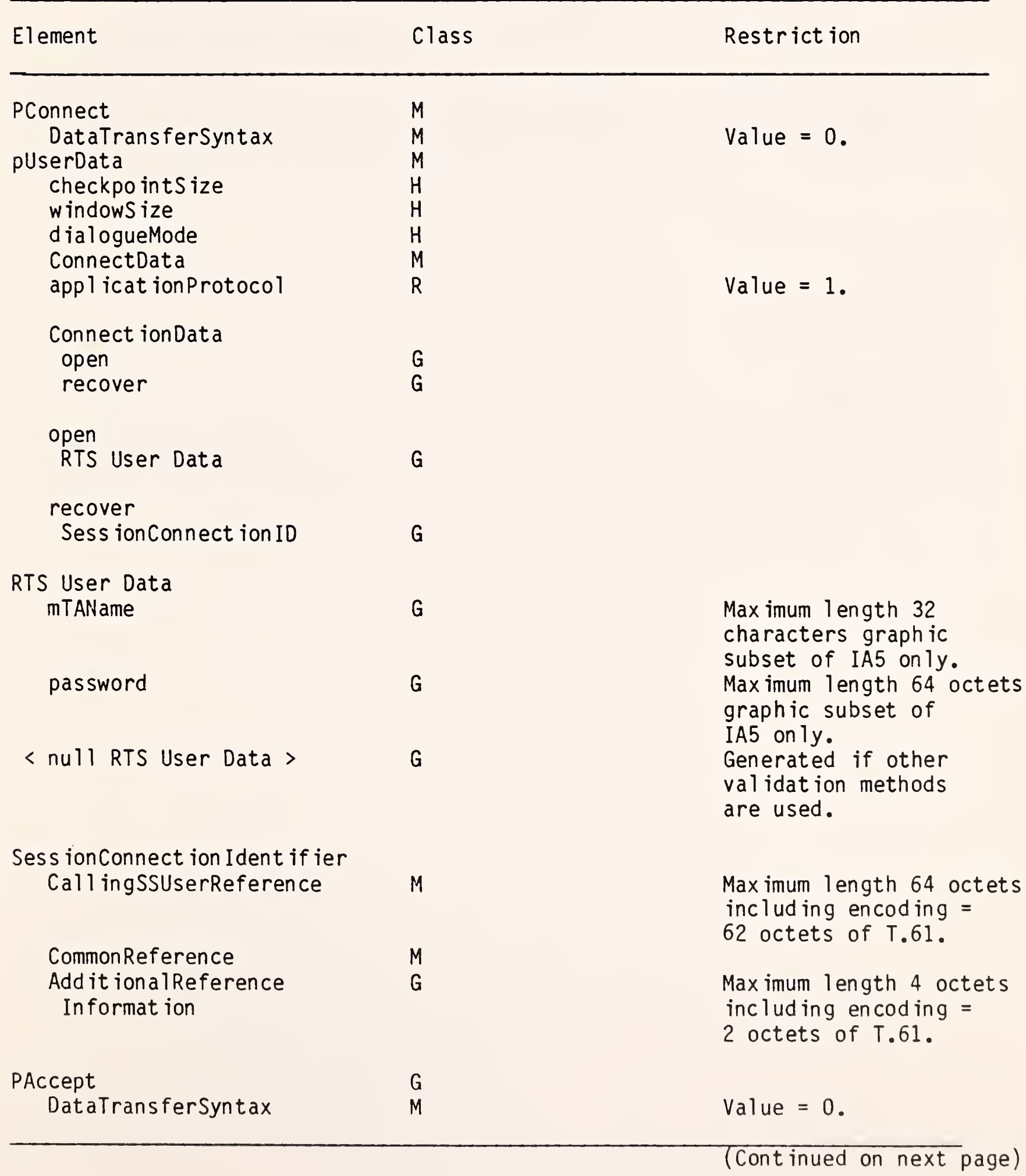


Table 13.3.12 RTS protocol elements, cont inued

\begin{tabular}{|c|c|c|}
\hline Element & Class & Restriction \\
\hline $\begin{array}{l}\text { PUserData } \\
\text { checkpo ints ize } \\
\text { windows ize } \\
\text { Connect ionData }\end{array}$ & $\begin{array}{l}M \\
H \\
H \\
G\end{array}$ & \\
\hline $\begin{array}{l}\text { PRefuse } \\
\text { RefuseReason }\end{array}$ & $G$ & \\
\hline $\begin{array}{l}\text { SS User Data } \\
\text { (in S-TOKEN-PLEASE) }\end{array}$ & G & \\
\hline $\begin{array}{l}\text { Abortinformation } \\
\text { ( in } S-U \text {-ABORT) } \\
\text { AbortReason } \\
\text { reflectedParameter }\end{array}$ & $\begin{array}{l}G \\
H \\
X\end{array}$ & Restricted to 8 bits \\
\hline
\end{tabular}




\subsubsection{Use of Session Services}

The session requirements and use of session are covered in section 8 of th is document.

\subsubsection{Data Transfer Syntax}

This section defines Presentation Transfer Syntax and notation rules applicable to these agreements. Implementations must conform EXACTLY as spec if ied in $X .409$ with no further restrictions. APPENDIX $C$ defines rendition of IA5 Text and T61 characters. 


\subsection{PRMD to ADMD and ADMD to ADMD}

\subsubsection{Introduction}

This section defines the implementation agreements that apply to the interface between two management doma ins when at least one is an ADMD. A message arriving at an ADMD has either no recipient with in that doma in or one or more recipients with in that domain. In the former case, the ADMD serves as a relay between two or more doma ins and the actions required of that ADMD are independent of the nature (PRMD or ADMD) of the doma ins. In the latter case, the ADMD is respons ible for del ivering messages to the proper recipient(s) with in its jurisdiction, and may also be responsible for relaying the message.

Given the two roles for an ADMD, this subsection describes two dist inct sets of functional requirements for an ADMD. The first is the relaying requirement that is needed to provide PRMD and other ADMD interworking. The second is analogous to the PRMD's support to its customers through the integrated UAs. These are distinct functional differences. The services provided to the UAs of an ADMD are independent of the requirement that an ADMD provide a function for interworking with any type of Management Doma in (MD). Figure 13.4.1 illustrates the two roles played by an ADMD.

This section is presented in the form of deviations from the agreements appl icable to PRMD-to-PRMD (section 13.3.0). Unless explicitly noted in the remainder of this section, all of the specifications for PRMD to PRMD apply to PRMD to ADMD and ADMD to ADMD. 


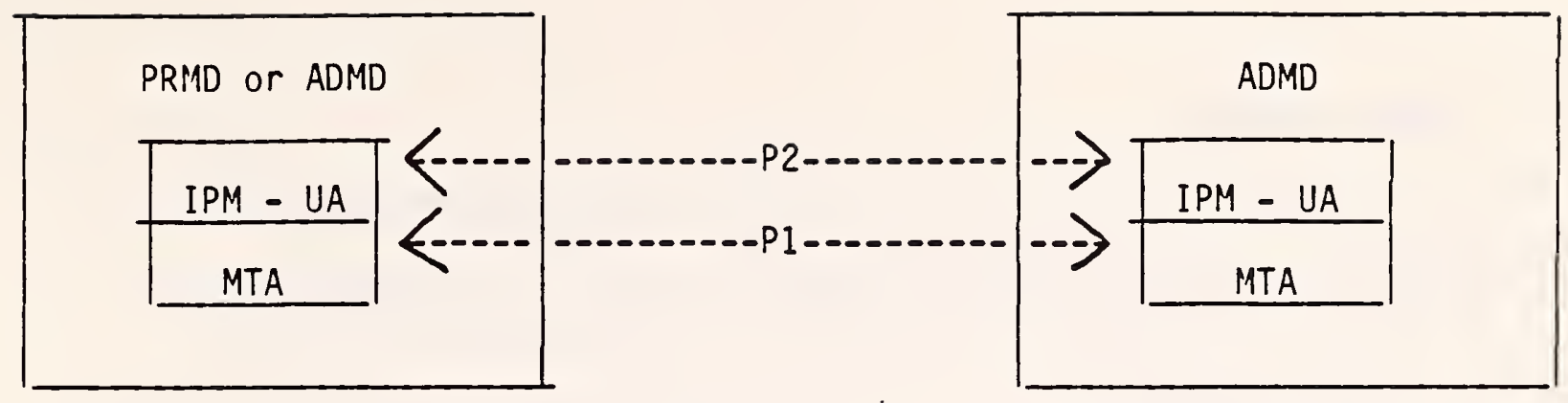

(a)

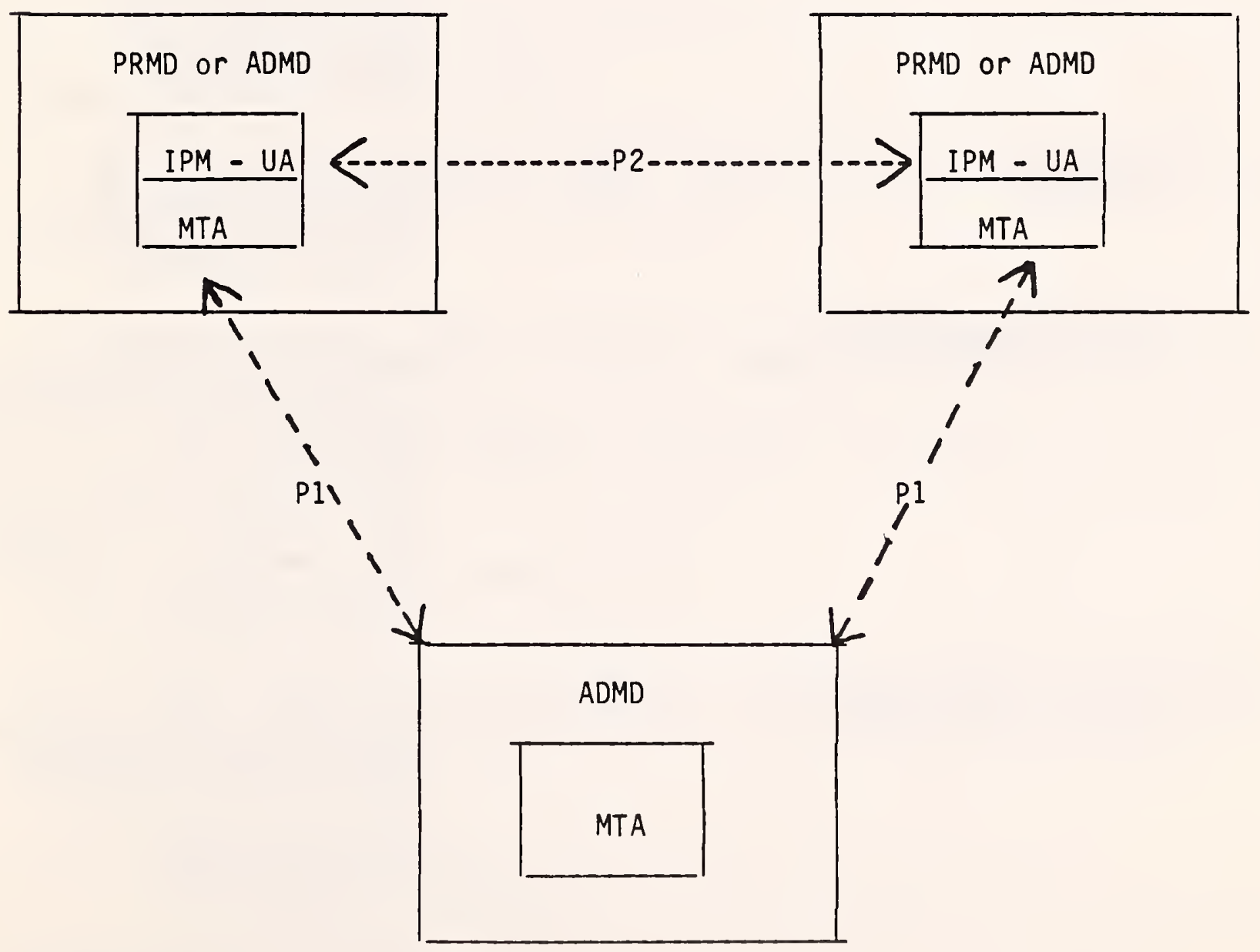

(b)

Figure 13.4.1 An ADMD may (b) or may not (a) serve as a relay. 


\subsubsection{ADMDs as Relays}

The following apply to an ADMD when it serves as a relaying domain.

1) ADMDs will relay all content types (not just P2) unchanged in the absence of a request for conversion.

2) P1 Protocol Change

\begin{tabular}{|c|c|c|}
\hline Protocol Elements & Class & \\
\hline
\end{tabular}

Del iveryReportContent

intermediate Trace Information G

If requested by other than the originating domain, return of the desired information cannot be assured, as the return path may be different and exclude that domain.

DeliveredInfo

type0fUA

H

H

ReportedRecipientinfo

Supplement ary Information

GlobalDomainIdentifier

PrivateDomainIdentifier
R
This element must be generated by PRMDs with value of "PRIVATE".

Domains providing access to TELEX/TELETEX recipients, whether directly or indirectly as a result of bilateral agreements between domains, must ensure that this information, when present, is accessible by the recipient of the delivery report.

For PRMDs. ADMDs shall take appropriate actions upon receipt of this element. 
3) $0 / R$ Names

0/R Names shall consist of:

CountryName

AdministrationDoma inName

as well as one of the following:

PrivateDomainName

Personal Name

OrganizationName

Organizationalunit

UniqueUAIdentifier

X121Address

and permits the optional inclusion of a

DomainDefinedAttributelist

Note that the destination PrivateDomainName or OrganizationName must be present if destined for a PRMD. The ADMD relaying the message to that destination PRMD requires this element.

P1 Originator Name

Management Domains (MDS) must specify in the ADMD name field of the $0 / R$ Name StandardAttributelist in P1, the name of the administration domain:

(a) to which the message is being sent (in recipient names)

(b) from which the message originated (in the originator name).

\subsubsection{Interworking with Integrated UAS}

If the message originates at a UA owned by an ADMD, or is delivered to such a UA, the 0/R Name follows the same Form 1 Variant 1 constraints as the base specifications; except that the ADMD name is the name of the owning ADMD and instead of supplying a PRMD Name, one (or more) of the following must be provided:

OrganizationName

Organizationalunit

PersonalName

and may optionally include a

DomainDefinedAttributelist 


\subsubsection{Differences with other Profiles}

\subsubsection{NTT Profile}

There are no outstanding issues regarding interworking between NTTconformant systems and NBS-conformant systems with the exception of the number of recipients. The Extension Identifier field may contain a maximum value of $32 \mathrm{~K}-1$; however, according to the current NTT profile, if a message with more than 256 recipients is received, the NTT-conformant domain will generate a nondelivery notification. This also applies to the ReportedRecipientinfo in a delivery report.

\subsubsection{CEPT Profile}

For further study.

\subsubsection{Connection of PRMDs to Multiple ADMDs.}

Given that Management Domain names (both PRMD and ADMD) shall be unique within the U.S., then when an ADMD is presented a message for transfer from a PRMD, it will accept 0/R Names (both originator and recipient) which have an AdministrationDomainName field value different than the administration's name. "Accept" implies the attempt to route/deliver the message shall be made, as appropriate, based upon the knowledge that MD names are unique.

Whether this functionality is required by an administration for conformance to this agreement is for further study.

If a PRMD is connected to two or more ADMDs which are not effectively connected (either directly or via a third ADMD), full $X_{.400}$ functionality shall not be available. Problems occur especially in the areas of:

-Naming

-Routing

-Replying.

\subsubsection{Connection of an ADMD to a Routing PRMD}

It is possible for a collection of interconnected private domains to establish one domain as the "gateway" to an ADMD, and hence to the world.

If an ADMD is connected to such a gateway PRMD, the individual private domains shall be registered with the administration. Administrations need not support such connections.

Note also that upon receipt by the ADMD of a message originating somewhere within the PRMD collection, that the TraceInformation may contain more than one element. 


\subsubsection{Management Domain Names}

All Management Domain Names (both Private and Administration) shall be unique within the U.S.

A central naming authority shall be established to register domain names.

\subsubsection{Envelope Validation Errors}

ADMDs will validate P1 Envelopes in the following areas:

a. The X.409 syntax of all elements should be checked.

b. The pragmatic constraint limits (lengths of fields and number of occurrances of fields) should be checked.

c. Semantic validation of the following selected set of elements should be done: originator O/R Name, original Encoded InformationTypes (but not against the actual contents), Priority, PerMessageflag, and Recipientinfo.

Validation of the MPDUIdentifier is for further study.

For relaying messages, Trace Information will be examined to detect looping route problems. Additional validation of the TraceInformation is for further study.

The actual Reasoncodes and Diagnosticcodes to be returned in the case of validation failure are for further study.

\subsubsection{For further study}

Among issues reserved for further study are:

1) RTS password management

2) Billing

3) Quality of service

4) Diagnostic information in support of operations

5) Intra-Domain Routing

6) Multi-Vendor Domains.

RTS password management is currently believed to be a local matter. 


\subsection{ERROR REPORTING}

This section describes appropriate actions to be taken by non-relaying domains upon receipt of protocol elements which are not supported in this profile, malformed MPDUs, unrecognized O/R Name forms, content errors, errors in reports, and unexpected values for protocol elements.

\subsubsection{MPDU Encoding}

The MPDU should have a context-specific tag of 0,1 , or 2 . If it does not have one of these tags, it is not possible to figure out who originated the message. Therefore, the way this error is reported is a local matter.

\subsubsection{Contents}

Once delivery to the UA has occurred, it is not possible to report errors in $\mathrm{P} 2$ information to the originator. In addition, it seems unreasonable to insist that the MTA that delivers a message ensures that the P2 content of the message is okay. As a result, the handling of content errors is a local matter.

\subsubsection{Envelope}

\subsubsection{Pragmatic Constraint Violations}

In all cases of pragmatic constraint violation, a nondelivery report should be generated with a Reasoncode of unableToTransfer, and a Diagnosticcode of invalidParameters. Note: it would be desirable for the CCITT to add a DiagnosticCode of pragmaticConstraintViolation to allow a more meaningful description of this problem. A request for this new DiagnosticCode has been submitted.

\subsubsection{Protocol Violations}

If not all required protocol elements are present, a nondelivery report with a Reasoncode of unableToTransfer and a DiagnosticCode of invalidParameters should be generated. Note: it would be desirable for the CCITT to add a DiagnosticCode of protocolviolation to allow a more meaningful description of this problem. A request for this new DiagonsticCode has been submitted.

If a protocol element is expected to be of one type, but is encoded as another, then a nondelivery report with a Reasoncode of unableToTransfer and a DiagnosticCode of invalidParameters should be generated. Note: it would be desirable for the CCITT to add a DiagnosticCode of protocolviolation to allow a more meaningful description of this problem. A request for this new DiagnosticCode has been submitted. 


\subsubsection{3 $0 / R$ Names}

The domain that has responsibility for delivering a message should also have the responsibility to send the nondelivery notification if the message cannot be delivered. Therefore, each domain should only validate the $0 / R$ Names of recipients with responsibility flags set to TRUE. In addition, a nondelivery notification can only be sent if the originator's $0 / R$ Name is valid.

If any element in the $0 / R$ Name is unrecognized or if the CountryName, AdministrationDomainName, and one of PrivateDomainName and OrganizationName are not all present, then a nondelivery report should be generated with a ReasonCode of unableToTransfer, and a DiagnosticCode of unrecognizedORName. If the message can be delivered even though the ORName is invalid, delivery is a local matter. Note, however, that if the message is delivered, the invalid ORName might be propagated through the $X .400$ system (e.g., by forwarding).

If the $0 / R$ Name has all of the appropriate protocol elements and the message still cannot be delivered to the recipient, the following DiagnosticCodes may appear in the nondelivery report: unrecognizedORName, ambiguousORName, and uaUnavailable.

\subsubsection{TraceInformation}

Since non-relaying domains need not do loop suppression, domains with responsibility for delivering the message need not be concerned about the semantics of the TraceInformation, that is, arrival time and converted EncodedInformationTypes can be provided to the UA without inspection by the MTAs of the domain as long as the TraceInformation is properly encoded according to $X .409$.

\subsubsection{Unsupported $X .400$ Protocol Elements}

The protocol elements defined in $X .400$ but unsupported by this profile are: the deferredDelivery and PerDomainBilateral Info parameters of the UMPDUEnvelope, the ExplicitConversion parameter of Recipient Info, and the alternateRecipientAllowed and contentReturnRequest bits of the PerMessageflag. Appropriate actions are described below for domains that do not support the protocol elements.

\subsubsection{1 deferredhelivery}

The domain shall do one of the following:

- deliver at once,

- hold for deferred delivery,

- return a nondelivery notification with a ReasonCode of unableToTransfer.

Note: it would be desirable for the CCITT to add a diagnostic code of noBilateralAgreement to allow a more meaningful description of this problem. A request for this new diagnostic code has been submitted. 


\subsection{PerDomainBilateralinfo}

If a domain receives this service element, the service element can be ignored, and the message should be delivered if possible.

\subsection{ExplicitConversion}

If ExplicitConversion is requested the message should be delivered if possible. That is, if the UA is registered to accept the EncodedInformationTypes of the message, then the message should be delivered even though the domain could not perform the requested conversion. If delivery is not possible, then a nondelivery report should be generated with a Reasoncode of conversionNotPerformed with no DiagnosticCode.

\subsubsection{4 alternateRecipientAllowed}

If a domain receives this service element the service element can be ignored, and this message should be delivered if possible.

\subsubsection{5 contentReturnRequest}

If a domain receives this service element, the service element can be ignored, and the message should be delivered if possible.

\subsubsection{Unexpected Values for INTEGER Protocol Elements}

There are three INTEGERs in the P1 Envelope. Appropriate actions are described below for domains receiving unexpected values for Priority, ExplicitConversion, and ContentType.

\subsection{Priority}

Additional values for Priority have been suggested by at least one group of implementors as upward compatible changes to the X.400 Recommendations. Therefore, if a domain receives an unexpected value for Priority, and this value is greater than one byte in length, a nondelivery report should be generated with a ReasonCode of unableToTransfer and DiagnosticCode of invalidparameters. If the value is less than or equal to one byte, the domain can either generate a nondelivery report as previously specified or default the Priority to normal and deliver the message.

\subsection{ExplicitConversion}

The message should be delivered if possible. That is, if the UA is registered to accept the EncodedInformationTypes of the message, then the message should be delivered even though the domain could not perform the requested conversion. If delivery is not possible, then a nondelivery. report should be generated with a ReasonCode of conversionNotPerformed with no DiagnosticCode. 


\subsection{ContentType}

If the ContentType is not supported, then a nondelivery report should be generated with a ReasonCode of unableToTransfer, and a DiagnosticCode of invalidParameters. Note: it would be desirable for the CCITT to add a DiagnosticCode of contentNotSupported to allow a more meaningful description of this problem. A request for this new DiagnosticCode has been submitted.

\subsubsection{Additional Service Elements}

In the absence of bilateral agreements to the contrary, receipt of privately tagged elements and protocol elements in addition to those defined in $\times .400$ will result in a nondelivery report with a Reasoncode of unableToTransfer and a DiagnosticCode of invalidParameters.

\subsubsection{Reports}

There is no mechanism for returning a delivery or status report due to errors in the report itself. Therefore the handing of errors in reports is a local matter.

\subsection{MHS USE OF DIRECTORY SERVICES}

Recommendation $X .400$ recognizes the need of MHS users for a number of directory service elements. Directory service elements are intended to assist users and their UAs in obtaining information to be used in submitting messages for delivery by the MTS. The MTS may also use directory service elements to obtain information to be used in routing messages. Some functional requirements of directories have been identified and are listed below.

1. Verify the existence of an $0 / R$ name.

2. Return the $0 / R$ address that corresponds to the $0 / R$ name presented.

3. Determine whether the $0 / R$ name presented denotes a user or a distribution list.

4. Return a list of the members of a distribution list.

5. When given a partial name return a list of $0 / R$ name possibilities.

6. Allow users to scan directory entries.

7. Allow users to scan directory entries selectively.

8. Return the capabilities of the entity referred to by the $0 / R$ name.

9. Drovide maintenance functions to keep the directory up-to-date. 
In addition to functionality, a number of operational aspects must be considered. These include user-friendliness, flexibility, availability, expandabil ity, and reliability.

Currently, these aspects of directory service elements and procedures are under study by both the CCITT and the ISO. Both organizations are committed to the development of a single Directory Service specification for use by MHS and all other OSI based applications.

Given the incomplete nature of the ongoing activities with in the CCITT and the ISO, no implementation details will now be provided for MHS use of Directory Services. Implementation agreements for MHS Use of Directory Services will be issued when current act ivities with in the CCITT and the ISO are stable.

It is recognized that these agreements enable a wide variety of naming and addressing attributes (see section 13.3.5.3.3 ORName Protocol Elements) where in each PRMD may adopt particular routing schemes $w$ ith in its doma in. These agreements make no attempt to recommend a standard pract ice for electron ic mail addressing.

Inter-PRMD addressing may be secured according to practices outs ide the scope of these agreements, such as:

- manual directories

- on-line directories

- ORName address specifications

- ORName address translation

Further, each PRMD may adopt naming and addressing schemes where in the user view may take a form entirely different from the attributes reflected in section 13.3.4.2.2 herein. And, each PRMD may have one user view for the originator form and another for the recipient form, and perhaps other forms of user addressing. In some cases (e.g., receipt not ifocation) these user forms must be preserved with in the constraints of these implementation agreements. However, mapping between one PRMD user form to another PRMD user form, via the $X .400$ ORName attributes of these agreements, is outside the scope of these agreements.

\subsection{CONFORMANCE}

In order to ensure that products conform to these implementation agreements, it is necessary to define the types and degrees of conformance testing products that must pass before they may be class ified as conformant. Th is section def ines the conformance requirements and provides guidelines for the interpretation of the results from this type of testing. 
In order to achieve a minimum level of confidence in the conformance of a product, the most basic requirements a product must meet to be classified as conformant to these agreements are provided. This minimal set was defined to ensure that the resulting MHS network will provide a reasonable interpersonal messaging facility to its users while still giving a reasonable assurance that conforming products can soon be made available. In addition, the full conformance requirements for products implement ing all aspects of $X .400$ Messaging governed by these agreements are provided.

This section is incomplete and will be enhanced in future versions of this agreement. Later versions will reflect the problems of conformance testing and will outl ine spec if ic practices and recommendations to aid the development of conformance tests and procedures.

\subsubsection{Defin ition of Conformance}

For this section, the term conformance is defined by the following:

1. The tests indicated for this section are intended to establish a high degree of confidence in a statement that the implementation under test (IUT) conforms (or does not conform) to the agreements of this section.

2. Conformance to a service element means that the information associated with the service element is made accessible to the user (person or process) whenever this agreement says that this information should be available.

Accessible means that information must be provided describing how a user (person or process):
a) causes appropriate information to be displayed, or
b) causes appropriate information to be obtained.

3. Conformance to P1, P2, and RTS as part of an X.400 OSI application requires that only the external behavior of that OSI system adheres to the relevant protocol standards.

In order to achieve conformance to this section, it is not required that the inter-layer interfaces be available for testing purposes.

4. Conformance to the protocols requires:
a) that MPDUs correspond to instances of syntactically correct data un its,
b) MPDUs in which the data present in the fields and the presence (or absence) of those fields is valid in type and semantics as defined in $X .400$, as qual if ied by this profile,
c) correct sequences of protocol data un its in responses (resulting from protocol procedures).


5. Statements regarding the conformance of any one implementation to this profile are not complete unless a Protocol Implementation Conformance Statement (PICS) is supplied.

6. The term "Implementation Under Test" (IUT) is interchangeable with the term "system" in the definition of conformance, and may refer to:

a) a domain, which may be one or more MTA's with co-located or remote UA's,

b) a single instance of an MTA and co-located UA with X.400 (P1, P2, RTS and session) software,

c) a relaying product with P1, RTS and session software,

d) a gateway product.

7. Tests for conformance apply independently to:
a) origination,
b) reception,
c) relaying.

\subsubsection{Conformance Requirements}

Conformance to this specification requires that all the services listed as supported in sections 13.3 and 13.4 of these agreements are supported in the manner defined, in either the CCITT X.400 Recommendations or these agreements.

It is the intention to adopt, where and when appropriate the testing methodology and/or the abstract test scenarios currently being defined by the CCITT X.400 Conformance Group. However it is recognized that formal CCITT Recommendations relating to $x .400$ Conformance Testing will not be available until 1988.

\subsubsection{In itial Conformance}

Th is section is intended to provide guidel ines to vendors who envisage having $X .400$ products available prior to any formal mechan ism, or 'Conformance Test Center' being made accessible that would allow for conformance to this product specification to be tested. 
It is feasible that vendors and carriers will want to enter bilateral test agreements that will allow for in itial trials to be carried out for the purposes of testing in itial interworking capabilities. It is equally feasible that for the purposes of testing interoperability, only a subset of this specification will in itially be tested. Therefore it is recommended that the following subset of total information be made accessible to allow for meaningful testing.

Note: By claiming conformance to this subset of information the vendor or carrier cannot claim conformance to this entire specification.

There are two aspects to the requirements, interworking and service, as described in the following sections.

\subsection{Interworking}

The interworking requirements for conformance impl ies that tests be done to check for the syntax and semantics of protocol data elements for a system as defined by their classifications (i.e., X, H, G, R, and M). For an origination system, this implies that the protocol elements generated must be correct. For a relay system, the correct protocol elements should be relayed as appropriate. And for a recipient system, a message with correct protocol elements must not be rejected where appropriate.

\subsection{Service}

For information available to the recipients $v i a$ the IPMessage Heading and Body, the following should be made accessible:

- IPMessage ID - only the Printablestring portion of the IPMessageId needs to be access ible.

- subject

- primaryRec ip ients

- copyRecipients

- bl indCopyRec ip ients

- author iz ingUsers

- or ig inator

- inReplyTo

- replyTousers

- importance

- sensit iv ity

- IA5Text BodyPart 


\section{DIRECTORY SERVICES PROTOCOLS}

The directory services protocols' implementation specifications are being prepared by the DS SIG.

\section{PERFORMANCE}

To be completed.

16. SECURITY

To be completed. 
$\underline{\text { NBS }}$

1. FIPS 107, Local Area Networks: Baseband Carrier Sense Multiple Access with Collision Detection Access Method and Physical Layer Specifications and Link Layer Protocol, NTIS, U.S. Department of Commerce, 5285 Port Royal Road, Springfield, VA 22161.

2. FIPS 100, Interface Between Data Terminal Equipment (DTE) and Data Circuit-Terminating Equipment (DCE) For Operation With Packet-Switched Data Communications Networks, NTIS, U.S. Department of Commerce, 5285 Port Royal Road, Springfield, VA 22161.

3. ICST/SNA-85-10, Implementation Agreements Among Participants of OSINET, edited by Jerry Mulvenna, National Bureau of Standards.

IEEE

1. IEEE Project 802

Local Area Network Standards P802.2 Logical Link Control November, 1982.

2. IEEE Project 802

Local Area Network Standards

IEEE Standard 802.4

Token - Passing Bus Access Method and

Physical Layer Specification.

3. IEEE Project 802

Local Area Network Standards

IEEE Standard 802.3

CSMA/CD Access Method and

Physical Layer Specification.

The above documents may be obtained from: IEEE Standards office, 345 East 47th Street, New York, N.Y. 10017.

$\underline{\text { ISO }}$

1. Addendum to DIS 8473 Covering Provision of the Connectionless-Mode Subnetwork Service, ISO/TC97/SC 6/N3453.

2. Network Service Definition, DIS 8348, ISO/TC97/SC6 N2990.

3. Addendum to the Network Service Definition Covering Connectionless Data Transmission, DIS 8348 DAD1, N3152. 
4. Addendum to the Network Service Defin it ion Covering Network Layer Addressing, DP 8348 DAD2, N3134.

5. Internal Organization of the NetworkLayer, WD, N3141.

6. Protocol for Providing the Connectionless Network Service, DIS 8473, N3154.

7. Information Processing Systems - Open Systems Interconnection Transport Service Def in it ion, ISO IS8072, 1984.

8. Information Processing Systems - Open Systems Interconnection Transport Protocol Spec if ication, ISO IS8073, 1984.

9. Information Process ing Systems - Open Systems Interconnection Session Service Def in ition, ISO DIS8326, 1984.

10. Information Process ing Systems - Open Systems Interconnection Session Protocol Specification, ISO DIS8327, 1984.

11. Information Process ing Systems - Open Systems Interconnection - File Transfer Access and Management Part 1: General Description, ISO DP8571/1, TC97/SC16 N 1669, February 1984.

12. Information Process ing Systems - Open Systems Interconnection - File Transfer Access and Management Part II: The Virtual Filestore, ISO DP $8571 / 2$, TC97/SC16 N1670, February 1984.

13. Information Process ing Systems - Open Systems Interconnection - File Transfer Access and Management Part III: Service Def in it ion, ISO DP8571/3, TC97/SC16 N1671, February 1984.

14. Information Process ing Systems - Open Systems Interconnect ion - File Transfer Access and Management Part IV: Protocol Specification, ISO DP8571/4, TC97/SC16 N1672, February 1984.

15. Data Commun ication - X.25 Packet Layer Spec if icat ion for Data Terminal Equipment, ISO/TC 97/SC 6 N 2641, ISO/DP 8208, 1983.

16. 7-b it Coded Character Set for Information Processing Interchange, ISO-646, 1973.

17. Information Interchange--Representation of Local Time Differentials, ISO-3307, 1975.

18. Draft Network Layer Management Protocol for the exchange of rout ing information between end systems and intermediate systems ISO/TC97/SC6/3862 January 1986.

19. Information Processing Systems - Open Systems Interconnection - File Transfer, Access and Management Part I: General Description, ISO DIS8571/1, TC97/SC21 N2371, August 1986.

20. Information Process ing Systems - Open Systems Interconnection - File Transfer, Access and Management Part II: The Virtual Filestore, ISO DIS8571/2, TC97/SC21 N2372, August 1986. 
21. Information Processing Systems - Open Systems Interconnection - File Transfer, Access and Management Part III: File Service Def in it ion, ISO DIS8571/3, TC97/SC21 N2373, August 1986.

22. Informat ion Processing Systems - Open Systems Interconnect ion - File Transfer, Access and management Part IV: File Protocol Specification, ISO DIS8571/4, TC97/SC21 N2374, August 1986.

23. Information Processing Systems - Open Systems Interconnect ion Connection-Oriented Presentation Service Definition, ISO DIS8822, TC97/SC21 N1594, May 1986.

24. Information Processing Systems - Open Systems Interconnection Connection-0riented Presention Protocol Spec if ication, ISO DIS8823, TC97/SC21 N1594, May 1986.

25. Information Processing Systems - Open Systems Interconnection Service Defin it ion for Common Appl ication Service Elements - Part 2: Association Control, ISO DIS8649/2, TC97/SC21 N1493, May 1986.

26. Information Process ing Systems - Open Systems Interconnection Protocol Specification for Common Application Service Elements Part 2: Association Control, ISO DIS8650/2, TC97/SC21 N1494, May 1986.

The above documents may be obtained from:

Frances E. Schrotter ANSI

ISO TC97/SC6 Secretariat 1430 Broadway

New York, N.Y. 10018 


\section{CCITT}

1. X.400 (Red Book, 1984), Message Handling Systems: System Model-Service Elements.

2. X.401 (Red Book, 1984), Message Handlịng Systems: Basic Service Elements and Optional User Facilities.

3. X.408 (Red Book, 1984), Message Handiing Systems: Encoded Information Type Conversion Rules.

4. X.409 (Red Book, 1984), Message Handling System: Presentation Transfer Syntax and Notation.

5. X.410 (Red Book, 1984), Message Handling System: Remote Operations and Reliable Transfer Server.

6. X.411 (Red Book, 1984), Message Handling Systems: Message Transfer Layer.

7. X.420 (Red Book, 1984), Message Handling Systems: Interpersonal Messaging User Agent Layer.

8. X.430 (Red Book, 1984), Message Handling Systems: Access Protocol for Teletex Terminals.

9. X.215 (Red Book, 1984), Session Service Definition for Open Systems Interconnection for CCITT Applications.

10. X.225 (Red Book, 1984), Session Protocol Specification for Open Systems Interconnection for CCITT Applications.

11. X.400 - Series Implementor's Guide (Version 3, 1986).

The above documents may be obtained from: International Telecommunications Union, Place des Nations, CH 1211, Geneve 20 SWITZERLAND. 
1. [Edge 84] S. W. Edge, An Adaptive Timeout Algorithum for Retransmission Across a Packet Switching Network, ACM Computer Communications Review, Vo1. 14, No. 2, June 1984.

2. [Jain 85] R. Jain, Divergence of Timeout Algorithms for Packet Retransmission, Proceedings IEEE Computer Communications Conference, Phoenix March 28-29, 1986.

3. [Mill 83] D. L. Mills, Internet Delay Experiments, DARPA Network Working Group RFC 非89, December 1983. 
The work on service element definitions is limited to those that are defined as 'supported' in section 13.3 of this specification. Furthermore it is not the intent of this section to define how information should be made available or presented to a MHS user, nor is it intended to define how individual vendors should design their products. In addition, statements on conformance to a specific service element and the ailocation of error codes that are generated as a result of violations of the service should be defined in the sections on conformance and errors as part of the main product specification. The main objective is to provide clarification, where required, on the functions of a service element, and in particular what the original intent of the Recommendations were.

\section{SERVICE ELEMENTS}

The following Service Elements defined in $x .400$ have been examined and require further text to be added to their definitions to represent the proposed implementation of these service elements by the X.400 SIG.

The service element clarifications are to be taken in the context of this profile.

Service elements not referenced in this section are as defined in $X .400$.

\section{PROBE}

A PRMD need not generate probes.

If a probe is addressed to and received by a PRMD, the PRMD must respond with a Delivery Report as appropriate at the time the probe was processed.

\section{DEFERRED DEL IVERY}

In the absence of bilateral agreements to the contrary, Deferred Delivery and Deferred Delivery Cancellation are local matters (i.e., confined to the originating domain) and need not be provided.

The extension of Deferred Delivery beyond the boundaries of the initiating domain is via bilateral agreement as specified in Section 3.4.2.1 of X.411.

\section{Content Type Indication}

It is required that both an originating and recipient domain be able to support P2 content type. The ability for domains to be able to exchange content types other than $P 2$ will depend on the existence of bilateral or multi-lateral agreements. 
It is required that both an originating and recipient domain be able to support IA5 text. Support for other encoded information types, for the purposes of message transfer between domains, will depend on the existence of bilateral or multi-lateral agreements.

The use of the 'unspecified' form of encoded information type should only be used when the UMPDU content represents an SR-UAPDU or contains an autoforwarded IM-UAPDU.

The original encoded information type of a message is not meaningful unless a message is converted en route to the recipient. These agreements support only IA5 text, which should not undergo conversion. The original encoded information types should be made accessible to the recipient for upward compatibility with the use of non-IA5 text message body parts.

\section{Registered Encoded Information Types}

A UMPDU with an 'unspecified' value for Original Encoded Information Type shall be delivered to the UA.

\section{Delivery Notification}

The UAContentID may be used by the recipient of the delivery notification for correlation purposes.

\section{Disclosure of Other Recipients}

This service is not made available by originating MTAE's to UAE's, but must be supported by relaying and recipient MTAE's.

By supporting the disclosure of other recipients the message recipient $c$ an be informed of the $0 / R$ names of the other recipient(s) of the message, as defined in the $P 1$ envelope in addition the 0/R Descriptors within the P2 header.

These agreements do not support initiation of disclosure of other recipients, but the information associated with it should be made accessible to the recipient for upward compatibility with support for the initiation of this service element.

\section{Typed Body}

As defined in X.400 with the addition of the Private Body Types that are to be supported. At present there is no mechanism provided within $X .420$ that would allow you to respond to reception of an unsupported body type.

Action taken in this situation is a local matter. 
It should be considered that the recipient's UA acts on behalf of the recipient, and therefore may choose to disclose all BCC recipients to each other. Therefore it is the responsibility of the originating domain to submit two or more messages, depending on whether or not each BCC should be disclosed to each other BCC.

\section{Auto-Forwarded Indication}

A UA may choose not to forward a message that was previously auto-forwarded. In addition there is no requirement for an IPM UA that does not support non-receipt or receipt notification to respond with a non-receipt notification when a message is auto-forwarded.

\section{Primary and Copy Recipients Indication}

It is required that at least one primary recipient be specified; however, for a forwarded message this need not be present. The recipient UA should be prepared to accept no primary and copy recipients to enable future interworking with Teletex, Fax, etc.

\section{Sensitivity Indication}

A message originator should make no assumptions as to the semantic interpretation by the recipients UA regarding classifications of sensitivity. For example, a personal message may be printed on a shared printer.

\section{Reply Request Indication}

In requesting this service an originator may additionally supply a date by which the reply should be sent and a list of the intended recipients of the reply. If no such list is provided than the initiator of the reply sends the reply to the originator of the message and any recipients the reply initiator wishes to include. The replytousers and the replyBy date may be specified without any explicit reply being requested. This may be interpreted by the recipient as an implicit reply request. Note that for an auto-forwarded message an explicit or implicit reply request may not be meaningful.

\section{Body Part Encryption}

The original encoded information type indication includes the encoded information type(s) of message body parts prior to encryption by the originating domain. The ability for the recipient domain to decode an encrypted body part is a local matter. Successful use of this facility can only be guaranteed if there exists bilateral agreements to support the exchange of encrypted body parts. 


\section{Forwarded IP-message Indication}

The following use of the original encoded information type in the context of forwarded messages is clarified:

- If forwarding a private message body part the originator of the forwarded message shall set the original encoded information types in the P1 envelope to undefined for that body part.

- The encoded information types of the message being forwarded should be reflected in the new original encoded information types being generated.

- See Appendix $B$ on recommended practices for the use of the delivery information as part of Forwarded IP-message.

\section{Multipart Body}

It is the intent of multipart bodies to allow for the useful and meaningful structuring of a message that is constructed using differing body part types. For example, it is not recommended that a message made up of only IA5 text should be represented as a number of IA5 body parts, each one representing a paragraph of text. 


\section{APPENDIX B: RECOMMENDED PRACTICES}

\section{B.1 RECOMMENDED PRACTICES IN P2}

a) ORDescriptor

Vendors following the NBS/OSI Workshop guidelines shall, whenever possible, generate the ORName portion of an ORDescriptor in ALL IPM Heading fields.

b) ForwardedIPMessage BodyParts

ForwardedIPMessage BodyParts should be nested no deeper than eight. There is no restriction on the number of ForwardedIPMessage Bodyparts at any given depth.

c) DeliveryInformation

It is strongly recommended that DeliveryInformation be supplied in both forwarded and autoforwarded message body parts. DeliveryInformation is useful when a message has multiple forwarded message body parts because without it, the EncodedInformationType(s) of the component forwarded messages cannot be deduced easily. Delivery Information is useful for autoforwarded messages because the Encoded InformationType of an autoforwarded message is "unspecified" and the EncodedinformationType(s) of the message cannot be determined easily without it. Absence of the EncodedInformationType(s) makes it difficult for a UA to easily determine whether the message can be rendered.

\section{B.2 RECOMMENDED PRACTICES IN RTS}

a) The calling party Network address should be used for MTA validation rather than the mTAName and password. The calling party address validation method is preferable since the recover function (in S-CONNECT) does not allow the specification of mTAName and password.

b) In the case where S-U-ABORT indicates a temporaryProblem, re-establishment of the session should not be attempted for a "sensible" time period (typically not less than five minutes).

In instances where this delay is not required or necessary, report a localsystemproblem.

c) S-U-EXCEPTION-REPORT reason codes can be interpreted as follows:

- receiving ability jeopardized (value 1)

Possible meaning: The receiving RTS knows of an impending system shutdown.

- local ss-luser error (value 5)

Possible meaning: <for further study>

- unrecoverable procedure error (value 6)

Possible meaning: the current activity is NOT recoverable. 
- non specific error (value 0 )

Possible meaning: 〈for further study>

- sequence error (value 3): The S-ACTIVITY-RESUME request specified a minor synchronization point serial number which does not match the checkpoint data.

\section{B.3 RECOMMENDED PRACTICES WITH X.409}

The following practices are recommended for use with $\times .409$.

a. The maximum length of a primitive data element is 256 .

b. Bit Strings should be built using primitive form. The constructor form should not be used except in the case of very long Bit Strings (e.g., 63Fax or Voice).

c. All defined bits of a Bit String should be present.

- Note that, in accordance with $\times .409$, defined bits need not be present; missing bits are assumed to be zero.

- To ensure upward compatibility, Bit Strings of excess length must also be allowed; the excess bits are ignored.

d. The maximum definite length should be $(2 * \star 32)-1$. <For further study>

e. It is intended that implementations support upwardly compatible changes to X.409, as defined in Version 3 of the X.400-Series Implementor's Guide, but no guarantees will be made about initial implementations.

f. The concrete encoding of ANY must be a valid X.409 type, and can only be omitted if it is an OPTIONAL element in a SET or SEQUENCE.

\section{B.4 RECOMMENDED PRACTICES FOR ORName}

Table 13.3.8 stipulates that the StandardAttributelist must contain either PrivateDomainName or Organization Name. It is recommended that, for both originator and recipients in a private domain, the PrivateDomainName field be used. 
It is recommended that there should be a DDA to be used in addresing UAs in existing mail systems, in order to curtail the proliferation of different types of DDAs used for the same purpose. The syntax of this DDA conforms to the CCITT Pragmatic Constraints, and thus has a maximum value length of 128 octets and a type length of 8 octets, each of type Printable String. One occurrance!

This DDA has the type name "ID" (in uppercase). It contains the unique identifier of the UA used in addressing within the domain. This DDA is to be exclusively used for routing within the destination domain (i.e. once routed to that domain via the mandatory components of the Standard Attribute list); any other components of the Standard Attribute list may be provided. If they conflict delivery is not made.

The contents of the valve parameter need not be validated in the originating domain or any relaying domain, but simply transferred intact to the next IITA/domain. 


\section{APPENDIX C: RENDITION OF IA5TeXt AND T61String CHARACTERS}

\section{C.1 GENERATING AND IMAGING IA5Text}

The characters that may be used in an IA5String are the graphic characters (including Space), control characters and Delete of the IA5 character repertoire ISO 646 .

The graphic characters that may be used with a guaranteed rendition are those related with positions $2 / 0$ to $2 / 2,2 / 5$ to $3 / 15,4 / 1$ to $5 / 10,5 / 15$ and $6 / 1$ to $7 / 10$ in the basic 7 -bit code table.

The other graphic characters may be used but have no guaranteed rendition.

The control characters that may be used but have no guaranteed effect are a subset consisting of the format effectors $0 / 10$ (LF), $0 / 12$ (FF) and $0 / 13$ (CR) provided they are used in one of the following combinations:

CR LF

CR FF

$L F$.. LF to start a new line to start a new page (and line) to show empty lines (always after one of the preceding combinations).

The other control characters or the above control characters in different combinations may be used but have no guaranteed effect.

The character Delete may occur but has no guaranteed effect. The IA5String in a P2 IA5Text BodyPart represents a series of lines which may be divided into pages. Each 1 ine should contain from 0 to 80 graphic characters for guaranteed rendition. Longer lines may be arbitrarily broken for rendition. Note that $X .408$ states that for conversion from IA5Text to Teletex, the maximum line length is 77 characters.

\section{C.2 GENERATING AND IMAGING T61String}

For further study. 


\section{APPENDIX D: FTAM DOCUMENT TYPES}

Part 1: Document Types

Part 2: Constraint Sets

Part 3: Abstract Syntaxes

Part 4: Transfer Syntaxes 
Entry Number: NBS-1

Document_Type Name:

\{ISO registration-authority NBS FTAM( ) document(6) UNDEF(0)\}

Document_Descriptor Value: unstructured binary file

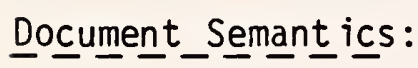

The document consists of a single data unit. The data unit consists of an unbounded sequence of data elements. Each data element is an octet string. Note: The boundaries between transferred data elements are not ma intained by the filestore.

Scope and Field of of Application:

This document type defines the contents of a file for storage and for transfer using FTAM.

Constra int Se-t_Name:

\{ISO standard 8571 constraint set name(5) unstructured(1)\}

Abstract_Syntax:

The abstract syntax of each Data Element is an instance of the ASN.1 data type OctetString.

Abstract_Syntax Name:

$\{$ ISO registration-authority NBS FTAM( ) abstract syntax(2) NBS-AS1(0)

Iransfer_syntax:

An implementation supporting this abstract syntax shall support a transfer syntax for each data element obtained by applying the ASN.1 Bas ic Encoding Rules (ISO 8825 ) to the data element and concatenating the resulting octets.

Note: This transfer syntax is not self del imiting.

Implementations may optionally support other named transfer syntaxes for this abstract syntax.

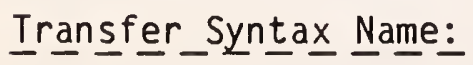

$\{$ ISO registration-authority NBS FTAM( ) transfer syntax(3) NBS-TSI(0)\} Concatenation:

Concatenation of this type with itself is possible, and produces a document of the same type consisting of one data unit which is the concatenation of the octet string(s) from one file with the octet string(s) of the other file. 
Note: The boundary of the original octet string(s) is no longer visible.

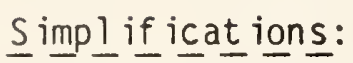

A document of this type cannot be accessed as any other document type. 
Entry Number: NBS-2

Doccument Type Name:

\{ISO registration-author ity NBS FTAM( ) document(6) VARCRLF(1)\}

Document Descriptor Value: unstructured text file

Document_Semant ics:

The document consists of a single data unit. The data un it consists of an unbounded sequence of data elements. Each data element is an IA5String. The last two characters of each data element are carriage return followed by 1 ine feed. Neither the character carriage return nor the character 1 ine feed may appear elsewhere in the data element.

Note: The boundaries between transferred data elements are not maintained by the filestore.

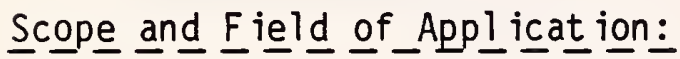

The document type defines the contents of a file for transfer using FTAM.

Note that this document type should only be used for transferring entire text files in the case where NBS-4 is not supported. It has an implic it structure which allows, for example, text files stored in UNIX format ( 1 ines terminated by $L F$ ) to be converted to a format in which 1 ines are terminated by $C R$ followed by $L F$ and vice versa.

Constra $\underline{\text { int }}$ Set_ Name:

\{ISO standard 8571 constraint set name(5) unstructured(1)\}

Abstract Syntax:

The abstract syntax of each Data Element is an instance of the ASN.I data type IA5String.

Abstract Syntax Nㅡ믈

\{ISO registration-authority NBS FTAM( ) abstract syntax(2) NBS-ASI(0)\}

Transfér_Lyntax

An implementation supporting th is abstract syntax shall support a transfer syntax (for each data element obtained by applying the ASN.1 Bas ic Encoding Rules (ISO 8825) to the data element and concatenat ing the result ing octets.

Note: This transfer syntax is not self-del imiting.

Implementations may optionally support other named transfer syntaxes for this abstract syntax. 
Iransfeㅛ_Syntax Name:

\{ISO registration-authority NBS FTAM( ) transfer syntax(3) NBS-TS1(0)\} Concatenation:

Concatenation of this type with itself is possible, and produces a document of the same type consisting of one data un it which is the concatenation of the octet string(s) from one file with the octet string(s) of the other file.

Note: The boundary of the original octet string(s) is no longer visible. Simpl if ication:

A document of this type can be accessed as a document of type NBS-1 by specifying a document type of NBS-1 in the Contents Type parameter of the F-OPEN request, and 1 imiting access context to US on F-READ. 
Entry Number: NBS-3

Document_Type aㅡe:

$\{$ ISO registration-authority NBS FTAM( ) document(6) 8859VARCRLF(2)

Document_Descriptor Value: $\quad$ unstructured text file

Doc

The document consists of a single data unit. The data un it cons ists of an unbounded sequence of data elements. Each data element is an 8859String. The last two characters of each data element are carriage return followed by 1 ine feed. Neither the character carriage return nor the character line feed may appear elsewhere in the data element.

Note: The boundaries between transferred data elements are not maintained by the filestore.

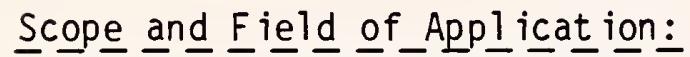

The document type defines the contents of a file for transfer using FTAM.

Note that this document type should only be used for transferring ent ire text files in the case where NBS-5 is not supported. It has an implicit structure which allows, for example, text files stored in UNIX format ( 1 ines terminated by $L F$ ) to be converted to a format in which 1 ines are terminated by $C R$ followed by $L F$ and vice versa.

Constra int Set_Name:

$\{$ ISO standard 8571 constraint set name(5) unstructured(1) $\}$

Abstract_Syntax

The abstract syntax of each data element is an instance of the data type 8859string.

Abstract Syntax $\underline{\text { Name: }}$

\{ISO registration-author ity NBS FTAM( ) abstract syntax(2) NBS-ASI(0)

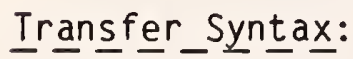

An implementation supporting this abstract syntax shall support a transfer syntax for each data element obtained by applying the ASN.1 Basic Encoding Rules (ISO 8825) to the data elements and concatenat ing the result ing octets.

Note: this transfer syntax is not self-del imiting.

Implementations may optionally support other named transfer syntaxes for th is abstract syntax. 


\section{Iransfer_Syntax aㅡe:}

\{ISO registration-authority NBS FTAM( ) transfar syntax(3) NBS-TS1(0)

\section{Concatenation:}

Concatenation of a document of this type with another document of this type is possible and produces a document of the same type cons isting of one data un it which is the concatenation of the octetstring(s) of one file with the octetstring(s) of the other file.

Note: The boundary of the original Octetstring is no longer visible.

Simplification:

A document of type NBS-3 can be accessed as a document of type NBS-1 by specifying a document type of NBS-1 in the Contents Type parameter of the F-OPENrequest, and 1 imiting access context to US on F-READ. 
Entry Number: NBS-4

Document_Type_Name:

\{ISO registration-authority NBS FTAM( ) document(6) Text(3) parameter

Note: "parameter" is a parameter which will be appended to the registered identifier in an OBJECT IDENTIFIER.

Document_Descriptor Value: Sequential Text File

Document_Semant ics:

The document consists of an unbounded series of data units. Each data un it contains one data element which is a character string. Each character is taken from the IA5 character set.

Scope and Field of of Appl icat ion:

The document type defines the contents of a file for storage and for transfer us ing FTAM.

Note: storage refers to apparent storage with in the virtual filestore. Constra int_Set Name:

\{ISO standard 8571 constra int set name(5) sequential flat(2)\}

Add it ional Constraints:

FADU Identity will be limited to begin, end, first and next. Abstract Syntax:

The abstract syntax of each data element is an instance of the data type IA5String.

The abstract syntax of each data un it is specified by the parameter. Abstract_Syntax Nㅡㄹㅡ:

\{ISO registration-authority NBS FTAM( ) abstract syntax(2) NBS-AS1(0) $\}$

Transfer_syntax:

An implementation supporting this abstract syntax shall support a transfer syntax for each data element obtained by applying the ASN.1 Bas ic Encoding Rules (ISO 8825) to the data elements and concatenat ing the resulting octets.

Note: This transfer syntax is not self-del imiting.

Implementations may optionally support other named transfer syntaxes for this abstract syntax.

Iransfer Syntax Name:

\{IS0 registration-authority NBS FTAM( ) transfer syntax(3) NBS-TS1(0) 


\section{Concatenation:}

Concatenation of a document of this type with another document of this type is possible and produces a document of the same type consisting of a series of IASStrings which is the result of placing the series of IA5Strings from one file of this type after the last IASString in the original file.

Note: The boundary of the original sequence is no longer vis ible.

Simpl if icat.ion:

A document of type NBS -4 can be accessed as a document of type NBS-1 by specifying a document type of NBS-1 in the Contents Type parameter on the F-OPENrequest, and 1 imiting access context to UA on F-READ. 
Entry Number: NBS-5

Document_Type Name:

\{ISO registration-authority NBS FTAM( ) document(6) 8859Text(4) parameter

Note: "parameter" is a parameter which will be appended to the registered identifier in an object Identifier.

Document_Descriptor Value: Sequential Text File

Doc

The document consists of an unbounded series of data un its. Each data un it contains one data element which is a character string. Each character is taken from the ISO $8859 / 1$ character set.

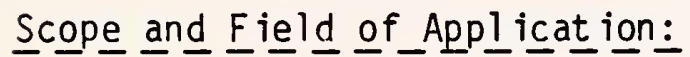

The document type defines the contents of a file for storage and transfer us ing FTAM.

Note: Storage refers to apparent storage with in the virtual filestore.

Constra int $\underline{\text { Set }}$ Name:

\{ISO standard 8571 constraint set name(5) sequential flat(2)

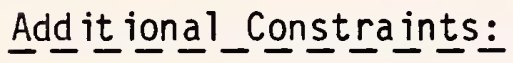

FADU Identity will be limited to begin, end, first and next.

Abstract Syntax

The abstract syntax of each data element is an instance of the data type 8859string.

The abstract syntax of each data un it is specified by the parameter.

Abstract Syntax №me:

\{ISO registration-authority NBS FTAM( ) abstract syntax(2) NBS-ASI(0)\}

Transfer Syntax

An implementation supporting this abstract syntax shall support a transfer syntax for each data element obtained by applying the ASN.1 Bas ic Encoding Rules (ISO 8825) to the data elements and concatenating the resulting octets.

Note: This transfer syntax is not self-delimiting.

Implementations may optionally support other named transfer syntaxes for this abstract syntax. 


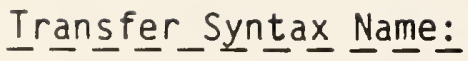

\{ISO registration-authority NBS FTAM( ) transfer syntax(3) NBS-TS1(0)\}

\section{Concatenation:}

Concatenation of a document of this type with another document of this type is possible and produces a document of the same type consisting of a series of 8859strings which is the result of placing the series of 8859strings from one file immediately following the last 8859String in the original file.

Note: The boundary of the original series is no longer visible.

Simpl if ication:

A document of type NBS-5 can be accessed as a document of type NBS-1 by specifying a document type of NBS-1 in the Contents Type parameter on the F-OPENrequest, and 1 imiting access context to UA on F-READ. 
Entry Number: NBS-6

Document Type Nㅡ를

\{ISO registration-authority NBS FTAM( ) document(6) SEQUENTIAL(5) parameter

Note - "parameter" is a parameter which will be appended to the registered ident if ier in an Object Ident if ier.

Doçument_Descriptor Value: $\quad$ Sequential File

Doçument_Semantiç

The document cons ists of an unbounded series of data un its. Each data un it contains an unbounded series of data elements. Each data element is a data type from the set of primitive data types defined in the main body of this document. Each data un it contains the same data element types in the same order as all other data un its.

Scope and Field of Appl ic aㅡ ion:

The document type defines the contents of a file for storage and transfer us ing FTAM.

Note: Storage refers to apparent storage with in the virtual filestore.

Constra int $\underline{\text { Set }}$ Name:

\{ISO standard 8571 constraint set name(5) sequential flat(2)\}

Add it ional Constraints:

FADU Identity will be 1 imited to begin, end, first, and next.

Abstract_syntax

The abstract syntax of each data element is an instance of one of the primit ive data types defined in the ma in body of this document.

The Abstract syntax of each data un it is specified by the parameter.

Abstract Syntax №me:

\{ISO registration-authority NBS FTAM( ) abstract syntax(2) NBS-ASI(0)\} opt ionally, \{ISO registration-authority NBS FTAM( ) abstract syntax(2) NBS-AS2 (1) \}

Irans $\underline{\text { fer }}$ Syntax

An implementation supporting this abstract syntax shall support a transfer syntax for each data element obtained by applying the ASN.1 Bas ic Encoding Rules (ISO 8825) to the data elements and concatenating the resulting octets.

Note: This transfer syntax is not self-delimiting. 
Implementations may optionally support other named transfer syntaxes for this abstract syntax.

Irans $\underline{\text { eq }}$ _Syntax $\underline{\text { Name: }}$

\{ISO registration-authority NBS FTAM( ) transfer syntax(3) NBS-TS1(0)\} Concatenation:

Concatenation of a document of this type with another document of this type is possible and produces a document of the same type consisting of a series of data un its which is the result of placing the series of data units from one file immediately following the last data unit of the or iginal file.

Note: The boundary of the original file is no longer visible.

Simplification:

A document of type NBS- 6 can be accessed as a document of type NBS-1 by specifying a document type of NBS-1 in the Contents Type parameter in the F-OPENrequest, and $l$ imiting access context to UA on F-READ. 
Entry Number: NBS-7

Document_Type Name:

\{ISO registration-authority NBS FTAM ( ) document(6) RANDOM(6) parameter

Note: "parameter" is a parameter which will be appended to the registered identifier in an object Ident if ier.

Document_Descriptor Value: Random Access File

Document_Semantics:

The document consists of an unbounded series of data units. Each data un it contains an unbounded series of data elements. Each data element is a data type from the set of primitive data types defined in the ma in body of th is document. Each data unit contains the same data types in the same order as all other data un its in the file.

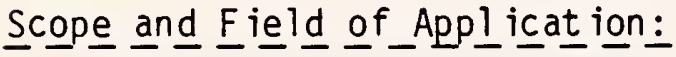

The document type defines the contents of a file for storage and transfer us ing FTAM.

Note: storage refers to apparent storage with in the virtual filestore.

Constra int_Set_name:

\{ISO registration-authority NBS FTAM( ) constraint set name(5) NBS Ordered Flat(2)\}

Abstract_Syntax:

The abstract syntax of each data element is an instance of one of the primitive data types defined in the ma in body of this document.

The Abstract syntax of each data un it is specif ied by the parameter.

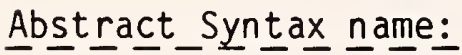

\{ISO registration-authority NBS FTAM( ) abstract syntax(2) NBS-AS1(0)\}

optionally, \{ISO registration-authority NBS FTAM( ) abstract syntax(2) NBS-AS2(1)\}

Iransfe﹎.Syntax

An implementation supporting this abstract syntax shall support a transfer syntax for each data element obtained by applying the ASN.1 Bas ic Encoding Rules (ISO 8825) to the data element and concatenating the resulting octets.

Note: This transfer syntax is not self del im it ing.

Implementations may optionally support other named transfer syntaxes for th is abstract syntax. 
Trans fer Syntax №me:

\{ISO registration-authority NBS FTAM( ) transfer syntax(3) NBS-TS1(0)

Concatenation:

Concatenation of a document of this type with another document of this type is possible and produces a document of the same type cons isting of a series of data units which is the result of placing the series of data units from one file immediately following the last data un it of the original file.

Note: the boundary of the original file is no longer visible.

Simpl if ication:

A document of type NBS-7 can be accessed as a document of type NBS-1 by specifying a document type of NBS-1 in the Contents Type parameter of the F-OPENrequest, and 1 imiting access context to UA on F-READ.

A document of type NBS-7 can be accessed as a document of type NBS- 6 by specifying a document type of NBS- 6 in the Contents Type parameter of the F-OPENrequest. 
Entry Number: NBS-8

Document Type Name:

\{ISO registration-authority NBS FTAM( ) document(6) INDEXED(7) p1 p2 $\}$

Note: "p1" and "p2" are parameters which will be appended to the registered ident if ier in an object Identifier.

Document_Descriptor Value: Indexed Sequential File

Document_Semantics:

The document consists of an unbounded series of data un its. Each data un it is an unbounded series of data elements. Each data element is a data type from the set of primitive data types defined in the ma in body of this document. Each data un it contains the same data types in the same order as all other data units in the file.

Each data un it in the file has a key associated with it. The key of each data unit is of the same data type as the key of all other data units in the file and is a single data element from the set of primitive data types defined in the ma in body of this document.

Scope and Field of Appl ication:

The document type defines the contents of a file for storage and for transfer using FTAM.

Constra int Se-t_Name:

$\{$ ISO registration-authority NBS FTAM( ) constraint set name(2) Indexed Flat $(1)\}$

Abstract_Syntax

The abstract syntax of each data element is an instance of one of the primitive data types defined in the main body of this document.

The Abstract syntax of each data unit is specified by the parameter pl.

The Abstract syntax of the data un it key (FADU Identifier) is specified by the parameter p2.

Abstract_Syntax Nㅡ므를

$\{$ ISO registration-authority NBS FTAM( ) abstract syntax(2) NBS-AS1(0) optionally, \{ISO registration-authority NBS FTAM( ) abstract syntax (2) NBS-AS2(1)\} 


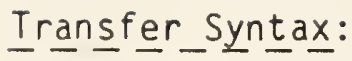

An implementation supporting th is abstract syntax shall support a transfer syntax for each data element obtained by applying the ASN.1 Bas ic Encoding Rules (ISO 8825) to the data elements and concatenating the resulting octets.

Note: this transfer syntax is not self-del imiting.

Implementations may optionally support other named transfer syntaxes for this abstract syntax.

Iransfer_Syntax Name:

\{ISO registration-authority NBS FTAM( ) transfer syntax(3) NBS-TS1(0) Concatenation:

A document of this type may not be concatenated with a document of this type or any other type.

Simpl if ication:

A document of type NBS-8 can be accessed as a document of type NBS-1 by spec ifying document type NBS-1 in the Contents Type parameter on the F-OPENrequest, and 1 imiting access context to UA on F-READ.

A document of type NBS-8 can be accessed as a document of type NBS- 6 by specifying document type NBS-6 in the Contents Type parameter on the F-OPENrequest.

A document of type NBS-8 can be accessed as a document of type NBS-7 by specifying document type NBS-7 in the Contents Type parameter on the F-OPENrequest. 
Entry Number: NBS-9

Document Type Name:

\{ISO registration-authority NBS FTAM( ) document(6) FILE_DIRECTORY(8)

Document Descriptor aㅣuㅡ: Filedirectory File

Doçument_Semant ics:

The document consists of an unbounded sequence of data un its. Each data un it consists of one and only one data element of type FileDirectoryEntry (a complex data type defined in the ma in body of this document).

Scope and Field of of Appl ication:

This document defines the contents of a file for transfer (not for storage) us ing FTAM.

Constra $\underline{\text { Cont }}$ Set_Name:

\{ISO registration-authority NBS FTAM( ) constraint set name(5) Sequential Flat (1) $\}$

Add it ional Constraints:

FileDirectory Files may be Selected, Opened, Read, Closed, Created, and Deleted. They may not be Written or Mod if ied (except as a side-effect of actions performed on individual files contained with in a Filedirectory). DataUnits within a FileDirectory may only be accessed sequentially.

Abstract Syntax:

An indefinite series of data un its. Each data un it contains one data element of type FileDirectoryEntry. Each data element cons ists of a required $F$ ileName and a number of optional Attributes.

Abstract_Syntax $\underline{\text { S }}$ Name:

\{ISO registration-authority NBS FTAM( ) abstract syntax(3) filedirectory entry $(0)\}$

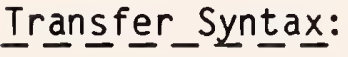

An implementation supporting this data type shall support a transfer syntax for each data value obta ined by applying ASN.1 Basic Encoding Rules to the data type FileDirectoryEntry in the data value and concatenating the result ing octets.

Note: this transfer syntax is not self-delimiting.

Implementations may also support other named transfer syntaxes for this abstract syntax. 


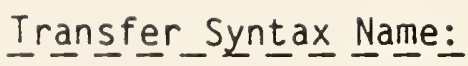

\{ISO registration-authority NBS FTAM( ) transfer syntax(3) NBS-TS1(0) Concatenation:

A document of this type cannot be concatenated with a document of this type or any other type.

Simpl if içation:

A document of this type cannot be simplified. 
Part 2: Constraint Sets

Constaint Set Title: NBS-Ordered Flat

Constraint Set Name:

\{ISO registration-authority NBS FTAM( ) constraint set name(5)

NBS Ordered Flat(2)

Field of Application:

Node Names:

Act ions:

Special Act ion Parameters:

Special Act ion Semantics:
Files which are structured into a sequence of individual FADUs and to which access may be made on a FADU bas is by position in the sequence.

none

Locate, Read, Replace, Insert, Erase

none

Erase: Used on the root node to empty the file. When used on a leaf node, it leaves a FADU with no associated data unit.

Insert: Allowed only at end of file. The new node is inserted following all existing nodes in the file or on a leaf node with no existing data unit. The inserted data un it is associated with the currently existing leaf node.

Available Access Contexts: HA, FA, UA, US

Erase and Locate Context: HA

Constraints on Structure: The root node shall not have an associated data un it. All children of the root node shall be leaf nodes and may have an associated data unit. All arcs from the root node shall be of length one.

Creation State:

Root node without an associated data unit.

FADU Ident ity:

begin, end, first, last, current, next, previous, traversal number (greater than or equal to one)

Location After Open:

root node

Beginn ing of File:

root node

End of File:

No node is selected. Previous gives the last node in the traversal sequence, current and next result in an error. 
Constraint Set Title: Indexed Flat

Constraint Set Name:

\{ISO registration-authority NBS FTAM( ) constraint set name(5)

NBS Indexed Flat(1)\}

Field of Application:

Node Names:

Actions:

Special Action Parameters:

Special Action Semantics:

Available Access Contexts:

Erase and Locate Context:
This constraint set is for representing single key ISAM files where the keys are the FADU identifiers for the leaf nodes. The keys are restricted to be ing single primitive data types, and restricted to all keys being of the same primitive data type.

Any single primitive data type.

Locate, Read, Replace, Insert, Erase

none

Locate: The specif ied FADU is made the current FADU. If the FADU Id form is used, the least recently inserted FADU with the specified FADU at level 1 is located.

Read: Allowed at root and leaves. If there is another FADU after (in pre-order traversal order) the one read with the same FADU Id, a diagnostic on TRANSFER_END will indicate this fact.

Insert: Insert the specified FADU (level 1 only) in the lexical order of the key primitive data type. If there is al ready another FADU with the specified FADU Id, insert the new one after (in pre-order traversal) the existing FADUs and indicate that this was done via a diagnostic on TRANSFER END.

Replace: Allowed only at leaves and only in access context US (DU only w/o del imiters). Only allowed with write operation of "current" (i.e., preceded by locate) or "Previous" (i.e., preceded by read).

Erase: If the addressed FADU is the root, the file is reduced to the in itial state.

$H A, F A, U A$, US

$H A$ 
Constraints on Structure: The root node shall not have an associated data un it or/and FADU Id. All children of the root node shall be leaf nodes and shall have an associated data unit and FADU Id. All arcs from the root node shall be of length one. Some primit ive types may not be supported as keys.

Creation State:

Root node without an associated data un it or FADU Id.

FADU Ident ity:

beg in, end,

current, next, previous,

FADU Id

Location After Open:

root node

Beginning of File:

End of File:

root node

No node is selected. Previous gives the last node in the traversal sequence, current and next result in an error. 
Part 3: Abstract Syntaxes

Abstract Syntax: NBS-AS1

Abstract Syntax Name:

\{ISO registration-authority NBS FTAM( ) Abstract Syntax(2) Bas ic(0)\} Abstract Syntax Defin ition:

DE ::=Cho ice $\{$ INTEGER, BOOLEAN, IASString, 8859String, OCTETSTRING, Un iversalTime, General izedT ime, Nul1\}

8859String ::= [PRIVATE 1] Impl ic it 8859CharacterString

Transfer syntax name: - - 8859CharacterString is a string of characters from -- the ISO 8859 character set

\{ISO registration-authority NBS FTAM( ) Transfer Syntax(4) NBS-TS1 (0)\}

Abstract Syntax: NBS-AS2

Abstract Syntax Name:

\{ISO registration-authority NBS FTAM( ) abstract syntax(2) FloatingPoint(1)\} Abstract Syntax Def in ition:

Float ingPo int Number ::= [PRIVATE O] CHOICE

$\{$ fin ite [0] IMPLICIT SEQUENCE

\{

$$
\text { Sign, }
$$
mant issa BITSTRING, \} exponent INTEGER

inf in ity [1] IMPLICIT Sign, signall ing $\mathrm{NaN}$ [2] Impl ic it $\mathrm{NaN}$, quiet NaN [3] IMPLICIT NaN,

Sign $::=$ zero [4] IMPL ICIT NULL

$\mathrm{NaN}::=$ INTEGER

Transfer Syntax Name:

\{ISO registration-authority NBS FTAM( ) transfer syntax(3) NBS-TS1(0) 
Abstract Syntax: NBS-AS3

Abstract_Syntax Name:

\{ISO registration-authority NBS FTAM( ) abstract syntax(2) FileDirectoryElement(2)\}

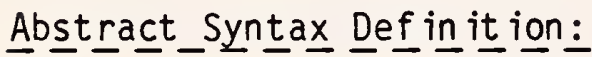

FileDirectoryEntry : := [PRIVATE 2] IMPLICIT SEQUENCE \{ fileName Graphicstring, ContentsType $\}$

ContentsType ::= CHOICE document-type-Name[0] IMPLICIT OBJECT IDENTIFIER, constra int-set-and-abstract-synt ax [1] IMPL ICIT SEQUENCE constra int-set-Name[0] IMPLICIT OBJECT IDENTIFIER, abstract-syntax-Name[1] IMPLICIT OBJECT IDENTIFIER $\}$

Iransfer_Syntax №me:

\{ISO registration-authority NBS FTAM( ) transfer syntax(3) NBS-TS1(0) 
Part 4: Transfer Syntaxes

Transfer Syntax: NBS-TS1

Transfer Syntax Name:

\{ISO registration-authority NBS FTAM( ) transfer syntax(3) NBS-TSI(0)\}

Encoding Rules:

ASN.1 Basic Encoding Rules shall apply

The first bit of a "fraction" must be " 1 "

Transfer Syntax Def in ition:

The transfer syntax shall be that which results from applying the encoding rules described above to the individual data elements.

\section{ADDENDUM 1}

Note on FTAM and X.400 Character Sets

On July 21, 1986 a group of twelve individuals from the FTAM and X.400 SIGs met to resolve differences in recommended use of character sets. The following was agreed (in favor, 9; opposed, 2; abstaining, 1) by these individuals:

"Both SIGs should implement IA5 for the current phase of development, and independently support expanded character sets. It is recommended that the FTAM and X.400 SIGs support both $8859 / 1$ and $6937 / 2$ in the next phase of the ir agreements."

Neither SIG brought forward to the plenary on Thursiay July 24, 1986 a recommendation on this issue. However, it was raised for plenary discuss ion. The plenary felt ( in favor, 22; opposed, 3; abstain ing, 1) that th is information should be carried in some form in this document in addition to inclusion in the minutes. Hence, it is included as an addendum so as to keep the information associated with this document while showing that it has not been accepted for inclusion in the ma in body of this document. 
You will receive the documents from the next workshop by either attending the workshop or completing and returning the form below.

\section{READER RESPONSE FORM}

Please retain my name for the next mailing of the NBS/OSI Implementors Workshop

NAME

ADDRESS

PHONE NO.

Mail this page to: Kim Brink

National Bureau of Standards

B1dg. 225/B217

Gaithersburg, MD 20899 
BIBLIOGRAPHIC DATA

SHEET (See instructions)

4. TITLE AND SUBTITLE

Implementation Agreements Among Implementors of OSI Protocols

5. AUTHOR(S)

John Heafner, Editor

6. PERFORMING ORGANIZATION (If joint or other th on NBS, see in structions)

NATIONAL BUREAU OF STANOAROS

DEPARTMENT OF COMMERCE

. Contract Grant No.

Gaithersburg, MD 20899

9. SPONSORING ORGANIZATION NAME AND COMPLETE ADDRESS (Street, City, Stote, ZIP)

10. SUPPLEMENTARY NOTES

Document describes a computer program; SF-185, FIPS Software Summary, is attached.

11. ABSTRACT (A 200-word or less foctual summary of most significant information. If document includes a significant bibliography or literoture survey. mention it here)

This document records current agreements on implementation details of Open Systems Interconnection protocols among the organizations participating in the NBS/OSI Workshop Series for Implementors of OSI Protocols. These decisions are documented to facilitate organizations in their understanding of the status of agreements. This is a standing document that is updated after each workshop (about every 2-1/2 months). A reference list of standards and a list of contributing organizations are included in the Appendix.

12. KEY WORDS (Six to twelve entries; alphabetical order; capitalize only proper names; and separate key words by semicolons)

local area networks; NBS/OSI Workshop; network protocols; Open systems interconnection; OSINET; testing protocols

\section{AVAILABILITY}

Unlimited

X For Official Distribution. Do Not Release to NTIS

Order From Superintendent of Documents, U.S. Government Printing Office, Washington, D.C. 20402.

Order From National Technical Information Service (NTIS), Springfield, VA. 2216I
14. NO. OF

PRINTED PAGES

15. Price 

